NoTKE:

"Thts nepot was prepanod as an 2ccousas af wask sponvorer by

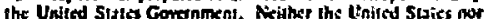

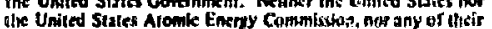
lin Uniled Staks Aromk Enaty Commiston, noh any of litif

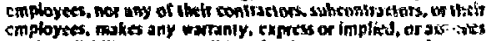

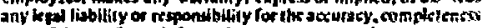

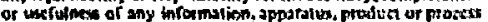

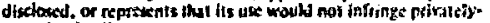
owned righte."

Printed in the United States of America Available from

National Technical Information Service

U.S. Department of Commerce 5285 Port Royal Road Springfield, Virginia 22151

Price: Printed Copy $\$$; Microfiche 80.95

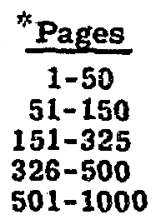

NT)

Selling Price

$\$ 4.00$

$\$ 5.45$

$\$ 7.60$

$\$ 10.60$

$\$ .13 .60$ 
T1D-4500, UC-32

Mathematics and Computers

\section{㢟 \\ LAWRENCE LNEFMOAE LABORATOAY \\ universifyol Ceftomis, Ltermoro, Cestomia 84560}

\section{UCRL-51426 \\ PABSOT: A FORTRAN CODE THAT PRODUCES GRAPHICAL REPRESENTATIONS OF NUCLEAR BLACKOUT EFFECTS ALONG A REENTPY TRAJECTORY}

M. D. Maples

MS. date: August 17, 1975

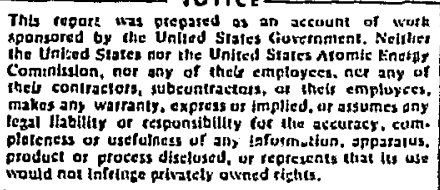




\section{Contents}

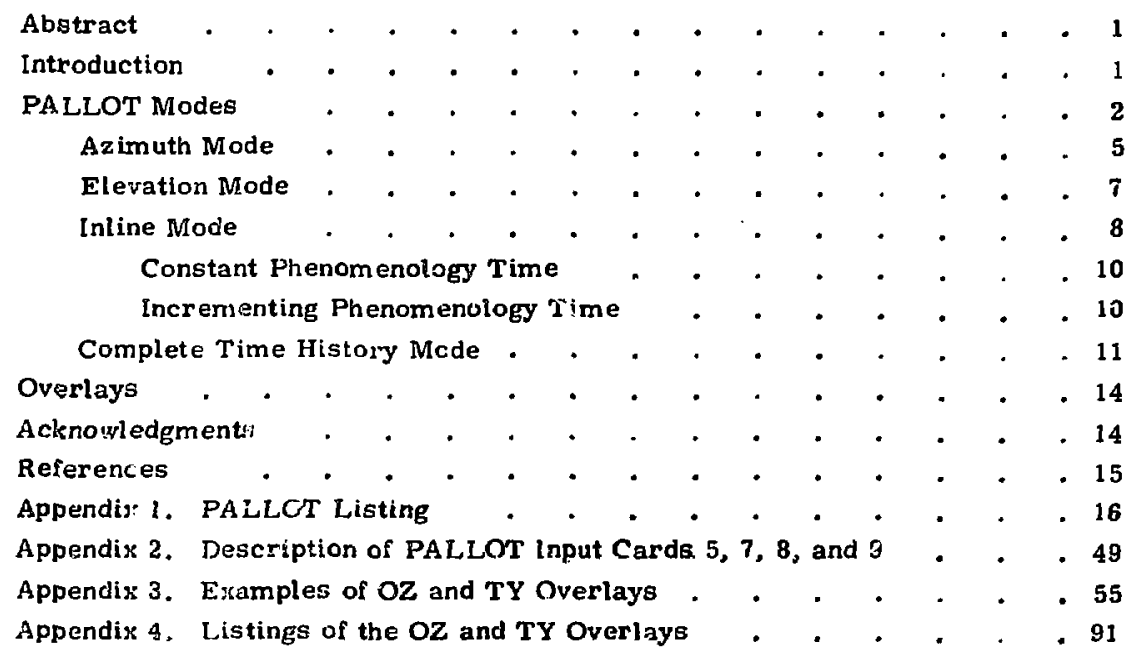




\title{
FALLOT: A FORTRAN CODE THAT PRODUCES GRAPHICAL REPRESENTATIONS OF NUCLEAR BLACKOUT EFFECTS ALONG A REENTRY TRAJECTORY
}

\begin{abstract}
Âbstract
The PALLOT code was developed to simplify th $\subseteq$ assimilation of data relating to effects of nuclear blackout on particular reentry trajectories. This code operates in three inodes: azimuth, elevation, and inline. In the azimuth and elevation modes, the PALLOT picture represents a farnily of trajectories that only vary, respectivel?, in approach azimuth and

impact el evation angle. The inline mode depicts a set of reentry vehicles approashing on the same trajectory through a nuclear environment. The code, writien in Fortran IV, was developed on the $C D C 7600$ computer; it will run on the CDC 6600 without modification although the run times may be excessively loing.
\end{abstract}

\section{Introduction}

PALLOT ${ }^{i, 2}$ (PONDER ALong Locus Or Trajectories) is an extensior. of the code PONDER ${ }^{3-6}$ (Pictures Of Nuclear Device Effects From RANC), which provides a convenient rueans of displaying data from the RANC ${ }^{\top}$ (Radar Absorption Noise and Clutter) code. The PALLOT code is documented in this report.

The PALLOT code relates blackout absorption and refraction to system, geometric, and weapon parameters. PALLOT provides pictorial representations of the time-dynamic effects of blackout on a radar bearn; it uses the RANC code for blackout calculations. The spatial extent and degree of blackout can be determined by systemati zally selecting a number of target sample points on a prescribed surfane.
PALLOT has four modes of operation: the azimuth, elevation, inline, and complete time history modes, In the first two modes the PALLOT code zenerates a surface of sample points described by a set of trajectories, with common impact points distributed in azimuth and eleration, respectively. Time can be ailowed to advance for the burst phenomenolngy as one samples each line on this surface. The rate of time advance is determined by the reentry vehicle ( $\mathrm{RV}$ ) parameters chosen. Thus, the time dynamic blackout for a number of RV trajectories can be observed on one picture. In contrast, the inline mode provides a picture of the blackout histo $y$ along a trajectory as a function of phenomenology time. In this case a number of RV's can be observed moving 
along the same trajectory. The complete time history mode holds a set of RV's stationary along a selected trajectory while phenomenology time is allowed to increment. This mode allows the accumulation of the total phenomenology effects on a selected trajectory and encompasses the information produced in the inline mode. The tradeoff between these two modes is between their associated run times (the complete time history mode runs five to six times longer than the equivalent inline mode) and their respective information content.

Pictures can be obtained for signal/ noise, signal/noise-plus-clutter, signal/ clutter, and clutter/noise ratios. The cause of the blackout le.g., fireball ioni- zation, $\gamma$-ray ionization, etc.) is identified on a picture. The area blacked out on the surface is calculated and can be used for doing trend analysis and parameter sensitivity studies, PALLOT is also useful as a visual aid in understanding the RANC phenomenology.

The remainder of this report is divided into three sections. In the following section the four modes of the PALLOT code are covered in detail. The next section consists of a brief explanation of the overlays presenily being used at the Lawrence Liventmore Laboratory. The last section contains the appendices, which include program listings, descriptions of input variables, and sample overlays.

\section{PALLOT Modes}

PALLO' utilizes the RANC IVA ${ }^{7}$ package developed by GE-TEMPO. The block diagram in Fig. 1 shows the relationship between PALIOT and RANC IVA.

Figure 2 lists the general subroutine flow in PALLOT. An asterisk denotes a subroutine written or modified (from RANCI for PALLOT: all other subroutines are RANC IVA subroutines. Refer to Appendix 1 for subroutine listings.

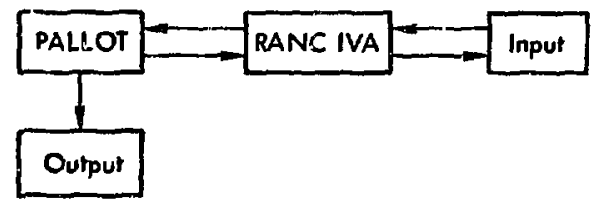

Fig. 1. Block diagram of the rolationship between PALLOT and RANC IVA.
Figure 3 shows RANC IVA's unmodified input format, while Fig. 4 shows PAJLOT's

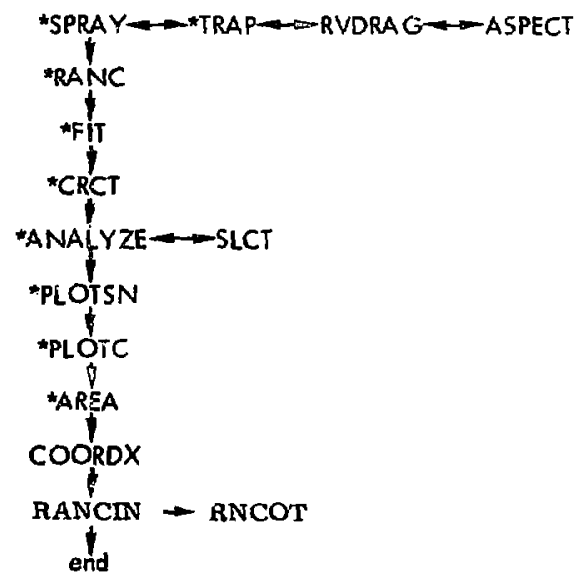

Fig. 2. Subroutine flow in PALLC" The asterisks denote sub: outined written or modified for PALLOT. 


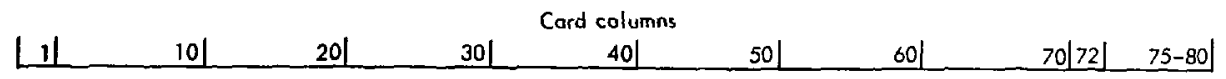

\begin{tabular}{|c|c|c|c|c|c|c|c|c|c|}
\hline & $\begin{array}{c}\text { Radar } \\
\text { requency } \\
(\mathrm{MHz})\end{array}$ & $\begin{array}{c}\text { Peak } \\
\text { power } \\
(\mathrm{MW})\end{array}$ & $\begin{array}{c}\text { Range } \\
\text { resolution } \\
(\mathrm{m})\end{array}$ & $\begin{array}{c}\text { Velocity } \\
\text { resolution } \\
(\mathrm{m} / \mathrm{sec})\end{array}$ & $\begin{array}{c}\text { Transmitter } \\
\text { ontenno } \\
\text { gain }(\mathrm{dB})\end{array}$ & $\begin{array}{c}3 \mathrm{~dB} \\
\text { beamwidth } \\
(\mathrm{deg})\end{array}$ & $\begin{array}{c}\text { Sidelobe } \\
\text { level } \\
(\mathrm{dB} \text { down })\end{array}$ & $\begin{array}{c}\mathrm{k}_{\mathrm{i}} \\
\mathrm{i}\end{array}$ & $\begin{array}{c}\text { Optional } \\
\text { alpha } \\
\text { lobel }\end{array}$ \\
\hline
\end{tabular}

\begin{tabular}{|c|c|c|c|c|c|c|c|c|c|}
\hline 2 & $\begin{array}{c}\text { Minimum } \\
\mathrm{S} / \mathrm{N} \\
(\mathrm{b})\end{array}$ & $\begin{array}{c}\text { Sys noise } \\
\text { temperature } \\
(\mathrm{OK})\end{array}$ & $\begin{array}{c}\text { Receiver } \\
\text { noise bnwth } \\
(\mathrm{kHz})\end{array}$ & $\begin{array}{c}\text { Pulse } \\
\text { compression } \\
\text { rotio }\end{array}$ & $\begin{array}{c}\text { Receiver } \\
\text { antenno } \\
\text { gain (dB) }\end{array}$ & $\begin{array}{c}\text { 3 dB } \\
\text { beamwidth } \\
(\mathrm{deg})\end{array}$ & $\begin{array}{c}\text { Sidelobe } \\
\text { level } \\
(\mathrm{dB} \text { down })\end{array}$ & $\begin{array}{c}\text { Optional } \\
\text { alpho } \\
\text { label }\end{array}$ \\
\hline
\end{tabular}

\begin{tabular}{|c|c|c|c|c|c|c|c|}
\hline 3 & $\begin{array}{l}\text { Anienno } \\
\text { sidelobe } \\
\text { spec }\end{array}$ & $\begin{array}{c}\text { Arroy scon } \\
\text { limit } \\
\text { (deg) }\end{array}$ & $\begin{array}{l}\text { Arroy foce } \\
\text { elevation } \\
\text { (deg) }\end{array}$ & $\begin{array}{l}\text { First foce } \\
\text { azimuth } \\
\text { (deg) }\end{array}$ & $\begin{array}{l}\text { Second foce } \\
\text { azimuth } \\
\text { (deg) }\end{array}$ & $\begin{array}{l}\text { Third face } \\
\text { azimuth } \\
\text { (deg) }\end{array}$ & $\begin{array}{l}\text { Fourth face } \\
\text { ozimuth } \\
\text { (deg) }\end{array}$ \\
\hline
\end{tabular}

\begin{tabular}{|c|c|c|c|c|c|c|c|c|}
\hline 4 & $\begin{array}{c}\text { N latít. de } \\
\text { of origin } \\
\text { (deg) }\end{array}$ & $\begin{array}{c}\text { E longitude } \\
\text { of origin } \\
\text { (deg) }\end{array}$ & $\begin{array}{c}\text { Day or night } \\
(1 \text { or } 0)\end{array}$ & $\begin{array}{c}\text { Calculation } \\
\text { aption }\end{array}$ & $\begin{array}{c}\text { Normol } \\
\text { outpus } \\
\text { option }\end{array}$ & $\begin{array}{c}\text { Beam- } \\
\text { pointing } \\
\text { optian }\end{array}$ & $\begin{array}{c}\text { Detoiled } \\
\text { autput } \\
\text { option }\end{array}$ & \\
\hline
\end{tabular}

Rejar location

\begin{tabular}{|c|c|c|c|c|c|c|c|c|c|}
\hline 5 & $Y(N)$ & $Z(N)$ & $\begin{array}{c}\text { Magnetic dip } \\
\text { at rodor } \\
\text { (deg; }\end{array}$ & $\begin{array}{c}\text { Magnetic } \\
\text { voriation at } \\
\text { radar (deg) }\end{array}$ & $\begin{array}{c}\text { Ground } \\
\text { range }\end{array}$ & Azimuth & $\begin{array}{c}\text { Optional } \\
\text { alpho } \\
\text { label }\end{array}$ \\
\hline
\end{tabular}

Burst location (up to 50 bursts)

\begin{tabular}{|c|c|c|c|c|c|c|c|c|c|}
\hline 6 & $X(N)$ & $Y(N)$ & $Z(N)$ & $\begin{array}{c}\text { Detonation } \\
\text { rime } \\
(\mathrm{sec})\end{array}$ & $\begin{array}{c}\text { Tolol } \\
\text { yield } \\
(\mathrm{Mt})\end{array}$ & $\begin{array}{c}\text { Fission } \\
\text { fraction }\end{array}$ & $\begin{array}{c}\text { Device } \\
\text { type }\end{array}$ & $\begin{array}{c}\text { Optional } \\
\text { alpho } \\
\text { lobel }\end{array}$ \\
\hline
\end{tabular}

\begin{tabular}{|l|l|l|l|l|l|l|l|l|l|}
\hline 7 TSEC(NT) & NAXNMX & NCRCT & $\begin{array}{l}\text { SIGMA } \\
\text { (RCS) }\end{array}$ & MROTE & QREF & DLTH & $\begin{array}{c}\text { Optional } \\
\text { Glpho } \\
\text { Llabel } \\
\text { label }\end{array}$ \\
\hline
\end{tabular}

\begin{tabular}{|l|l|l|l|l|l|l|l|l|l|}
\hline 8 & atifo & GRANGE & VFPEO & VVEL & hreF & Trefo & Beta & $\begin{array}{c}\text { M Optional } \\
\text { A } \\
\text { D }\end{array}$ \\
alpho \\
label
\end{tabular}

\begin{tabular}{|c|c|c|c|c|c|c|c|c|c|}
\hline PLDEN & RDEN & UNITHO & UNITVO & NMXO & NPXO & NHR NHRI & \\
\hline
\end{tabular}

\begin{tabular}{|l|l|l|l|l|l|l|l|l|l|}
\hline 9A & SBOCRIT & ISSNBOCR & THCLTR & DTHCLTR & THDIFF & DTHDIFF & & & \\
\hline
\end{tabular}

Blank card at end of each cose

Fig. 4. PALLOT input format. 


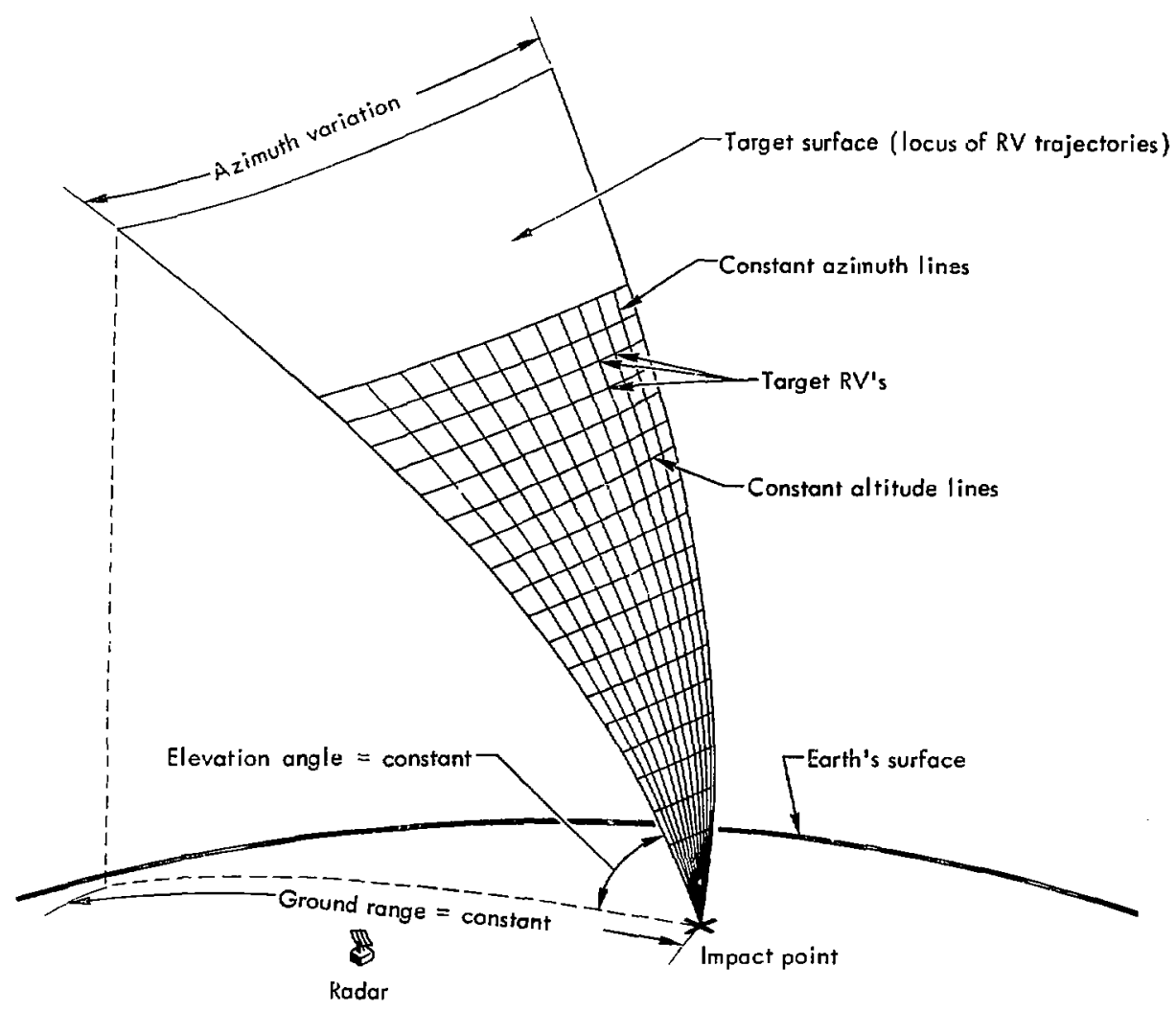

Fig. 5. PALLOT azimuth mode.

\section{AZIMUTH MODE}

When IFL ${ }^{\text {;* }}$ is set equal to zero, PALLOT runs in the azimuth mode. The azimuth mode keeps the trajectory ground range and el evation angle constant while varying the azimuth angle to the impact point (see Fig. 5). The azimuth mode operates in two formats: constant phenomenology time and changing

\footnotetext{
Definitions of terms may be found in Appendix 2.
}

phenomenology time. The TMADV switch in column 72 of card eight controls these two formats. When TMADV equals zero, the azimuth surface will be produced at a constant phenomenology time. When TMADV equals one, the azimuth surface will be produced with changing phenomenology time. The difference between these two formats is that in the constant phenomenology format, RANC IVA calculates the blackout only at the given TSEC (NT) time for the complete azimuth mode surface; but, when TMADV equals 


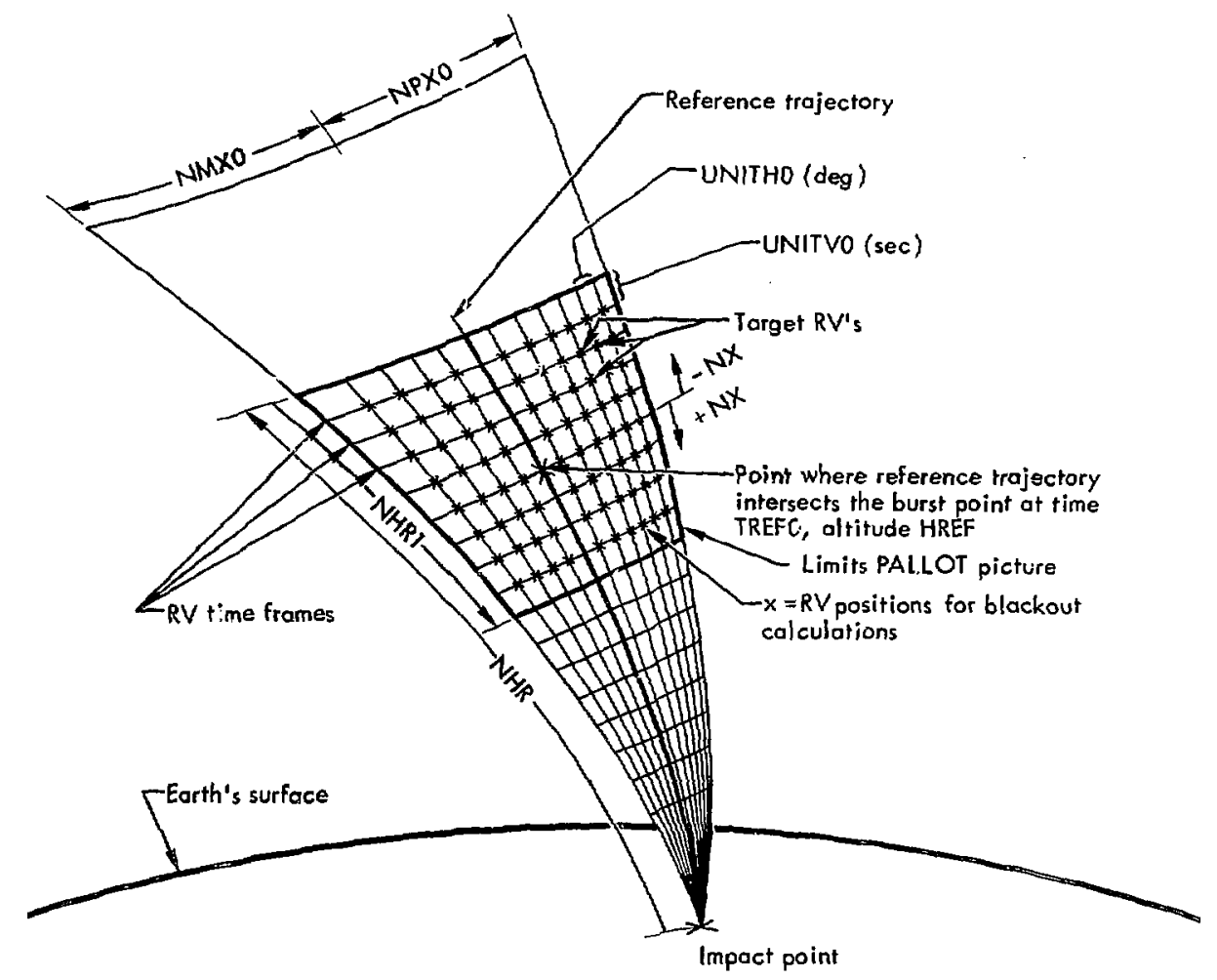

Fig. 6. Detailed view of the PALLOT azimuth mode.

Ine, RANC IVA calculates blackout relative to the burst point, reference trajecto: $y$ intercept. Thus, the intercept point occurs at phenomenology time TREFO (see card 8 in the input deck), and all other points plotted are incremented by UNITVO (sec) multiplied by NX, where NX would indicate which time frame the RV was to be located in (see Fig, 6). This relationship cos ld be expressed as

:SFC (NT) = TREFO - NX*UNITVO, where TSEC (NT) refers to the phenomenology time at the respective RV time frame. By referring to Fig. 6 , it can be seen that for negative $\mathrm{NX}$ the pilenomenology time is increasing as the RV's get closer to the impact (relative to the burst trajectory intercept point).

The azimuth variations in this mode are controlled by the input variables NIUXO and NPXO. NMXO UNITHO deg (NMXO printout columns) are scanned to the left of the reference tra: ctory, while NPXO * UNITHO deg (NPXO printout columns) are scanned to the right of the reference trajectory. 


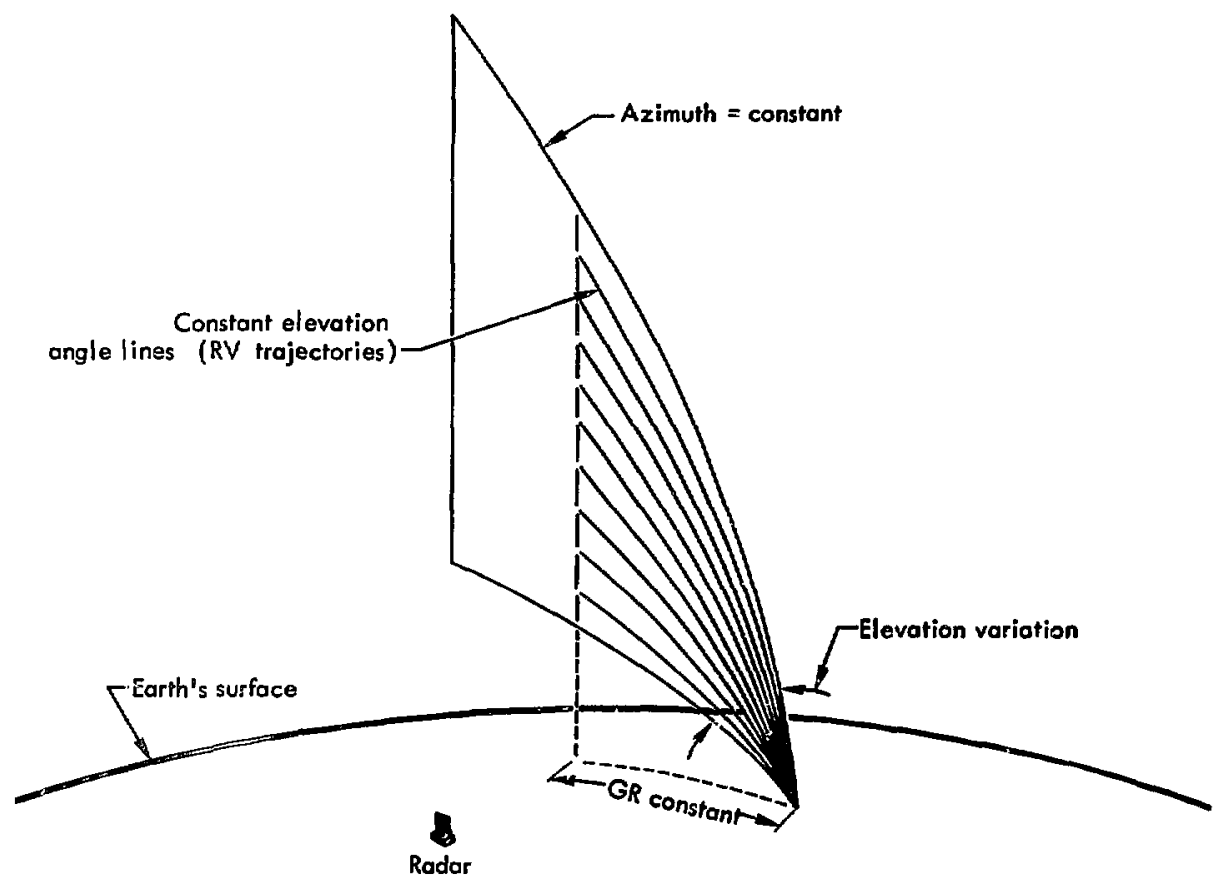

Fig. 7. PALLOT elevation mode.

\section{ELEVATION MORE}

The elevation mode keeps the trajectory ground range and azimuth angle constant while varying the elevation angle. (See Fig. 7.) The elevation mode is selected when the switch LFL is set equal to two. Like the azimuth mode, the elevation mode operates in two formats: phenomenology time constant and phenomenology time changing. The operation of these formats is analogous to the operation of the phenomenology time formats in the azimuth mode. If TMADV equals zero, the phenomenology time, TSEC (NT), is frozen for all PALIOT calculations. If, however, TMADV equals one, then phenomenology time changes from RV time frame to RV time frame (see Fig. 8). The phenomenology time is represented by the relationship

TSEC $(N T)=$ TREFO - NX UNITVO

where NX indicates the time frame in which the RV is located. It can be seen from Fig. 8 that for a negative NX, TSEC (NT) represents an increasing phenomenology time as the $R V$ approaches the impact point. Similarly, for a positive NX, phenomenology time cucreases as the RV moves away from the burst point 
reference trajtctory intercept - until, of course, TSEC (NT) becomes zero, whereupon the RV glides through a benign environment.

The elevation angle in this mode is controlled by the input variables NMXo and NPXO. Using the trajectory with launch angle equal to VFPEO deg as the reference trajectory, this mode moves NMXO o LNITHO deg (NMX? printaue columns to the left) below the reference trajectory and NPXO UNTHO deg (NPXO printout columns to the right) above the reference trajectory.
INLINE MODE

The most compliented of the threx PALLOT modes is the inline mode (Ifitil). (See Fig. 9.) This mode was wrilten to study the effects of multiple liV's lorated on the same trajectory. but spacri a certain time (UNITHO) apart. The NV's are allowed to move along the trajectory in constant time increments (UNITVO) (sce Figs. 9, 10, and 11). RANC IVA calculations are performed an the RV's along the trajectory after each incrementation of UNITVo. Further detail is needed to

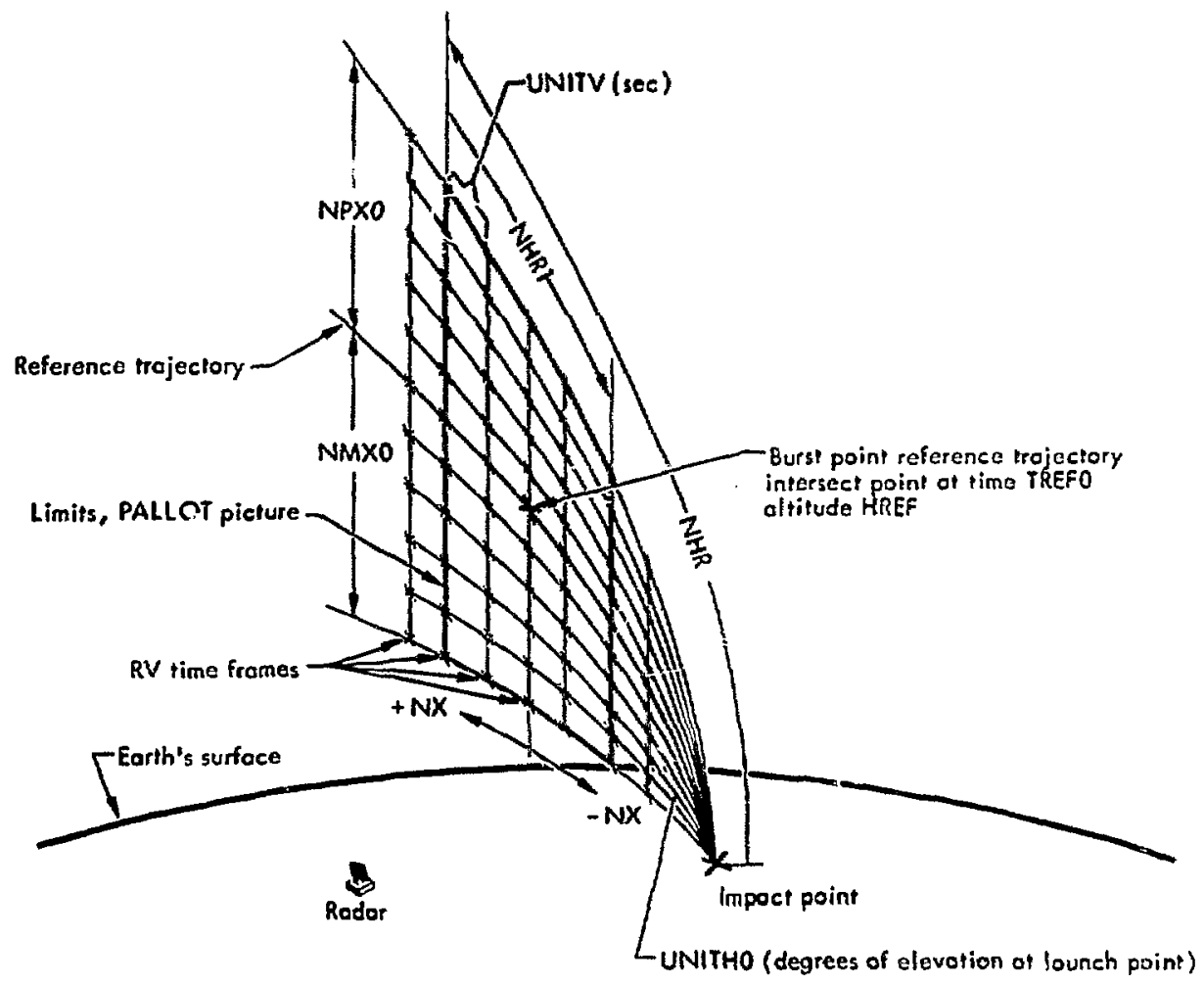

Fig. 8. Detalled view of the PALLOT elevation mode. 


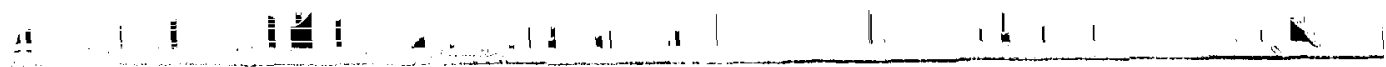

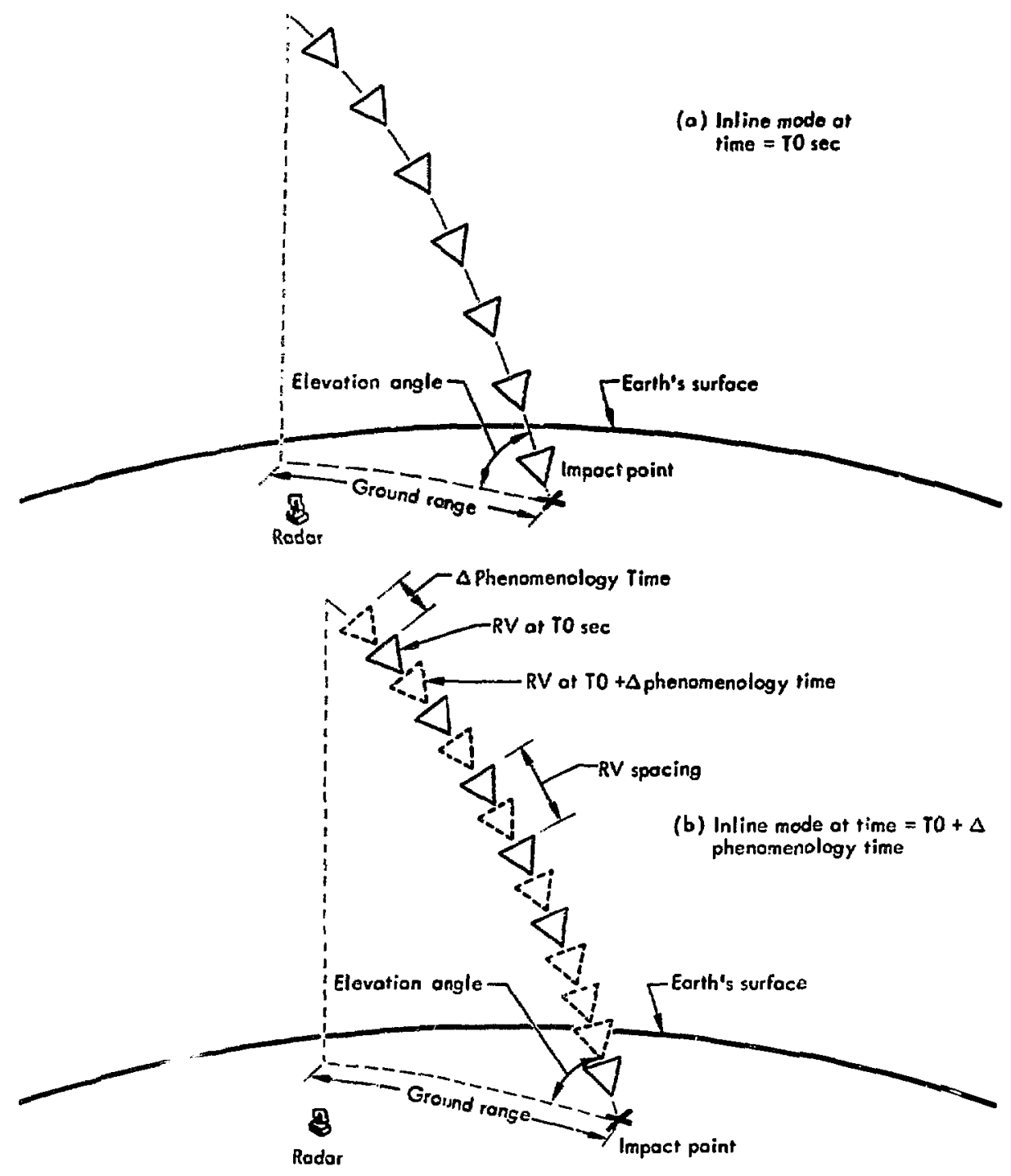

Fig. 9. PALLOT inline mode. 


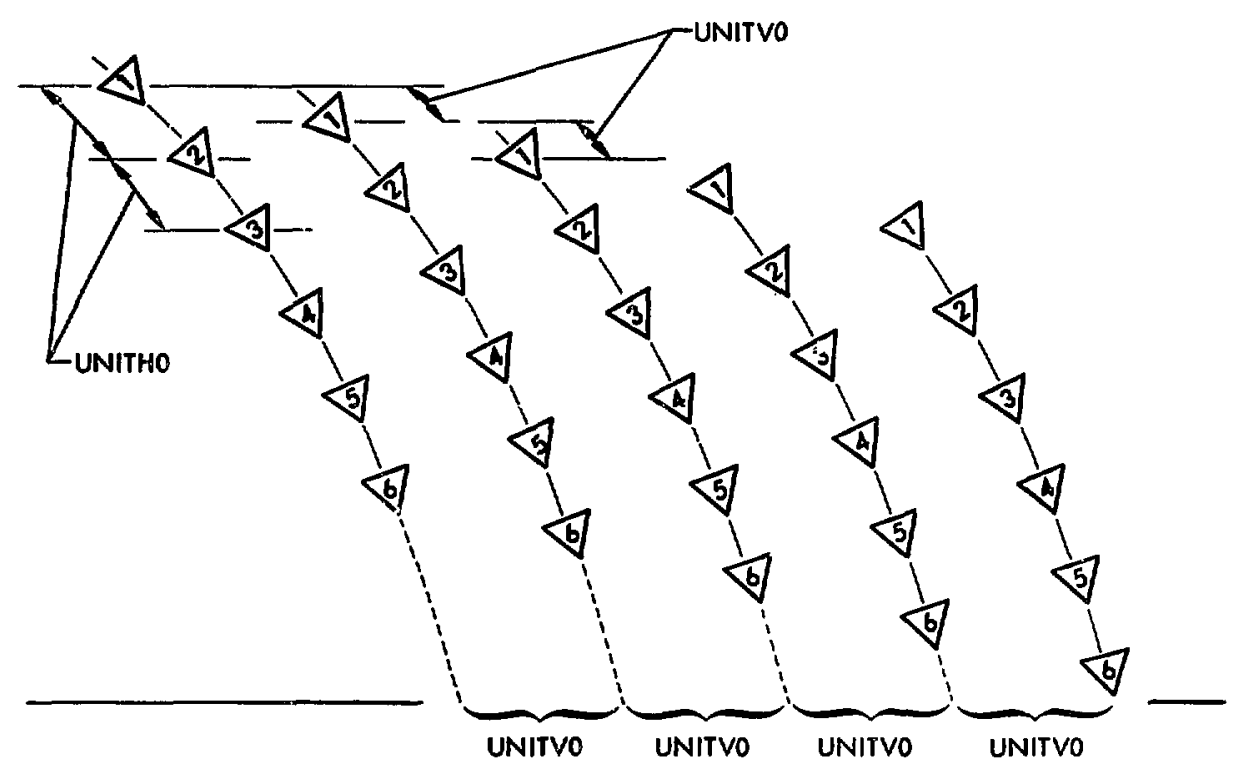

Fig. 10. PALLOT inline mode RV configuration.

explain the intricate workings of the inline mode. First, the inline mode with phenomenology time frozen will be described, then the cese with changing phenomenology time will be discussed.

\section{Constant Phenomenology Time}

PALLOT's pictures represent a set of RV's on a particular trajectory for a constant phenomenology time (TSEC = constant). An inline mode picture is formed by the following process:

(1) The RV's are taken as they are positioned along the trajectory at time TREF0, and these conditions are congidered ap defining a reference trajectory. The reference $R V$ is located at HREF at time TREFO. (Refer to Fig. 11.)

(2) PALLOT then moves up the trajectory NHR * UNITVO sec from the impact point and relocates the reference RV at this position with NMXO RV's below it and NPXO RV's above it.

(3) Next, PALLOT proceeds to move this set of RV's down the trajectory in increments of UNITVO sec until it has completed NHR 1 increments. Thus, PALLOT produces an $n * m$ matrix of RANC IVA calculations where

$$
\mathrm{n}=\mathrm{NHR} 1 \text { and } \mathrm{m}=\mathrm{NPXO}+\mathrm{NMXO} \text {. }
$$

\section{Incremen ing Phenomenology Time}

This mode, as would be expected, is similar to the preceding mode. The phenom enology time TSEC (NT) is set equal to TREFO - DVT, where DVT is the number of rows above the reference tra ectory row. (See Fig. 11b.) Then, concurrent with the movement of the RV's 
down the trajectory in increments of UNITVO sec, the phenomenology time is increased in increments of UNITVO sec.

\section{COMPLETE TIME HISTORY MODE}

When it is desirable to observe the effects of a burst along a specific trajectory

(o) Inline mode reference trojectory

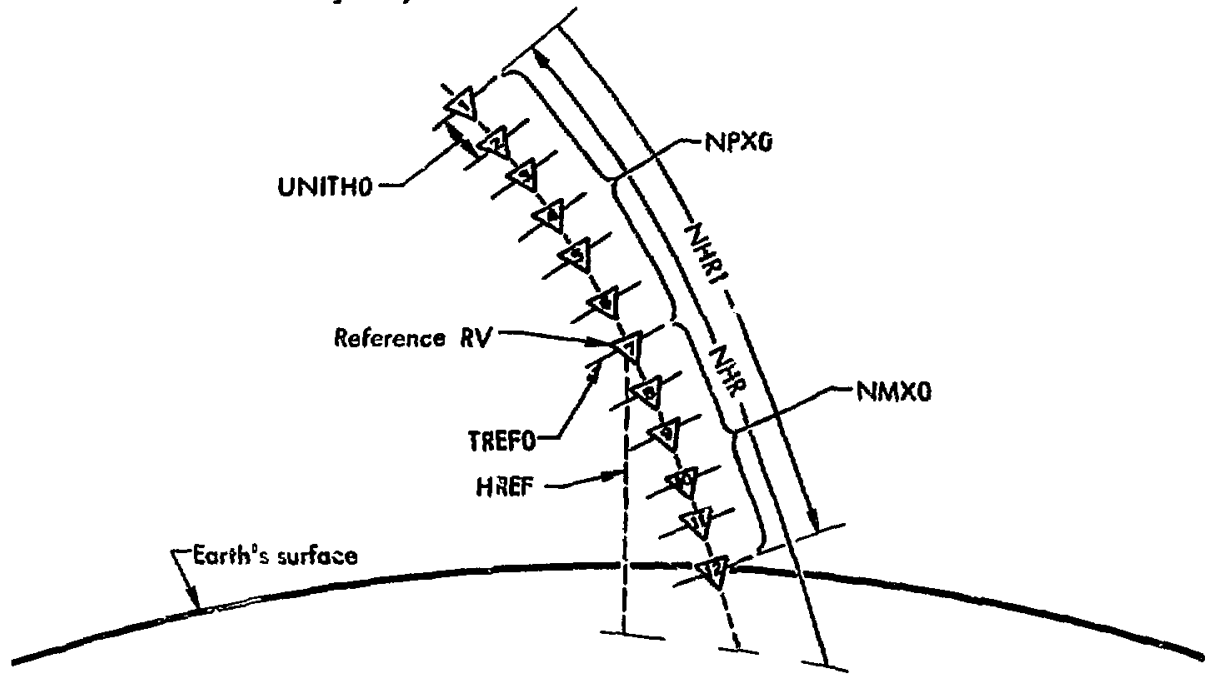

(b) PALLOT picture output matrix

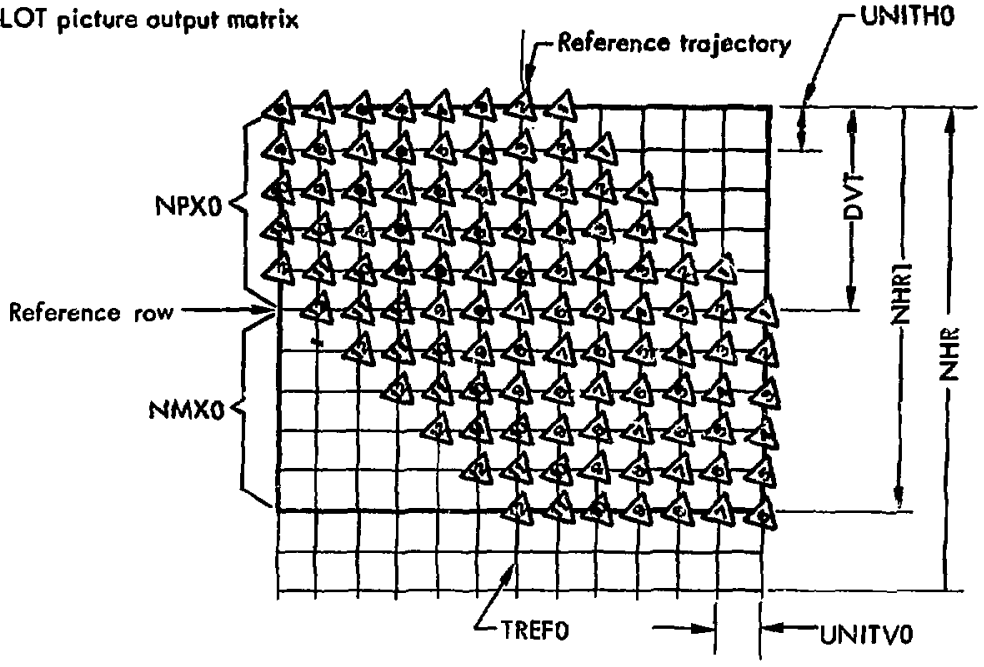

Fig. 11. Detailed view of the PALLOT inline mode. 


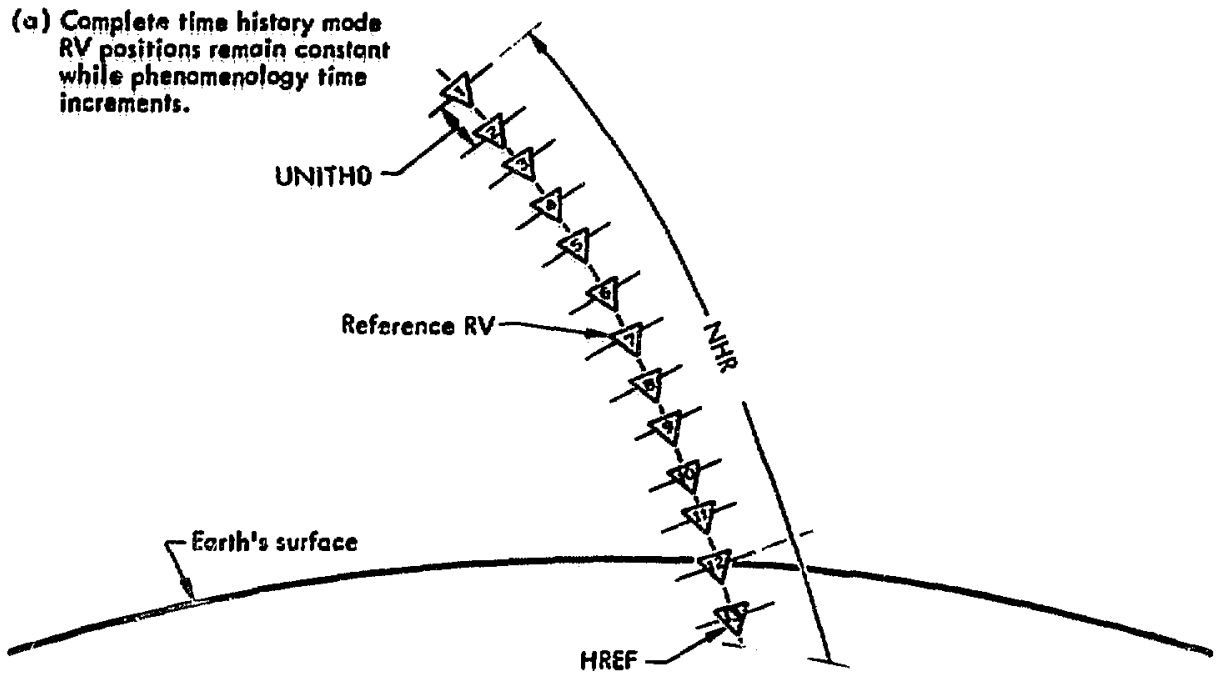

(b) PALLOT piçure output motrix

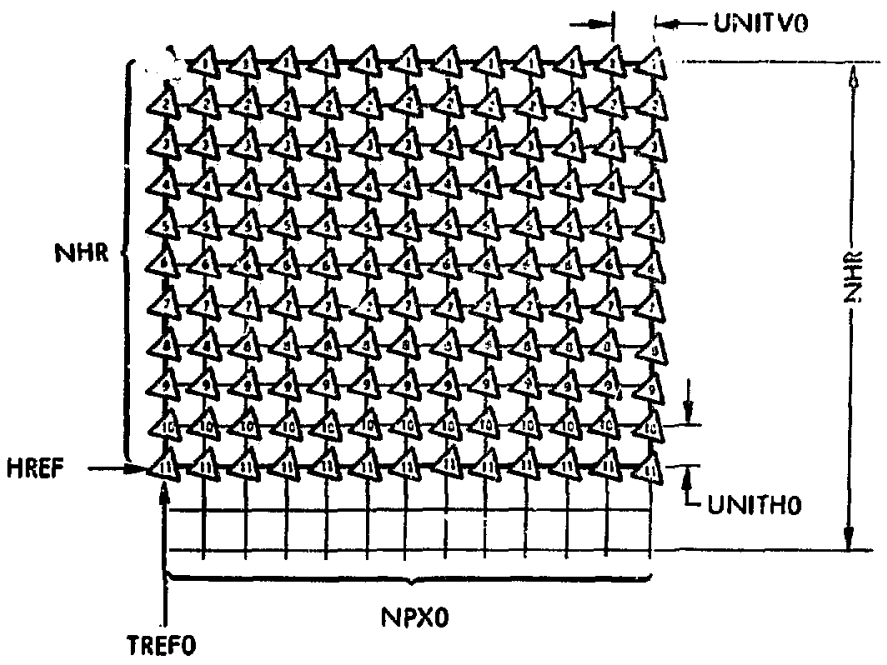

Fig. 12. PALLOT complete time history mode RV breakdown. 

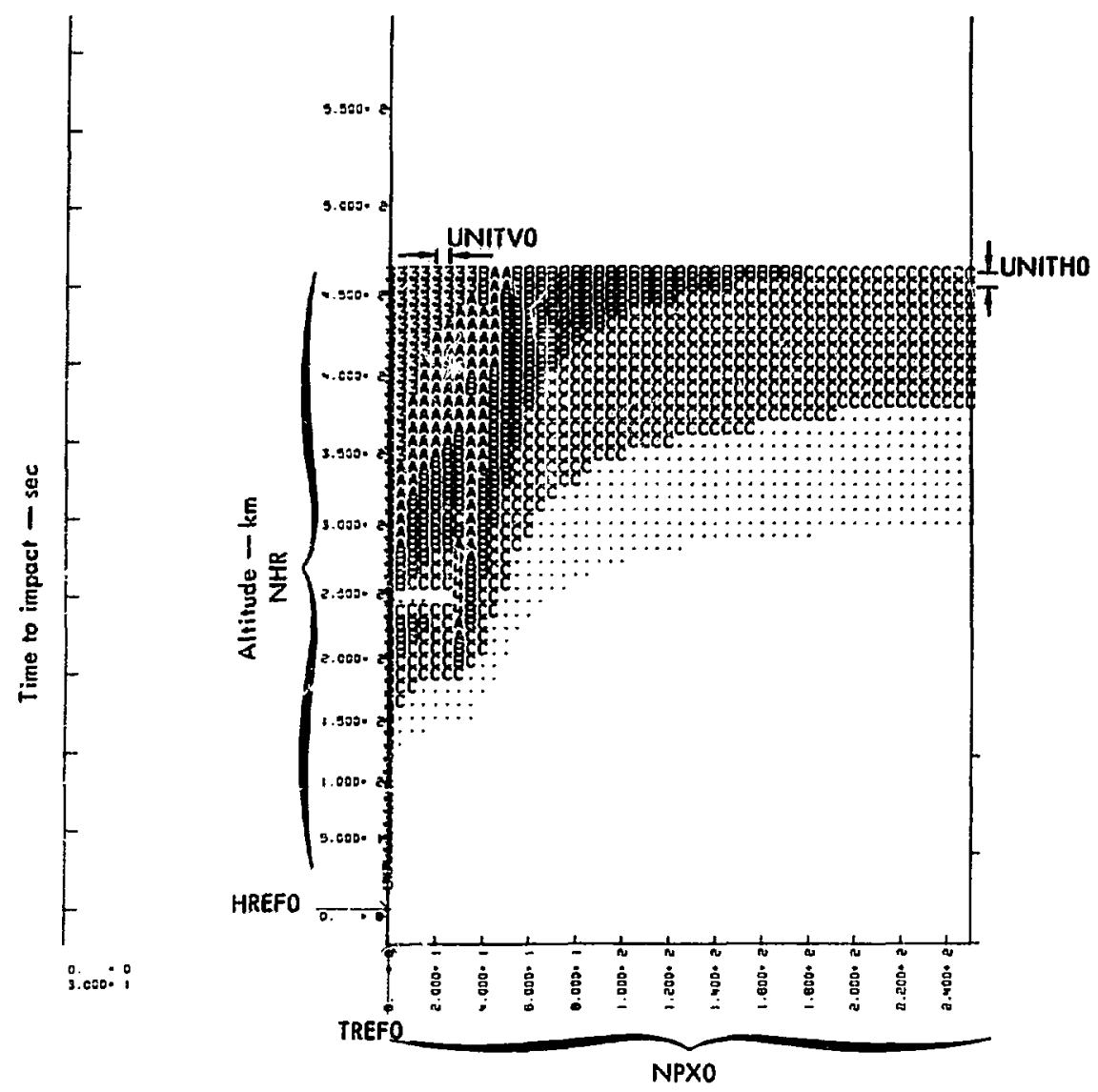

Phenomenology time - sec

Fig. 13. PALLOT complete time history mode.

for a particular set of phenomenology times, the complete time history mode is used (setting IFL $=3$ ). This mode allows the user to select portions of the RV's trajectory and freeze that trajectory for any set of phenomenology times. The controlling variables of this mode are shown in Fig. 12. Figure 13 shows the $\mathrm{OZ}$ overlay of a complete time history run. NHR is the number of RV's above the reference altitude HREFO $(\mathrm{km})$. UNITHO is the RV separation in seconds. NPXO is the number of phenomenology calculations to be made after TREFO sec; NMXO is set equal to zero in this mode. Finally, UNITVO is the increment of time between phenomenology calculations, and it must equal UNITHO in this mode. The complete time history mode sweeps time from TREFO sec to TREFO + NHR 
* UNITVO sec, and the altitude from HREFO km to HREFO + NPXO * UNITHO * $(x \mathrm{~km} / \mathrm{sec}) \mathrm{km}$. ( $X$ is a factor dependent on the selected trajectory and is internally calculated in the code.) This mode has been found extremely useful in analyzing the blackout effects of a burst on reentry trajectories. A sample of PALL'T's complete time history mode is shown in Appendix 3.

\section{Overlays}

PALLOT has two overlays $1 \mathrm{CZ}$ and TY) that operate on its various outputs; overlay $O Z$ can be used with all four modes. The only rule that need be remembered in using these overlays is that the rows to be printed (using the dd80, a dieplay device at the Lawrence Livermore Lahoratory) do not exceed 80 in number and 60 columns in length.

Samples of OZ working on the various modes are shown in the appendices. However, a second overlay was written for the inline mode because the $\mathrm{OZ}$ overlay for this mode is confusing. The TY overlay performs a matrix manipulation of the final PALLOT pictures to present constant altitude lines horizontally on the do80 plots. A comparison of the two overlays for this mode will give the user a quick appreciation of this second overlay.

\section{Acknowledgments}

The author wishes to acknowledge the invaluable assistance of Ross Castleton, who programmed the PALLOT code, in the collection and composition of the in- formation contained in this document. The author also greatly appreciated the constant reviews, critiques, and suggestions of D. R. Dunn and W. A. Turin. 


\section{References}

1. D. R. Dunn and R. N. Castleton, PALLOT: Ponder ALong Locus Of Trajectories, Law rence Livermore Laboratory Rept. CX-72-73 (1972) (title U, report SRD).

2. D. R. Dunn and R. N. Castleton, PALLOT, A Code for Displaying Nuclear Device Effects Along Reentry Vehicle Trajectories From RANC, Lawrence Livermore Laboratory, to be published.

3. L. L. Cleland and R. N. Castleton, Spatial Pictures of Radar and Nuclear Weapon Interactions, Law rence Livermore Laboratory Rept. UCR:-50813 (1970) (iitle U. report SRD).

4. L. L. Cleland, R. N. Castleton, PONDER: Pictures Of Nuclear Device Effects from Ranc, Law rence Livermore Laboratory Rept. UCRL-73043!1970) (title U, report SRD).

5. L. L. Cleland, R. N. Castleton, and D. R. Dunn, PONDER Data: Fir zion Fraction Effects and RANC III 1/2-IV Comparisons, Lawrence Livermore Laboratory Rept. UCRL-51384 (1973) (title U, report SRD).

6. M. D. Maples, PONDER: A Fortran Code Which Graphically Represents Nuclear Blackout Effects, Lawrence Livermore Laboratory Rept. UCRL-51391 (1973).

7. R. I. Bogusch et al., RANC IV: Computer Simulation of Radar Propagation in a Nuclear Environment, General Electric-TEMPO Rept. 70 TMP-37 (DASA 2497) (1070) (title U, Vol. I, CFRD; Vol. II, U; Vol. II, SRD). 


\title{
Appendix 1
}

\section{PALLOT Listing}

\author{
PALLOT
}

This program uses RANC IVA as a subroutine to calculate nuclear blackout effects along a family of reentry trajectories. Subroutines SPRAY and TRAJECTORY are used to produce this family of trajectories.

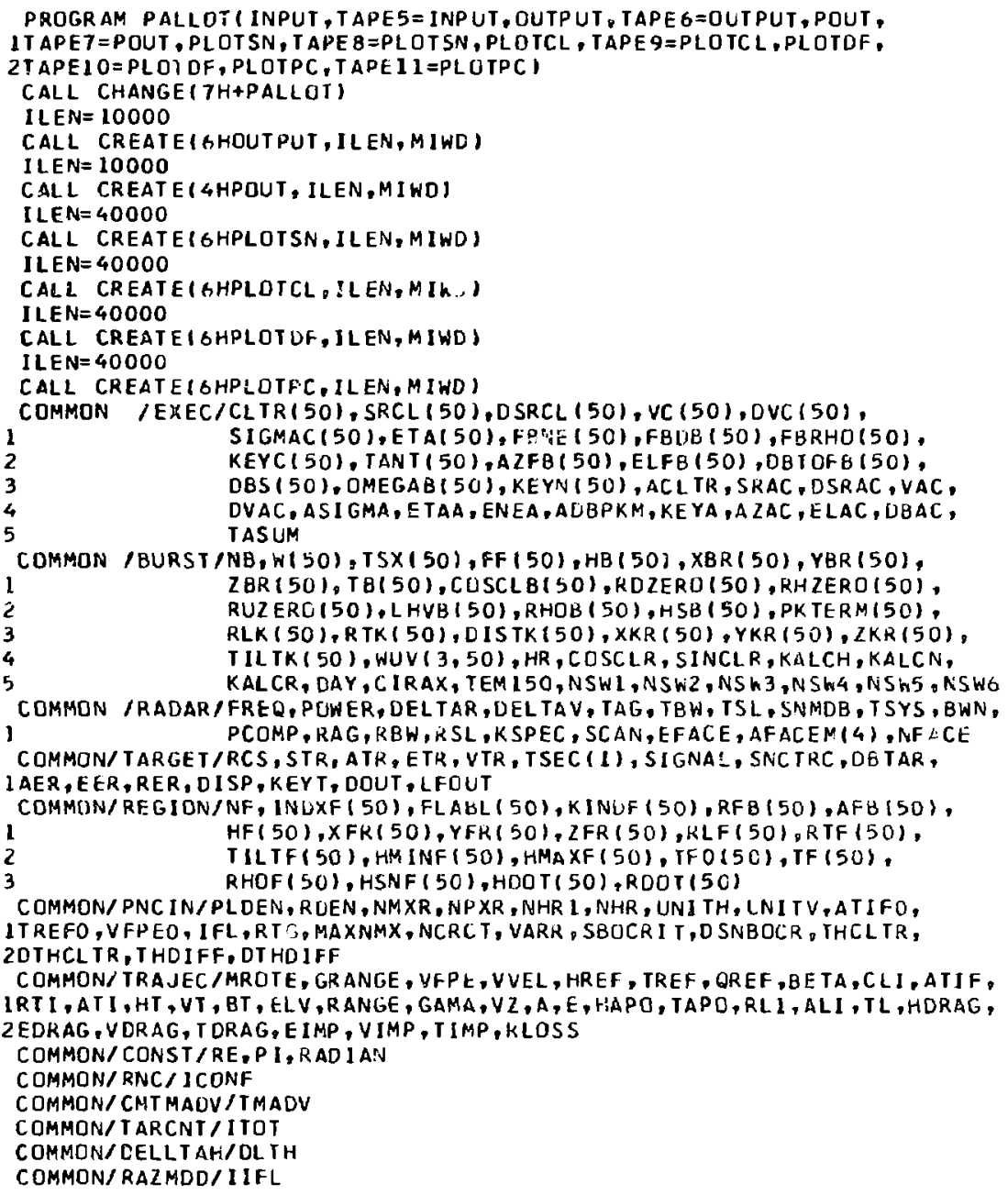


OINENSIOA, SAPRES( SUUO), SNPREV (SUU), CLPRES $(500)$,DFPRES(500), IPCPRES(500), KYT(

NPGIUT $=7$

NPI. TSN=8

NPLTLL $=9$

NPLTDF $=10$

NPLTPL=11

I C C.NF $=1$

GO TU 3

I $I C L V F=2$

3 CALL KANC

KTG $=100000$.

I AREA $=0$

AREA $\mathbf{I}=0$.

$\triangle R E A O=0$.

$M \times T$ ANT $=0$.

$M \times O B T F=0$.

$M X \subset B S=0$ 。

$M X T F=0$.

$M X F B R=0$ 。

$M \times F B E=0$.

$M X F B D t=0$.

$1 \mathrm{TOT}=0$

SNBOCR = SBOCR IT + CSIBBUCR

CLBOC $=$ THCLTR-DTHCL IR

DFBOC $K=$ I HOIFF+DTHCIFF

UNITYO=PLDENHUNITV

UNI THO $=$ PLDEN*UNITH

UNITVR= RDEN*UN ITVO

UNI THR = ROEN WUN IT HO

WR I TE (NPOUT, 3003 ) UN I THR, UN I TVR, UN I THO, UNI TVO, UNI TH, LNI TV, NNXR,

INPXR, NHRI , NHR, MAXNMX, AT IFO, RDEN, DL TH, TREFO, HREF, QKEF, GRANGE,

2VFPEO, VVEL, BETA, CLI, MROTE, SBUCR IT, OSNBOCR, THCLTR, OTHCLTR,

3THOIFF, CTHOIFF, IFE

I PLDEN=PLLEN

I RUEN $=$ RCEN

I RP = IRLEN I PL CEN

NMXIRC $=N M X R * I R D E N$

$N P X I R L=N P X R *] R[E N$

MAXNRV $=\left(N M X_{R}+N P X_{K}\right) \neq I K P+1$

OU $81001=1$, MAXNKV

PCPRES $(I)=0$.

CLPRE $S(I)=0$.

DFPRES $(I)=0$.

SNPRE $\triangle(I)=0$.

6100 SNPREV(I) $=0$.

I PLOT $=2$

I RUN $=2$

IJPRES $=0$

$N L P R E S=0$

NRPRE $S=0$

ELREF $=N H R I$ HUNITVR

DO $6000 \quad i 1=1$, NHR

GO TC $(7001,7002)$, LRUN

7002 GO TO(7701,7702), IPLUT

7702 CONTINUE

I PLOT = I

I RUN $=1$

$N M X=N M X R$

$N P X=N P X R$

UNH $=$ UNIIHK

TOUI CONTINUE

ELREF $=E L R E F-U N I T V K$ 
$10=1$

GO TO 7003

770

CONT I NUE

ELR $=E$ L REF

UNH=UNITH

$I 0=I R P$

7003 DO $600312=1,10$

GO TO(7006,7005), IRUN

1005 ELREF $=E L R+(1 R P-12) * U N I T V$

ILNCT $=0$

I RNCT $=0$

7006

CONT INUE

$I L=0$

I $R=0$

I LEFT $=1$

$N R=N M X+1$

$N R Y=N M X+N P X+1$

NRV PR EV $=0$

IF(IIFL.EO.1) GO TO 9999

IF(TAADV.NE.0.) TSECII)=TREFO-ELREF+DLTH

3999 CONTINUE

770? CONTINUE

DO 309 ITRGT $=1$, NRV

ICGNF $=3$

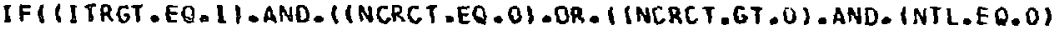

1. AND。(NTR。EQ。0)) - AND. (TMAOV.NE.0. ) ICONF $=4$

GD $1015001,5002,50031$, ILEFT

.001 CONTINUE

$N R=N R-1$

GO TO 5004

$\because 06 \cdot$ CONT I NUE

$N R=-N P X$

I LEFT $=3$

GO TO 5004

, 003 CUNT INUE

$N R=N R+1$

DOQG CONTINUE

CALL SPRAY (RTG, UNH, NR, ELREF, AT 1FO, TREFO, VFPEO, IFL, VARR, RCS)

IF (RTG.GT .0.) GO TO 1901

$S I G N A L=S N B O C R+100 C$.

SNCTRC $=$ CLBOCR -1000 .

GO TO 1902

1901 CONTINUE

IF(IIFL.EQ.1) TSEC(I) = TREFO+(ITRGT-I) *UNITVO

CALL RANC

DO 8800 INB $=1$, NF

MXT ANT $=$ MAX LF(MXYANT. TANT(INB)

MXDBT $F=M A X 1 F(M X D B T F$, DB TOFB (INB) )

$M \times D B S=M A X I F(M \times D B S, D R S($ INB $)$

$M X: F=M A X I F(M X T F, T F(I N B))$

$M X F B R=M A X$ IF (MXFBR, FBRHOIINB )

MXF BE $=$ MAX IF(MXFBE, FBNE(INB))

$M X F B D B=M A X I F(M X F B D B$ * FBDB(INB)

8800 CONTINUE

1902 CONT INUE

DIFF = SI GNAL-SNCTRC

SNPC $=10 . * *(-01 F F / 10)+.10 . * 1-51 \mathrm{GNAL} / 10$.

SNPC $=-10 . *$ LUG 10F (SNPC)

מOO5 CONTI UE

GD TC!5005, 308), IRUN

IF( ( YPC-SNBOCR) .GE.0.1.ANO.( (SIGNAL-SNBOCR).GE.0.)) GD T0 309

5006 GU TI 2007,5008,5008), ILEFT

9007 ILEFI $=$ ? 


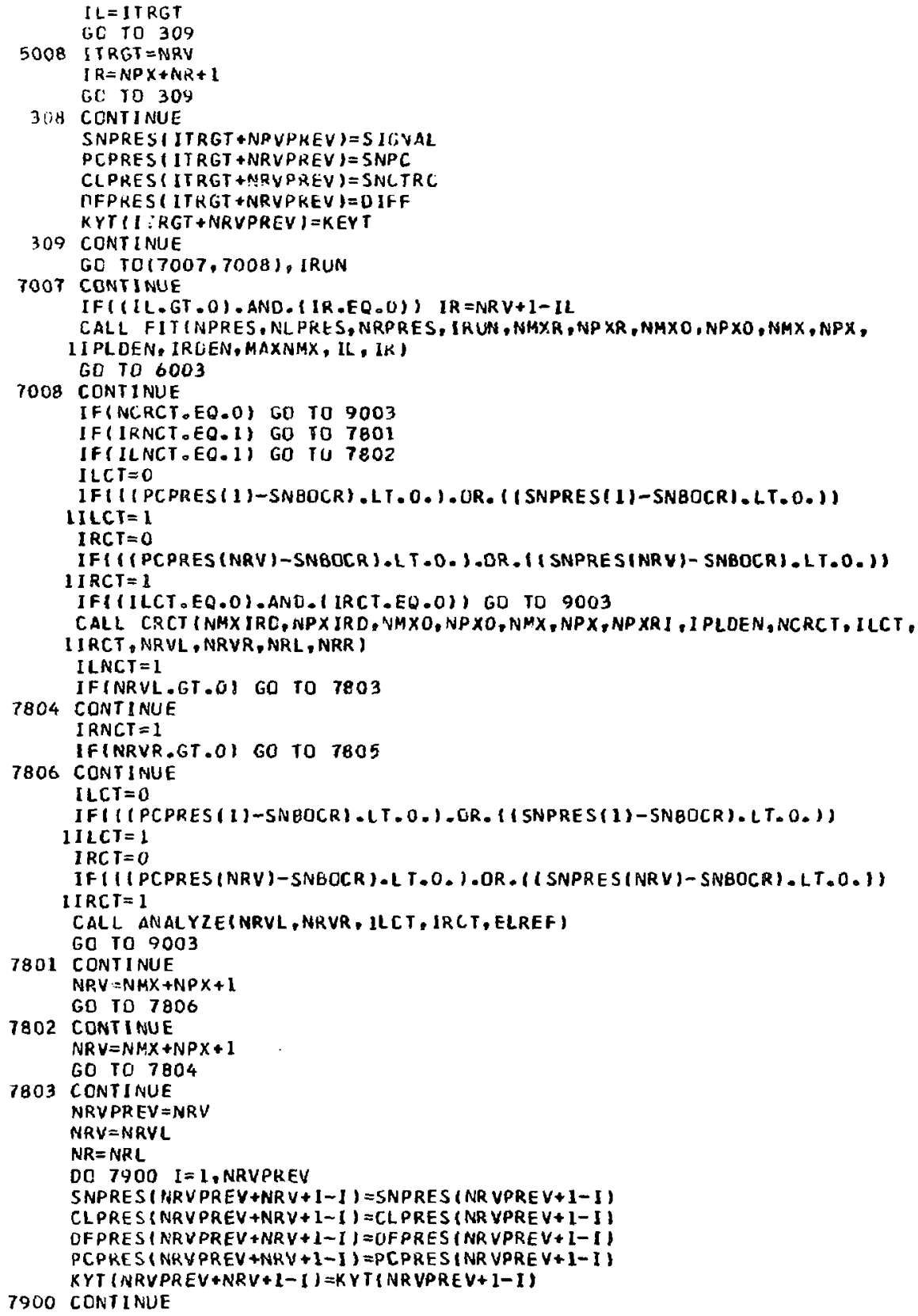


NRV PREV $=0$

GO TO 7703

7805 CONTIIUE

NRVPREV $=$ NRV

NRV $=$ NRVR

$N R=N R H$

$M P X=N P X R I$

GO TO 7703

9003 I $3=\operatorname{MOL}(12$, (PLDEN)

IFI13) $18001,8000,8001$

8000 CALL PLCTSNISNPRES, KYT, SNBDCK, LSNEOCK, NPXO,NMXO,U, O,MAXNMX, IPLEE (NPLTSN)

CALL PLCTSNIPCPRES, KYT, SNBOCR, USNBOCK, NPXO,NMXO , O , O, MAXNMX, I PLOEN, INPLYPC)

CALL PLOTSNIDFPRES,KYT, OFBOCR, DTHOIFF, NPXO,NMXO,0,0,MAXNMX, IPLCEN, I NPLTUF)

CALL PLCTCICLPRES, KYI, CLHOCR, UIHCL TR, NPXO, NMXO, 0, O, MAXNMX, I PLDEN, INPLTCLI

I PLOT $=2$

80U1 CALL AREALPCPRES, SNPREV, SBOCRIT, UNITH, UNI TV,NMX, NPX, NRV, MAXARV, II LKEA, AREA I, AREA(S)

6003 CCATINUE

IF(IIRUN.EQ.2).ANE.(IPLDT.EQ.2)\} GO TU 7701

6000 CONTINUE

AREAAV = $\mid$ AREAI $\therefore+A-A O) / 2$.

$A A=A R E A A Y \div(S T M+2)$

IF (AREAI) $3501,3502,3501$

3502 APPERR $=100$.

$60 Y 0 \quad 3507$

3501 APPERK $=($ AREAO/ AREAAV-1. $1 * 100$.

3507 AI=AREAI* $(S T R * 2)$

$A C=A R E A C \div(S T R * \# 2)$

IF(TMADV.EQ.0.) WRITE(NPOUT, 3005) TSECII)

DO $3503 \quad I=1, N B$

3504 CONTINUE

IFITB(I)-TSEC (1) $13504,3504,3503$

CALL COCROX(0, 2, XBR(I),YBR(I), ZBR( 1$), \times 1, A B R, \times 2, \times 3, \times 4, \times 5$,

ISBR, EGRI

WRI TE (NPOUT, 3006) TB(I),W(I),FF(I),TSXII),HB(I), SBR,EBR, ABR

3503 CONTINUE

WR ITE INPOUT, 3007) SBOCR I T, RC.S, FREO

WRITE (NPOUT, 3008 ) AREAAY, APPERR

MRT E $=A B S F(M R O T E-1)$

$M A D V=T M A O V$

WR I TE INPOUT, 3030 ) MRTE, GRANGE, VFPEC, VVEL, HREF, TREF C , BE TA

WR I TE INPQUT, 3031 ) HDRAG, TDRAG, VDRAG, EDRAG

WRITE (NPOUT, 3040) ITOT

TANT $(1)=$ MXT ANT

OBT OF $B(1)=M \times O B T F$

DBS $(1)=\operatorname{MXDBS}$

$\operatorname{TF}(\mathfrak{l})=\operatorname{MXTF}$

FBR $H O(1)=M \times F B R$

FBNE(1) = MXFBE

$F B D B(1)=M X F B C B$

CALL RNCGT

WRITE (NPLT SN, 3009 )

WR I TE (NPL TCL, 3009)

WRITE (NPL TDF, 3009)

WRITE (NPLTPC, 3009 )

WRI TE (NPOUT, 3009 )

GO TO 1

3001 FORMAT(120A1)

3003 FORMAT(31/2r 20.10$), / 5120,31 / 3 F 20.10), / 2 F 20.10,110,3(/ 2 F 20.10), / 110$ 1) 
SCOS, FCRMAT (F5) - IO)

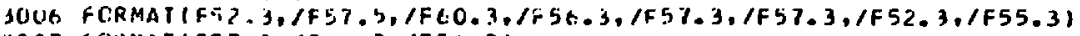

3007 FCKMAT (F57.3./F40.3,/F50.3)

3006 FCAMAT(F54.6,/F41.

SCLO FCAMAT (3HENC)

3030 FCAMAT $1 / 1 / 1 / 2,2 x$, ot 12.4$)$

3031 FCKMAT $(14 \times, 4 F(2.4)$

3040 FCRMAT (11U)

inO 
ISYS $=\operatorname{AMAX} 1(\times(2), 1$.

$B W N=A M A X 1(X(3), 1, E-3)$

$P C O M P=X(4)$

$R A G=x(s)$

$R B W=X(6)$

$\mathrm{RSL}=X(7)$

$\triangle L P H A Z=A L P H A$

GO TU 1

$31 K S P E C=x(1)$

$S C A N=X(2)$

$E F A C E=X(3)$

AFACE $(1)=X\{4\}$

NF ACE $=1$

DD $33 I=5,7$

IF $(X(1) 132,33,32$

32 NFACE $=$ NFACE +1

AFACE (NFACE) $=X(1)$

33 CONTINUE

IF ( SC AN ) 34, 34, I

14 NF $A C E=0$

GO TO 1

$\therefore 1$ GLATO $=x(1)$

GLONO $=X(2)$

DAY $=X(3)$

KALC $=X(4)$

$K A L C H=M A X O(1-H O D(K A L C, 1000) / 100,0)$

$K A L C N=M A X O(1-M O D(K A L C, 100) / 10,0)$

$K A L C R=M A X O(1-M O D(K A L C, 10), 0)$

$K O U T=X(5)$

KTRACK=MINI $(\operatorname{ABS}(x(6)), 10$.

$K O D E=K(7)$

NSW $1=$ MOD $(K O D E, 1000000) / 100000$

NSW2 $=$ MOC ( KODE, $100000 \% 10000$

NSW $3=\operatorname{MOC}(K O D E, 10000) / 1000$

NSW4=MOD( KODE, 1000$) / 100$

NSW5 $=$ MOC $(K: U E, 100) / 10$

NSWG $=$ MOC $(K U D E, 10)$

IF (NSW6.EQ.1) NSW $5=1$

I F (NSW5.FQ.1) NSW4=1

IF (NSW/4.EQ.1) NSW 3=1

GO TO 1

51 I F (KODE) $52,53,52$

52. CALL COORDX(0, KOCE, X(1),X(2), X(3),RRO,ARO, HR, XRO, YRO, 2RO,SRO,ERO) GO TO 54

$53 \mathrm{GLATR}=X(1)$

GLONR $=X(2)$

$H R=X(3)$

54 KLR $=K O D E$

$D I P R=X(4)$

$V A R R=X(5)$

CALL COOROX(0,., $, X(6), X(7), 0,0, R I 0, A I 0, D U M I, X 10, Y I 0,210,5 I 0, E I C)$

ALPHA $5=$ ALPHA

$H H R=H R$

GO TO 1

61 IFIIN6) $62,65,62$

62 IFI ING-50)63,69,63

63 IF $X(4)-T B(N B)) 64,65,65$

64 NHELP $=63$

65 IN $6=$ IN $6+1$

$N B=I N 6$

IF ( KODE )66,67,66

66 CALL COCRDX(0..KODE,X(1),X(2),X(3),RBO(NB),ABO(NB),HB(NB),XBO(NB). (YBO(NB), $Z B D(N B), S B O(N B), E B O(N B)$ ) 
60 To 68

67 GLATB(NE) $=K(1)$

GLONB $(N B)=x(2)$

$H B(N E)=X(3)$

$68 K L 8(N A)=K O D E$

$T B(N B)=X(4)$

$W(N B)=X(5)$

$F F(A B)=X(B)$

$\operatorname{TSX}(N B)=X(7)$

ALPHAG $(N H)=A L P H A$

GO TO 1

69 NHELP $=62$

GO 701

71 TSEC (NT $)=X, 1)$

MAXNMX $=X(2)$

NCRCT $=X(3)$

SIGMA $=X(4)$

MROTE $=X(5)$

WREF $=x(6)$

DLTH $=X(7)$

IFL $=K G C E$

I I FL $=0$

IF(IFL。EG.3) I I FL = I

I $F($ I I FL。EQ. I) IFL = I

GO TO 1

81 ATIFO $=\{(1)$

GRANGE $=I(2)$

VFPEO $=x(3)$

VVEL $=X(4)$

$H R E F=X(5)$

TREFO $=X(b)$

BET $A=X(7)$

THADV $=$ KCDE

GC TO 1

$\because 1$ CONT INUE

IF $(K O D E$. NE.O $)$ GU TO 92

$P L D E N=X(1)$

RDEN $=X(2)$

UNI $T H=X(3)$

UNITV $=X(4)$

NMXR $=X(5)$

$N P X R=X(6)$

$N H R=X(7)$

UNI TH=UNITH\#PLCEN

UNI T $V=U N I T V \neq P L D E N$

$N M X R=N M X R / R D E N$

$N P X R=N P X R / R D E N$

NHR $=$ NHR /ROFN

PLDEN=1./PLDEN

NHR $1=N H R$

IF(NHR,LT.100000) GD TO 1

NHR $1=$ NHR $/ 100000$

$N H R=N H R-N H R I * 100000$

GO TO 1

92 CONTINUE

SBOCR IT $=X(1)$

$D S N B O C R=X(2)$

THCL T $R=X(3)$

DTHCL TR $=X(4)$

THOIFF $=X(5)$

DTHOI $F F=X(6)$

GC TO 1

101 CONT INUE 
HRITE CUTPUT TAPE $6,40 C 9$

IFI"JCPCT.EQOC) WRITE OUTHUT TAPE G.4U15

[F(KLK) IUL, 103, 10L

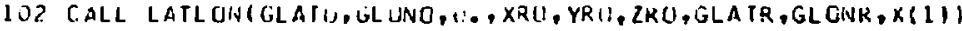

GD TU $1 \mathrm{C} 4$

103 CALL XYLIGLATO,GLCNU,0, GLATR, GLOLR,HR, XRR, YRO, ZRC)

CALL CGCKOX (0, 2, XKU,YRL,ZRO,KKU, ARO, XI1),XRU,YRO,ZRU,SRC,ERUI

114 IFINGIIOS, 110,105

1 is DC $10 \theta 1=1$, NB

IF(KLHII) IOG, 10/,1UK

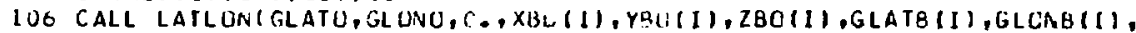
$(x(1))$

GC TE $10 \mathrm{H}$

$10 ?$ CILL XYZ(GLATG, GLCNU, O.,GLATB(1), GLOVB(1), HBII),XBO(1), YBDII), $1 \ell \mathrm{BC}([))$

CALL CCCKOX(0.,2, XUU(I), YBO(I), 2BO(I), RBCII),ABCII),X(1),XBC(I),

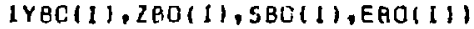

108 CGNTINUE

110 IFINRVIIII, 115, 111

$111001141=1$, NRV IF(KLT(1) I112,113,112

112 CALL LATLONIGLATU,GLOND,O, XTOII), YTO(1),ZTOII),GLATI(I),GLCAT(I). (X) (1)

GC TO 114

113 CALL XYZIGLATO,GLGNO,O, , GLATT(I), GLDNTII),HTII), XIOII), YTO(I), $12 \mathrm{JO}(\mathrm{I})$

CALL COCRDX(O.,2,XTU(I), YTOII),2TU(I),RTO(I),ATOII),X(1),XTC(I), IYTO(I), 2TOIII,STU(I),ETU(I)

114 CONTINUE

115 CONTINUE

CALL LATLON(GLATU,GLONO,O, XIO, YIO, ZIO,GLATI,GLCNI, X(1) I

$C L 1=(90 .-G L A T$ I $) \supsetneqq R A O I A N$

COSCLI $I=\operatorname{COS}(\mathrm{CLI})$

S JNCL I $=$ SIN(CLI)

$C L R=(90 .-G L A T R) \div R A D I A N$

$C O S C L R=\operatorname{COS}(C L R)$

$S$ INCL R=SIN (CLR)

CSCLK=CCSCLR

SNCLR = S INCLR

CLO $=(90 .-G L A T D)$ * RADI AN

$\operatorname{CCSCLO}=\cos (\mathrm{CLC})$

SINCL U =SIN ( CLO)

CALL ASPECTICOSCLD,SINCLU, 0, XKU, YRU,ZRU,I, XIO, YIC, ZIO,XIR, YIR, IIIRI

CALL ASPLCT ICGSCLR,SINCLR,HR,XIR,YIR,ZIR,I, $0,0,, 0 ., X R I, Y K I, Z R I)$ IFINB) $303,311,3 C 3$

303 CALL ASPECTICOSCLC, S INCLU, O . XKU, YRO, ZRO, NB, XBD,YBC,ZUO,XBR, YBR, [ZBR]

DO $30 \mathrm{~d}:=1, \mathrm{NB}$

CALL CCORUX(HR, 2, XBR (I),YBR(I), ZBRII),RBR, ABR,X(1),X(2),X(3),X(4), $15 B R, E B R$ )

308 CDNT LIVUE

311 IF(NRV) $312,400,312$

312 DO $317 \quad 1=1$, NRV

CALL ASPECTICOSCLU, SINCLO, 0, XRO, YRO, ZRU, 1, XIOII , YTO(I),ZTCIII. IXTR,YTR, ?TR ]

CALL COOROX(HR, 2, XTR,YTR, ZTR,RTR, ATR $(I), x(1), X(2), X\{3\}, X(4), S T R(I)$

1. FTRLI)

317 CCNTINUE

400 CCNTINUE

402 CALL ATNLSC(-LFOUT, $(,, x(1), x(2), X(3), x(4), X(5), x(6), X(7))$

CALL C(RA65I-LFUUT, $150, \ldots X(1), X(2), T E M 150, X(3), X(4), X(5), E M 150)$

$G 150=G O *(R E /(R E+150.1) * \%)$ 
CIRAX $=1 .+150 . E+5 *$ G1SO*EM150/RGAS/ TEM150

$X(1)=S I N(D I P R * R A O I A N)$

$\operatorname{COSCLR}=x(1) / \operatorname{SORT}(4,-3 . * x 11 ; * 2)$

SINCL R=SQRT $(1,-\operatorname{COSCLR} * 2)$

$\checkmark M R=V A R R * R A D I A N$

COSVMR $=$ COS (VMR)

SINVMR $=$ SIN $($ VMR $)$

IF (NB ) 702,711,702

702

D0 $703 \quad 1=1$, NB

$X(1)=X Q R(1) * C O S V M R-Y B R(1) * S I N Y M R$

$Y B R(I)=X B R(I) * S I N Y M R+Y B R(I) \neq C O S V M R$

$703 \times B R(I)=X(1)$

711 IF INRVI $712,721,712$

712 OO 713 I=1, NRV

713 ATRII $)=A T R(I)-V A R R$

721 IF (NFACE) 722,72:,722

722 DO 723 I=1, NFACE

723 AFACEA (I) =AFACE (I)-VARR

724 CONTINUE

IF (NHELP. INE.O) CALL HELP(GHRANCIN,NHELP, I)

RETURN

1001 FORAAT(I1,E9.0,6E10.0,1XI1,2XA6)

3009 FORMAT ( 3 HEND)

4009 FORHAT I 3 HIRUN COMMENTSI

4015 FORMAT 141 LNO FINE CORRECTIDNS MADE TO TAILORED GRIDI END 
This subroutine produces the target matrix point by point, from left to right, and row by row. Each target point produced by SPRAY is then checked by RANC to find its signal/noise ratio.

RTG = target shell radius.

UNH = grid horizontal increment.

NR = position along horizontal line at which target is now located.

ELREF = elevation angle (degrees cona $=0, \mathrm{~km}$ cona $=2$ ) set to topmost row of PONDER picture and decremented by the vertical print grid increment until desired region of sky plotted.

AZREF = azimuth from radar to grid center column (for case cona $=0$ ).

VARR = magnetic variation.

ATR = azimuth to target relative to radar.

STR = slant range to target relative to radar.

ERT = elevation angle to target relative to radar.

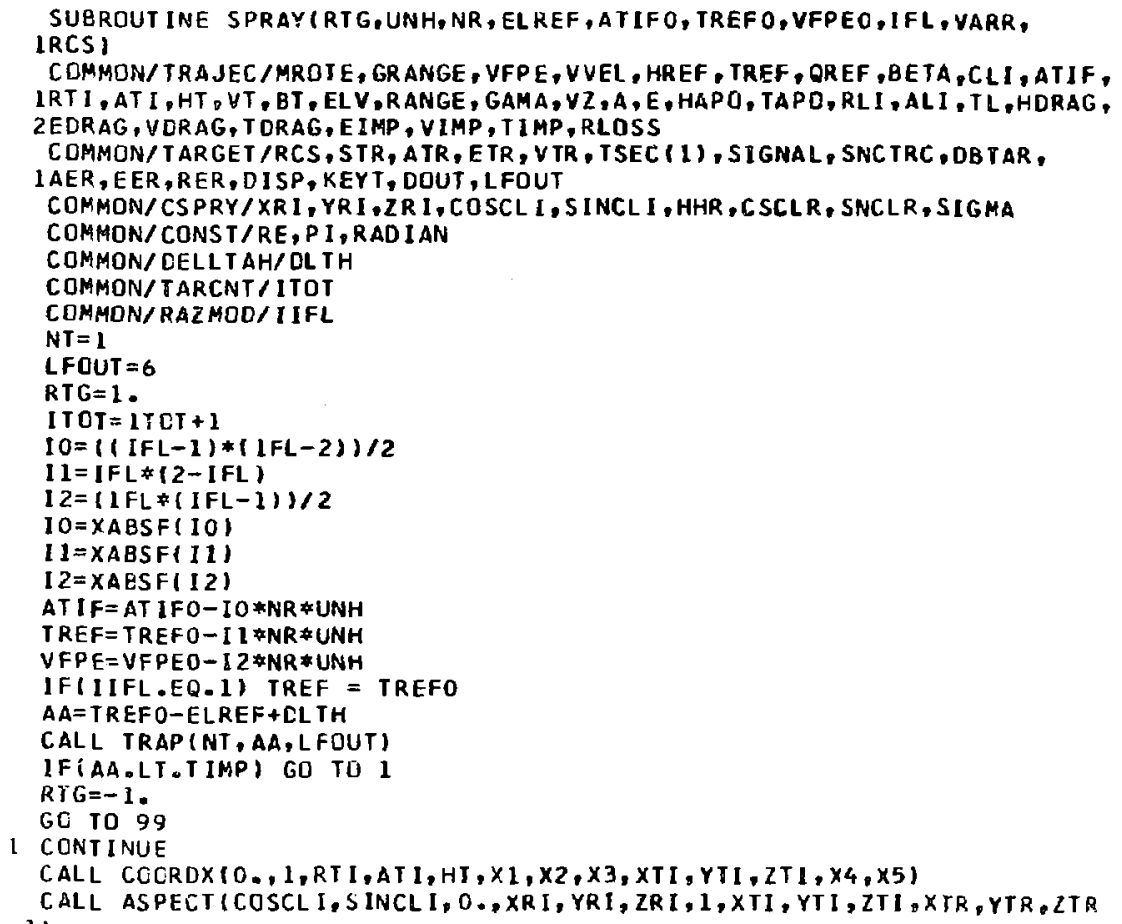




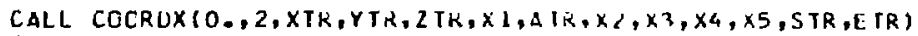

$A T R=A T R-V A R R$

CALL ASPECTICSCLR, SNCLR, HHR, XTK,YIK,ZTR, $1,0,0,0,0, X K T, Y K T, Z R T)$ $X L=V T * C C S F(E L V * R A C I A N)$

$V T R=(X I * S I N F(B T * R A U I A N) * X R T+X I * C U S F(E T * R A L I A N) * Y K T+V T * S I N I$ (tLV* (RAOIANI $\angle L R T$ ISTR

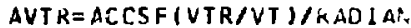

$R C S=R V C S(S I G M A, A V T R)$

99 CONTINUE

RETURN

END

FUNCT ION RVCSISIGMA, AVTR

DIMENSICN RS (30), ASPAI 3C)

DATA RS $/ .005, .005, .0053, .0559, .0071, .0097, .0174,049,0.089,0182$, 1

2

3 $.0605, .05, .0303, .0105, .01, .0091, .0076,000517, .005$. $.005 /$

OATA ASPA $10,40,45, .50, .55,60,165,, 70,72 \ldots, 74 \ldots, 76 \ldots, 78 \ldots, 79 \ldots, 80 \ldots$ $1 \quad 81,85, .90,195 \ldots 94, .100,, 101,102 \ldots, 104, .106 \ldots, 108 \ldots 1$ $2110 \ldots 115 ., 120 \ldots+125 ., 180.1$

IFISIGMA.GT.0.) GE TO I

$I A=2$

$002 \quad 1=2,30$

IF(AVTR.GT.ASPAII) GD TO 2

$I A=I$

$I=30$

2 CONTINUE

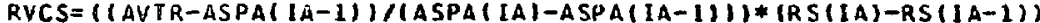

$1+R S(I A-1)$

GC TO 99

1 CONTINUE

$R V C S=S I G M A$

99 RETURA

END 
This is an excerpt from the RANC routine showing the modifications needed to run PALLOT.

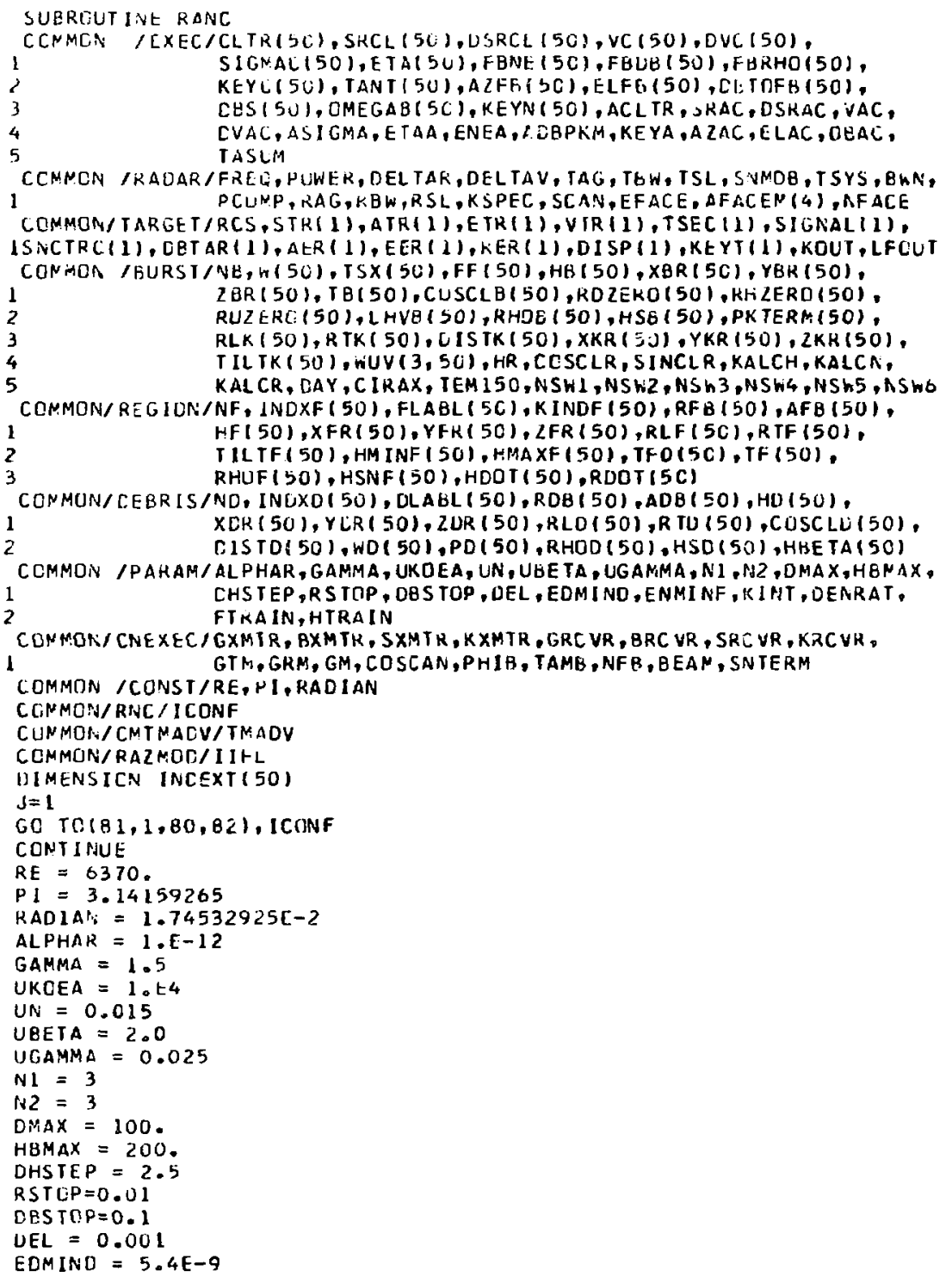


ENMINF $=5 . E_{4}$

KINT.- I

DENRAT $=2$.

FTRAIN=1.

HTRAIN=1.

KASE $=0$

KSPEC $=0$

NFACE $=0$

$\mathrm{NB}=0$

BOLT $T=1.3805 E-20$

$C=2.998 E+5$

$D B C D N=4.34294482$

FOURP I $=4$. \#P!

$T A M B=250$.

HACMIN $=90$.

HACMAX $=130$.

RHOAC $=1 \cdot E-10$

1 EALL RANCIN(KASE)

IF (POWER) 2,730, 2

2 SNMIN $=10 . * \$($ SNMDB $/ 10$.

GXMTR $=10 . *$ (TAG/10.)

$B \times M T R=7$ BW $* R A D$ IAN

SXMTR $=10 . *(-\mathrm{TSL} / 10$.

KXHTR $=$ HOD $(\mathrm{KSPEC}, 100) / 10$

GRCUR $=10 . * *(R A G / 10$.

$B K C V R=R B W \neq R A C I A N$

$\left.S R C \cup R=10_{0} \neq 1-R S L / 10.\right)$

$K R C U R=M O D(K S P E C, 10)$

DEL $R C=1 \circ E-3 * D E L T A R$

DELRU $=D E L R C * P C O M P$

SNT ERM $=P O W E R *(C / F R E Q) * * 2 * P C$ OMP $/($ FOURP $I) * 3$

I F (NB) $11,20,11$

$1100 \quad 12 \quad J=1$, NB

12 COSCLB(I) $=(C O S C L R *(R E+H R+Z B R(I))+S I N C L R * Y B R(I))=(R E+H B(I))$

20 CONT I NUE

IF(TMADV.GT.0.) GC TO 83

¿2 CONTINUE

JT I $M E=\mathbb{I}$

$T$ I $A E=T S E C$ ( JT IME )

CALL PHENOM(TSEC, JTIME)

IFIICDNF.EQ.4) GD TO 80

83 CONTINUE

RETURN

8n COHTINUE

IFIIIFL.NE. II GO TO 9998

$\mathrm{JT} I \mathrm{ME}=\mathbf{I}$

T ( HE = TSEC ( JT IME )

CALL PHENOMITSEC, JTIME)

9998 CONTINUE

T ASUM = T SYS

AZBEAH=ATR ( J)

ELBEAM=ETR( $)$

END 


\section{COORDX}

This routine accepts coordinates of a point in any of five systeme and transforms them to all of the other systems.

Inputs :

HO = altitude or origin of coordinate systems $(\mathrm{km})$.
MODE =
1
2
3
4
5
RXSE = gnd rnge (km) X (east, km) slnt rnge (km)
elev (deg) alnt rnge $(\mathrm{km})$
AYAA = azimuth (deg) $Y$ (north, $\mathrm{km}$ ) azimuth (deg) azimuth (deg) azimuth (deg)
$\mathrm{HZEH}=$ altitude $(\mathrm{km}) \quad Z(\mathrm{up}, \mathrm{km}) \quad$ elev $(\mathrm{deg}) \quad$ altitude $(\mathrm{km})$ altitude $(\mathrm{km})$

Outputs :

$R, A, H=$ surface range, azimuth, altitude coordinates of point.

$X, Y, Z=$ rectangular tangent-plane coordinates of point.

$S, E \quad$ = slant range, elevation coordinates of point.

SUBROUT INE COORCX (HO,MOOE, RXSE, AYAA,HZEH,R,A,H,X, Y, Z,S,E)

COMMON /CONST/RE,PI, RADIAN

$R E H=R E+H O$

GO TOI11,21, 31,41,51), MODE

$51 U 1=(H Z E H+R E) \neq 2+R E \div 2-R \times S E * 2$

$U 2=2$. $* R E \div(H Z E H+R E)$

RXSE=ACCS(UL/U2)

$11 R=R X S E$

$A=A Y A A$

$H=H Z E H$

$U O=R / R E$

$U 2=A * R A C I A N$

$\mathrm{U3}=\mathrm{RE}+\mathrm{H}$

$U I=U 3 \# S$ IN (UO)

$X=U 1 * \operatorname{SIN}(U 2)$

$Y=U 1 * \operatorname{Cos}(U 2)$

$Z=U 3 * \operatorname{COS}(U 0)-R E H$

If IUO.EG.0.1 $Z=\mathrm{h}-\mathrm{hO}$

If $(U 1) 23,25,23$

$21 X=R \times S E$

$Y=A Y A A$

$Z=H Z E H$

$U I=X * 2+Y \neq * 2$

IFIU1) $22,24,22$

$22 \cup 2=R E H \Leftarrow Z$

U3=SQRT $\{U 1+U 2 * 2\}$

$U I=S O R T$ (UI)

$R=A S I N(U 1 / U 3) * R E$

IF (UZ .LT.D.O) R=PI $\nLeftarrow R E-R$

$A=A S I N(X / U I) / R A C I A N$

IF $\left(Y \cdot L T \cdot O_{0} O\right) \quad A=180 .-A$

$H=U 3-R E$

. $3 S=S$ QRT (UI $* 2+Z * 2)$

$E=A S I N(Z / S) / R A D I A N$

GO TO 99

$24 R=0$.

$A=0$.

$\mathrm{H}=\mathrm{HU}+2$ 
$25 S=A B S(Z)$

$E=90$.

IF (Z.LT.0.0) $E=-90$.

GO TO 99

$31 S=K \times S E$

$A=A Y A A$

$E=H Z E H$

U2 $=A * R A C I A N$

$U 3=E * R A C I A N$

$U I=S * \cos \left(U_{3}\right)$

$x=U 1 * \operatorname{SIN}(U 2)$

$Y=u 1 * \cos \left(U_{2}\right)$

$Z=S * \operatorname{Sin}(L 3)$

$U 2=R E H+Z$

U $3=S Q R T(U 1 * 2 * U 2 * * 2)$

$R=A S I N(U 1 / U 3) * R E$

IFIU2.LT.0.0) R=PI*RE-R

$\mathrm{H}=\mathrm{U} 3-\mathrm{RE}$

GO TO 99

$41 E=R \times S E$

$A=A Y A A$

$\mathrm{H}=\mathrm{H} Z \mathrm{EH}$

$U 2=A E R A C I A N$

$U 3=E \otimes R A C I A N$

$\operatorname{CDS} E=\operatorname{COS}(\mathrm{U} 3)$

SINE $=\operatorname{SIN}(U 3)$

$S=H T O S(H O, H, C O S E, S$ (NE)

$U 1=5 \div \operatorname{COSE}$

$x=U 1 \backsim \operatorname{SIN}(U 2)$

$Y=U L * \cos (U 2)$

$Z=S \approx S$ INE

$\mathrm{U} 3=\mathrm{RE}+\mathrm{H}$

$R=A S I N(U L / U 3) * R E$

IF(REH+Z.LT,0.0) R=PI*RE-R

99 KETURN

END 
This subroutine performs horizontal grid corrections as specified by NCRCT. When the blackout regio.: extends past the PONDER picture limits, subroutine CRCT sets in and extends the bounds by NCRCT number of grids to see if it can reach the outer limit of the blackout region.

NMX $=$ (NMXR) number of targets per row to the left of center column.

NPX $=$ (NPXR) number of targets per row to the right of center column.

NCRCT $=$ grid correction factor (in units of print grid increments).

SUEROUT INE CKCTINMXIRD,NPXIRE, NMXO, NPXO,NMX,NPX, INPXRI, IPLDEN,NCRCT $1,1 L C T, \mid R C T, N T L, N T R, N R L, N R R$ )

$N T L=0$

NT $R=0$

IF ( ILCT EQ EQ C) CO TO I

NMXUPRV = NMXO

NMXO=MI NUF (NMXIRU, NMXO+NCRCT I

IF (NMXOPRV.EQ.NAXO) GD TU I

AH $M X=N M X O \neq I P L C E N$

NT L $=(N M \times O-N M X O P R V) * I P L D E N$

$N R L=N M X+1$

1 CCNTINUE

IFIIKCT.EQ.O) GO TO 2

NPXOPRY $=N P X O$

NPXO $=N$ INOF (NPXIRL, NPXO+NCRCT)

IF (NPXOPRV.EQ.NPXO) GO TO 2

$N R R=-P P X$

NPXRI $=$ NPXO \#IPLEEN

NTR $=$ (NPXO-NPXOPRV) I PL UEN

2 CONTIMUE

RETURN

ENC 
This subroutine checks for the horizontal corrections made by subroutine CRCT and prints out the appropriate information concerning each correction.

ELREF = increment in the vertical direction. ELREF is set to the topmost point to be observed and decremented to the lowest point to be observed.

SUBRDUT INE ANALYZE (NTL, NTR, ILCT,IRCT, ELREF)

IF (NTL.EQ.0) GO TO I

IFIILCT.EQ.O) GO TO 3

HRITE OUTPUT TAPE 6,102 , ELREF

GO TO 4

3 CONTINUE

WR1TE DUTPUT TAPE 6,101 , ELREF

4 CONTINUE

IF (NTR.EQ.0) GO TO 2

IFIIRCT .EQ.OS GO TO 5

WRITE OUTPUT TAPE 6,104, ELREF

GO TO 6

5 CONTINUE

WRITE OUTPUT TAPE 6,103 ,ELREF

6 CONTINUE

GO TO 99

1 CONTINUE

IF(ILCT OEQ.1) HRITE OUTPUT TAPE 6,105,ELREF GO TO \&

2 CONTINUE

IFIIRCT,EQ-1) WRITE OUTPUT TAPE 6,106,ELREF

99 CONTINUE

RETURN

101 FORMAT (47HSUCCESSFUL CORRECTION MADE ON LEFT AT ELEVATION,FL0.5)

102 FORMATI49HUNSUCCESSFUL CORRECTION MAOE ON LEFT AT ELEVATION,F10.5)

103 FORMAT(48HSUCCESSFUL CORRECTION MADE ON RIGHT AT ELEVATION,FI0.5)

104 FORMATI5OHUNSUCCESSFUL CORRECTION MADE DN RIGHT AT ELEVATION, (F10.5)

IOS FORMAT IT3HCORRECTION ATTEMPTED DN LEFT BUT STOPPED BY S. G. BCUNDA IRY. ELEVATION IS, F10.5)

106 FORMAT (T4HCORRECTION ATTEMPTED ON RIGHT BUT STOPPED BY S. G. BOUND 1ARY。 ELEVATION IS,F 10.5I EHID 
This subroutine plots blank horizontal lines for the PONDER picture for cases in which no blackout was found at that elevation. FIT defines the boundaries of the print and area grids using the search grid calculations. The flag IRUN, which $s$ witches the executive routine from search to area grid calculations and vice versa, is set in this routine.

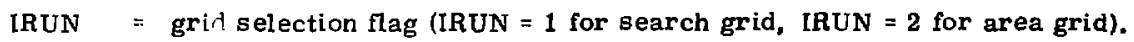
NMXO = number of targets on print grid row to the left of center column. NPXO = number of targets on print grid row to the right of center column. NMX = number of targets on area grid row to the left of center column. NPX = number of targets on area grid to the right of center column. NMXR = number of targets on search grid row to the left of center column. NPXR = number of targets on search grid row to the right of center coiumn. UNITHO = print grid horizontal increment.

UNTVO = print grid vertical increment.

UNITH = area grid horizontal increment.

UNITV = area grid vertical increment.

UNITHR = search grid horizontal increment.

UNITVR = search grid vertical increment.

SUBROUT INE FITINPRES, NLPRES, NRPRES, IRUN, NMXR, NP XR, NMXO,NPXO,NHX, INPX, I PLCEN, IRDEN, MAXNMX, IL, IR)

DIMENSION S(150)

NPREV $=$ NPRES

NLPREV $=$ NLPRES

NRPREV $=$ NRPRES

IFIIL $13,4,3$

4 NPRES $=1$

IF INPREV-215,6,5

6 NMXO $=$ NL PREV $*$ IRDEN

NPXO=NRPREV *IRDEN

NMX $=N M X 0 * I P L O E N$

NPX $=$ NPXO* I PLDEN

I RUN $=\mathbf{2}$

RETURN

5 DO $13 \quad I=1$, IRDEN

IO $=\operatorname{MOO}(1$, IRDEN $)$

IF ( 10$) 14,15,14$

$14 \mathrm{~S}(1)=1 \mathrm{H}$

WRITE $\{8,3001\}$ S(1)

HRITE $(9,3001)$ S(1)

HRITE $\{10,3001\} S(1)$

HRITE(11.3001) S(I)

GO TD 13

$15 J 0=\operatorname{MAXNMX}+1$

DO $16 \quad J=1, J 0$

(6) $S(J)=1 H$

$J l=J 0+l$

$S(J I)=I H-$ 
HRITE $(8,3001)(S(J), J=1, j 1)$

WRITE $(9,3001)(S(J), J=I, J I)$

WRITE $(10,3001)$ (S(J),J $=1, J 1)$

WRITE $(11,3001)(S(J), J=1, J 1)$

13 CONTINUE

3001 FORMAT $(120 A 1)$

$N M X=N M X R$

$N P X=N P X R$

I $R \cup N=1$

RFIURR

3 IF (NPRESI11,12,11

12 WRITE OUTPUT TAPE 6,3009

3009 FORMAT(2BH VERTICAL ESTIMATE TUO SMALL)

11 NPRES $=2$

$N L=N M X R+2-I L$

$N R=N P X R+2-1 R$

NLPRES = MINDF (NL, NMXR)

NRPRES = NINOF (NR, NPXR)

I FINPREV-2 19,10,9

$\checkmark N M X O=N L P R E S * I R O E N$

$N P X 0=N R P R E S * I R D E N$

$N M X=N M X O \#$ I PL CEN

NPX $=N F X O \div$ IPLOEN

$1 \mathrm{KUN}=2$

RE TURN

10 NMXO=MAXOF (NLPREV, NLPRESI*IRDEN NPXO=MAXOF (NRPREV, NRPRES ) * IRDEN

$N M X=N M X O \div I P L D E N$

IPX $=N P X O \approx I P L D E N$

I RUN $=$ ?

RETURA

END 
This subroutine reduces the number KEYT to one digit representing its most significant digit. It then returns this digit to the plotting subroutine for appropriate action.

KEYT = number generated by RANC for target presently being observed (target calculation code).

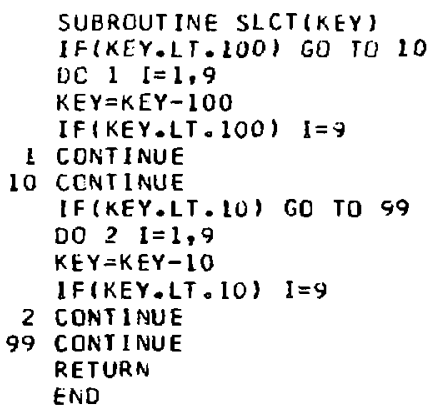




\section{PLOTSN}

This is a plotting subroutine. It takes the signal/noise calculations produced elsewhere, codes them alphanumertcally, point by point. Ind stores them in a row matrix, left to right, for one $r(w$. The coded line is next written into output file PLOTSN.

SIGNAL = number generated by RANC for target presently being observed (this is the completed signal/noise ratio).

KEYT = number generated by RANC for target presently being observed (target calculation code).

BOCR = signal/noise increment between output contours.

NPX = number of targets per row in unrotated system to the right of center column.

NMX = number of targets per row in unrotated system to the left of center column.

NPY = num'ser of rows in unrotated system above the row containing reference peint.

$\mathrm{NMY}$ = numiver of rows in unrotated system belew the row containing reference point.

SUBROUT INE PLOTSNISIGNAL,KEYT, BOCR, DBOCR, NPX, NMX, NPY, NMY, MAXNMX, IP ILDEN, KT APEI

DIMENSIGN SIGNAL $(2), K E Y T(2), R(250)$

$N O=$ MAXNMX $-N M X+1$

$N Y=$ HAXOF $(1, N O)$

$J I=N P Y+N A Y+1$

$K L=N P X+? \cdot M X+1$

$K 2=N 1+K 1$

$I=1$

DD $103.6 \mathrm{~J}=1, \mathrm{J1}$

101 . $R(N)=1 H$

DO $1017 \mathrm{~N}=1, \mathrm{~N} 1$

$001015 K=1, K 1$

$\mathrm{N}=\mathrm{N} I+K$

SIG =SIGNAL (I)-BOCR

IF(SIG.LT.500.) GO TO 1903

$R(N)=1 H *$

GO TO 1015

1903 SONTINUE

I I I I G I 1001,1002,1002

$1002 R(N)=1 H$

GO TO 1015

1001 IF (S IG+CBOCR) 1003,1004, 1004

$1004 R(N)=1 H$.

GO TO 1015

1003 If (SIG+2,*DEOCR) $1005,1006,1006$

$1006 R(N)=1 H C$

GO TO 1015

2005 IF(SIG+3.\#DBOCR ) 1007,1008, 1008

$1008 K(N)=1 H \theta$

Gก TO 1015

1007 If(SIG+4.*DBOCR $1009,1010,1010$

$1010 R(N)=1 H A$

GO TO 1015 


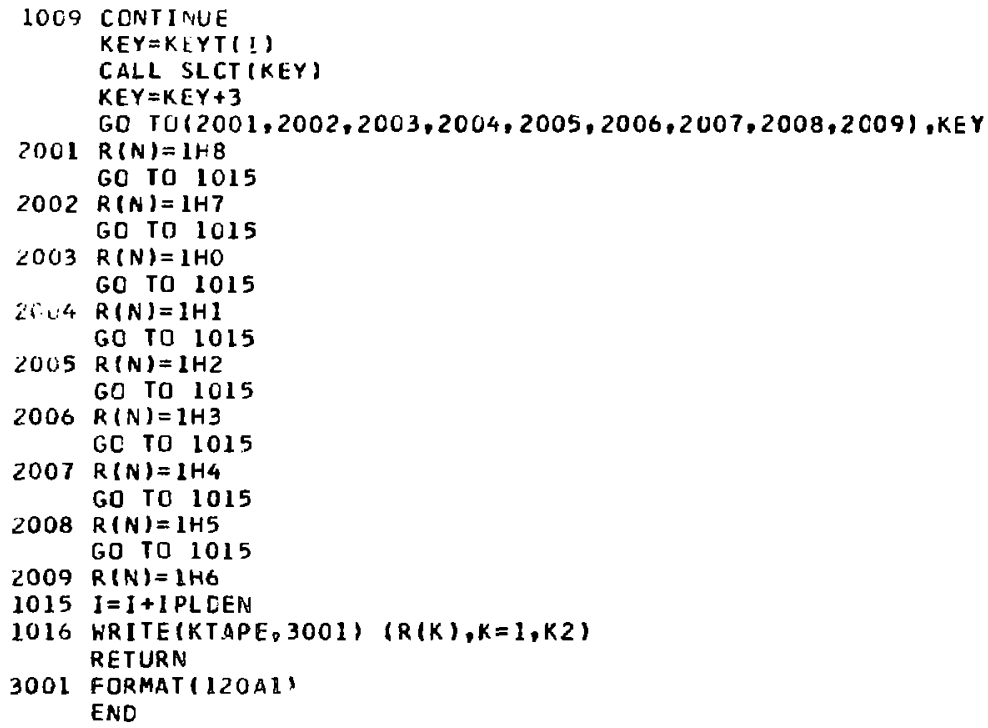


This subroutine computes target position and velocity as a function of time for an elliptical ballistic trajectory over a spherical rotating earth. Keplerian motion is assumed with transformations made to a rotating coordinate system. If atmospheric drag becomes important on the terminal portion of the trajectory, computations are transferred to subroutine RVDRAG and earth rotation is ignored.

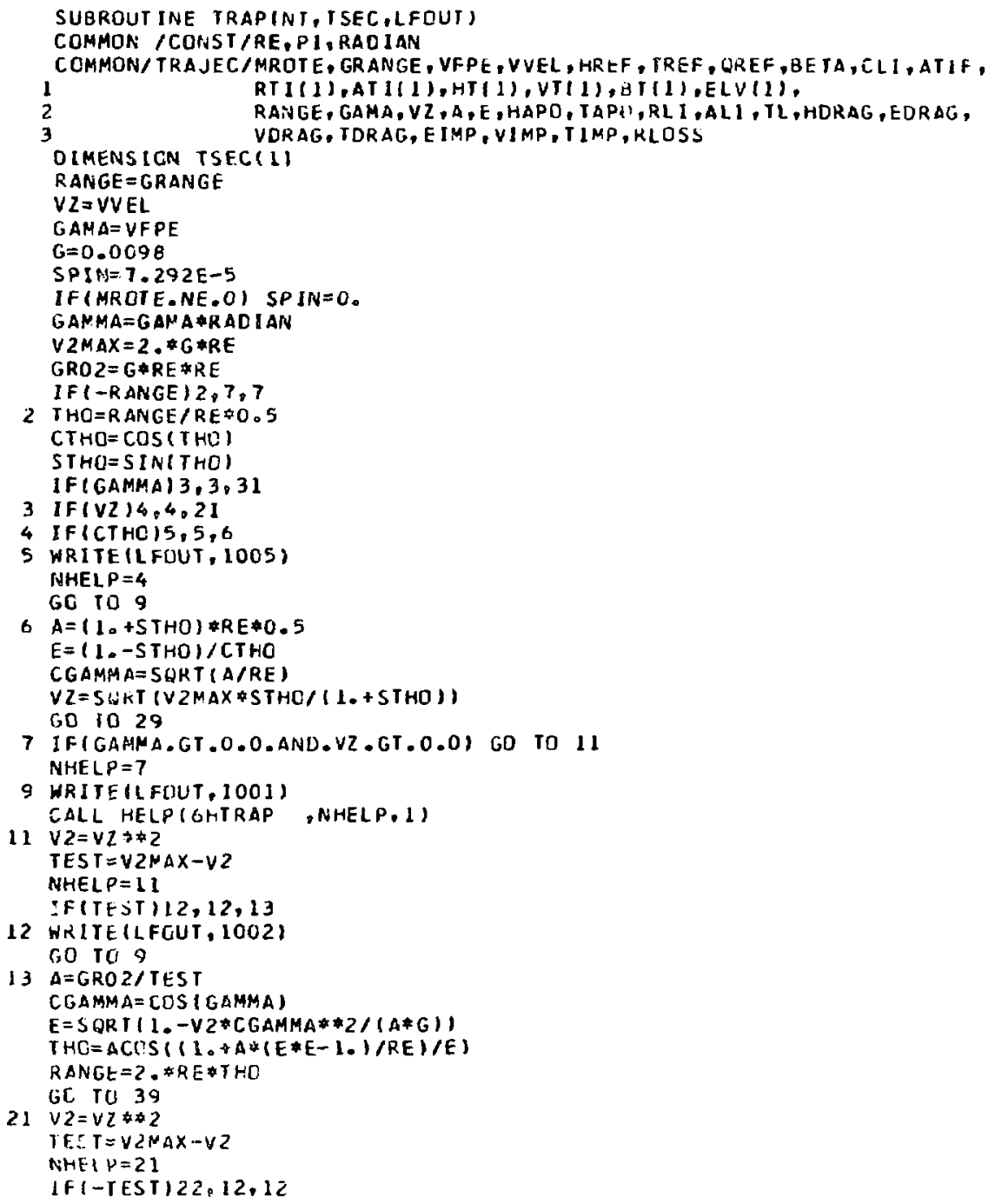


22 It $(V Z-V 2 M A X+5 T+C / 11 .+5 T H) 123,24.24$

23 WKITE LLFCUT, 1003 !

NHELP $=22$

CiC TD 9

¿4 $A=6 R 02 / T$ T.ST

TIST=SGRT $\{(\{R E / A) \neq(\{N D \neq(i .5) \neq 2+1 .-\{R E / A\})$

IF (GANNAI25,28,25

25 IF $(0.5 * V 2 M A X-V 2) 20,20,27$

20 HRITE(LFCUT, 1006$)$

NHELP $=25$

GC TC 9

27 TEST $=-T E S T$

$28 E=0.5 \div R E / A \neq C T H O+T E S T$

CGAMMA $=S O R T(A \# G *(1 .-E * E) / V 2)$

29 G $\triangle M M A=A C O S$ (CGANMA)

GO IL 39

31 CGAMMA $=\operatorname{COS}$ (G $\triangle M M A)$

$V Z=G * R E * S T H O /(C G A M M A * S I N(T H O+G A M M A))$

TEST $=V 2 \mu A X-V 2$

IFITEST) $32,32,33$

32 WKITE (LFOUT, 1004)

NHE LP $=31$

GO TO 9

$33 A=$ GRO 2/TEST

$E=S O R T(2,-V 2 \notin C G A M M A * 2 /(A \star G))$

$V Z=S Q R T(V Z)$

$39 \quad A E=A \div E$

$A E Z=A-A E * E$

$V C O N=S G R T(\triangle A Z Z G R \cup 2)$

$E C C=A C O S((R E-A) / A E)$

$C l=A / R E \$ S Q R T(A / G)$

I $A P C=C 1 *(E C C+E \# S \perp$ iv $(E C C)$ )

$H A P C=A+A E-R E$

GAMA $=$ GANMA'RACIAN

HKE $\bar{r}$ i = AMAX 1 ( AMINI ( HREF, HAPD ), O.)

HORAG $=5 . * A I N T(-7.3 * A L O G(1 . E-3 * B E T A * S I N(G A M M A) / V Z * 2) / 5 .+0.5)$

HDRAG = AMAX $1($ AMIN $1($ HDRAG, HAPO, 120.$), 0$.

ZORAG $=R E+H O R A G$

$E C C=A C C S(12 C R A G-A) / A E)$

$T D K A G=T A P D+C I *(E C C+E+S 1 N(E C C))$

VDRAGI $=$ SGRT $(G R 02 *(2 . / 2$ CRAG-1./A) $)$

EORAG I = ACOS (VCON/ZDRAG/VORAGI)

$R D R A G=R E *(T H D-A C O S((Z D R A G-A E 2) /(Z D R A G * E)))$

COSCLI $=\operatorname{COS}(\mathrm{CL}$ I)

SINCLI = SIN(CLI)

$X I=A T$ IF $\#$ RACIAN

$\cos A D 1=\cos (\times 1)$

SINADI=SIN $(\times 1)$

ROI = RDRAG

$J=1$

$41 \times 1=R D 1 / R E$

$\operatorname{COS} R=\cos (x 1)$

$S I N R=\operatorname{SIN}(X 1)$

C OSCL $L=C D S C L I * C O S R+S I N C L I * S I N R * C O S A D I$

S INCL D=SGRT $11 .-C U S C L[* * 2\}$

CBORAG $=(C O S C L I * S I N R-S I N C L I * C O S K * C U S A B I) / S I N C L D$

SBCRAG $=-$ S INACI\#SINCL I/S INCLD

$B D R A G=A S I N(S B C R A G)$

IF (CBCR AG.LT.0.) CDRAG=PI - BDRAG

$\checkmark A T$ MOS $=$ SPIN ?. DRAG $\$$ INCLD

$K 1=V O R A G I * C O S(E C R A G I)$

BDKAGI = EDRAG+ASINIVATMOS $\$ C B D K A G / X I)$ 


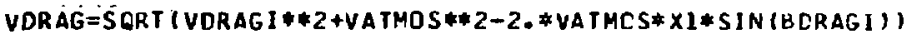
EDRAG = AS IN (VDRAG I $\$ S$ IN( EDRAG I) / VDRAG)

IF ( HREF 1-HDRAG $51,52,52$

51 IF (OREF $155,52,55$

$52 \mathrm{ECC}=A \mathrm{COS}($ (RE+HREF $1-A) / A E)$

$T D E L=C I *(E C C+E * S I N(E C C))$

IFIOREF.EQ.0.1 TUEL=-TDEL

TDEL $=T A P O+T D E L$

TI=TDRAG

$H I=H O R A G$

VI \pm VDRAG

$F 1=$ EDRAG

$K I=R D I$

GO TO 56

55 TOEL $=1 . E 10$

CALL KVORAGIG, BETA,TDRAG, HDRAG, VORAG, EDRAG,RDI, TDEL, HREFI, VI, EI,

IRII

I I= TDEL

$H I=H R E F 1$

56 TL=TREF-T DEL

$T I=T I+T L$

$T$ I $M P=1 . E \perp O$

$H F=0.0$

CALL RVORAG (G, BETA,TI,HI,VI,EI,RI, TIMP,HF, VIHP, EIMP,RLOSS)

IF 10.001 -ABS(RLOSS) 57.58 .58

57 RDI =RDI-RLDSS

$J=J+1$

If (J.GT.10) CALL HELP(6HTRAP .57.1)

60 TO 41

58 TAFO=TAPO+TL

TDRAG $=$ T CR AG $\$ T L$

RLOSS =RCRAG-RDJ

CALL COOROX $10,, 1, R 01, A T I F, H D R A G, X 1, \times 2, \times 3, \times 01, Y 01,20 I, \times 4, \times 51$

CALL ASPECT (COSCLI,SINCLI, $0 \ldots, X 0 I, Y D I, 20 I, 1,0, \ldots \ldots, 0 \ldots X I 0, Y[0,210)$

ATD = ( BDRAGI-PI) \&RADIAN

$T P=T L$

$1=0$

IFINT $160,95,60$

$60 I=I+I$

$T I=\operatorname{TSEC}(1)$

$61 E M=(T I-T A P O) / C l$

IFITI-TL $62,62,63$

$622 I=R E$

$\mathrm{HI}=0.0$

$\mathbf{V} \mathbf{I}=\mathbf{V} \boldsymbol{Z}$

$E I=G A M H A$

AI $=$ RANGE

GR TO 68

63 IF(TI-T DRAG) $64,64,8 \mathrm{C}$

64 ECC $=E M$

$65 \mathrm{FCCl}=\mathrm{ECC}$

$E M I=E C C 1+F * S I N(E C C L)$

$E C C=E C C 1+(E M-E M 1) /(1++E * C O S(E C C 1))$

IF (ABS ( ECC-ECC 1 )-1 - E-6*ABS ( ECC ) $166,66,65$

$66 \quad L I=A *(1 \cdot+E * \operatorname{COS}(E C C))$

$H I=I I-R E$

$V I=\$ 4+1 ;(S R 02 *(2 . / 2 I-1 . / A))$

$E I=A C O S(Y C O N / Z I / V I)$

$R J=R E *(T H D-S I G N(A C D S(I Z I-A E Z I /(Z I * E)), E H))$

68 RTD $R_{1}-R D R A G$

CALL COORDXIHDRAG, I, RTD,ATD,HI, XI, X2,X3,XTD,YTU, $2 T 0, \times 4, \times 5 I$

CALL ASPECTICOSCLD,SINCLD, HDRAG,XID,YID,ZID,,$X$ TD,YYD, ZTD,XII, YTI, 
$12 T(1)$

ULGNG = SPIN $(T C R A G-A M A X I(T), T L)$

$X I=A S I N I S I N C L I * S I N(0.5 *$ DLONGI)

AI I =-AS IN (CCSS $(0.5 \% D L O N G) / C O S(X !)) / R A D I A N$

IFICOSCLI.LT.0.I AII=180.-AII

RII $=2$ \#RE $¥ X I$

CALL CCCRDX $(0,1,1, R 11, A 11,0,, \times 1, \times 2, \times 3, \times I I, Y 1 I, Z 1 I, \times 4, \times 5)$

CALL ASPECT ICOSCLI, SINCLI, 0.,XII,YII,ZII, I, XTI, YTI,ZTI, XII, YTI,

(2TI)

CALL COORDX $(0,1,2, X T I, Y T I, Z T I, R I, A I, \times 1, \times 2, \times 3, \times 4, \times 5, \times 6)$

IF(IL-TI) TI,71,87

71 CDSA $=-$ CCS (BDRAGI)

$X I=R T D / R E$

$\operatorname{COS} R=\cos (\times 1)$

$S I N R=S I N(X 1)$

COSCLT $=$ COSCLD*COSR+S INCLD*S INR *CDSA

SINCL T $=$ SQRT $(1 .-C O S C L T * 2)$

$\operatorname{COS} B=($ COS CLD*S INR-S INCL D*COSR *COSA)/SINCL T

SINB = SINCLD*S IN(BCR AGI I/SINCL T

VATMOS $=S P I N * Z I * S I N C L T$

$X I=V I * \operatorname{CCS}(E I)$

VTI $=S G R T(V I * 2+V A T M O S * 2-2$ * *VATMOS*X1*SINB)

ETI=ASINIVI*SIN\{EIS/UTI\}

$X 2=V T I * \operatorname{COS}(E T I)$

SINBT I $=(X I$ *SINB-VATMOS $) / X 2$

$\operatorname{CCSB} T 1=X 1 * \operatorname{Cos} 8 / \times 2$

GO TO 91

80 I $F(T I-T$ IMP) $8 \mathbb{1}, 81,86$

$81 \mathrm{HF}=0.0$

IFITP-TLRAG $182,82,83$

82

$T P=T D R A G$

$H I=H D R A G$

VTI = VURAG

$E T I=E D R A G$

$R I=R D I$

83 CALL RUCRAG (G, BETA,TP,HI,VTI,ETI,RI,TI,HF, VTI,EII,RL)

$H I=H F$

$T P=T I$

$A I=A T I F$

$X I=R I / R E$

$\operatorname{COS} R=\cos \left(x_{1}\right)$

$S$ INR $=$ SINIX::

COSCL. $T=C O S C L I * C O S R+S I N C L I * S I N R * C O S A D$

SINCL. T=SQRT $(1,-C O S C L T * * 2)$

COSBT $I=(\operatorname{COSCL} I * S I N R-S I N C L I * \operatorname{COSR} * \operatorname{COSADI}) / S I N C L T$

$S$ INBT I $=-S$ INADI*SINCL I $/ S$ INCL T

GO TO 91

86

$R 1=0.0$

$A I=0.0$

$H I=0.0$

87 VT I $=0.0$

$S$ INBT $I=0.0$

$\operatorname{COSBT} 1=1.0$

ET I $=0.0$

91 IF I I-NT $) 92,92,96$

$92 \operatorname{RTI}(I)=R I$

ATI $(I)=A I$

$H T(I)=H I$

$V T(I)=V T I$

BT ( I ) = ASIN ISINBT I )/RADI AN

IF(COSBTI LLT_0.) BT (I) $=180,-B T$ (I)

ELV I $)=S I G N(E T I,-E M) / R A D I A N$ 
I F ( T I -T IMP ) 93,94, 44

93 IF (I-NT) $60,95,60$

$94 \mathrm{NT}=\mathrm{I}$

$95 \quad I=N T+1$

$\mathrm{TI}=\mathrm{TL}$

GC TO 61

$96 \quad R L I=R I$

$A L I=A I$

EDRAG $=-E D R A G / R A C I A N$

$E I M P=-E I M P / R A C I A N$

RETURN

LUOI FORMATI28HOTP.AJECTORY GATA INPUT ERRORI

1002 FORMATIGOHOLAUNCH VELOCITY EXCEEOS ESCAPE VELOCITYI

1003 FORMAT (5OHOL AUNCH VELOCITY LESS THAN MINIMUM-ENERGY VELOCITY)

1004 FORMAT (27HOLAUNCH ELEVATION TOL LARGE)

1005 FORMATI 89 HOM INIMUM-ENERGY TRAJECTORY REQUESTED FOR SURFACE RANGE E IXCEED ING HALF EARTH CIRCUMFERENCEI

1006 FORMAT (33HODEPRESSED TRAJECTORY INTERSECTS EARTH)

END 


\section{PLOTC}

This is anuther plotting subroutine. It takes the clutter jnoise calculations produced elsewhere, codes them alphanumerically, point by point, and stores them in a sow matrix, left to right, for one row. The coded line is next written into output file PLOTC.

CLTR = number generated by RANC for the target presently being observed (this is the completed clutter/noise ratio).

KEYT = number generated by RANC for target presently being observed (target calculation code).

BOCR clutter/noise increment between output contours.

NPX = number of targets per row in unrotated system to the right of center column. $\mathrm{NMX}=$ number of targets per row in unrotated system to the left of center column. NPY = number of rows in unrotated system above the row containing reference point. NMY $=$ rumber of rows in unrotated system above the row containing reference pount.

SUBROUT INE PLOTCICLTK, KEYT, BOCE,DBOCR, NPX, NMX,NPY,NMY,MAXNMX,

I I PLDEN, KT APE)

UIMENSICN CLTR(2),KEYT(2),R(150)

NO $=M A X N M X-N M X+1$

$N 1=M A X O F(1, N O)$

$J 1=N P Y+N N Y+1$

$K 1=N P X+R M X+1$

$K 2=N 1+K 1$

$1=1$

$001016 \mathrm{~J}=1, \mathrm{Jl}$

DO $1017 \mathrm{~N}=1, \mathrm{~N} 2$

$1017 R\{N\}=1 H$

DO $1015 K=1, K 1$

$\mathrm{N}=\mathrm{N} 1+\mathrm{K}$

$C L=C L T R(l)-B O C R$

(FICL.GT.(-500.)) GD TD 1904

$R(N)=1 H$

GO $\quad 1015$

1904 CUNI, UE

IF (CL.GE.0.) GG TO 1001

$R(N)=1 H$

GD TG 1015

IOUL IFt(CL-CBOCR).GE.U.) GO TO 1002

$R(N)=1 H$.

GU TD 1015

1002 If ( (CL-2. $[B] C R) . G E .0$.$) GO TO 1003$

$R(N)=1 H I$

GO IO 1015

1003 IF( (CL-3. $\$$ CBOCR ). EE.0.) G() TU 1004

$R(N)=I H H$

GO TO 1015

1004 IF( (CL-4. \#[BOCR).EE.U.) GO TO 1005

$R(N)=1 H G$

GC TC 1015

1OU5 [F(ICL-5. \#CBCCR).GE.U.) GO TO $100 t$

$R(N)=:$ i $F$

GO TO 1015 


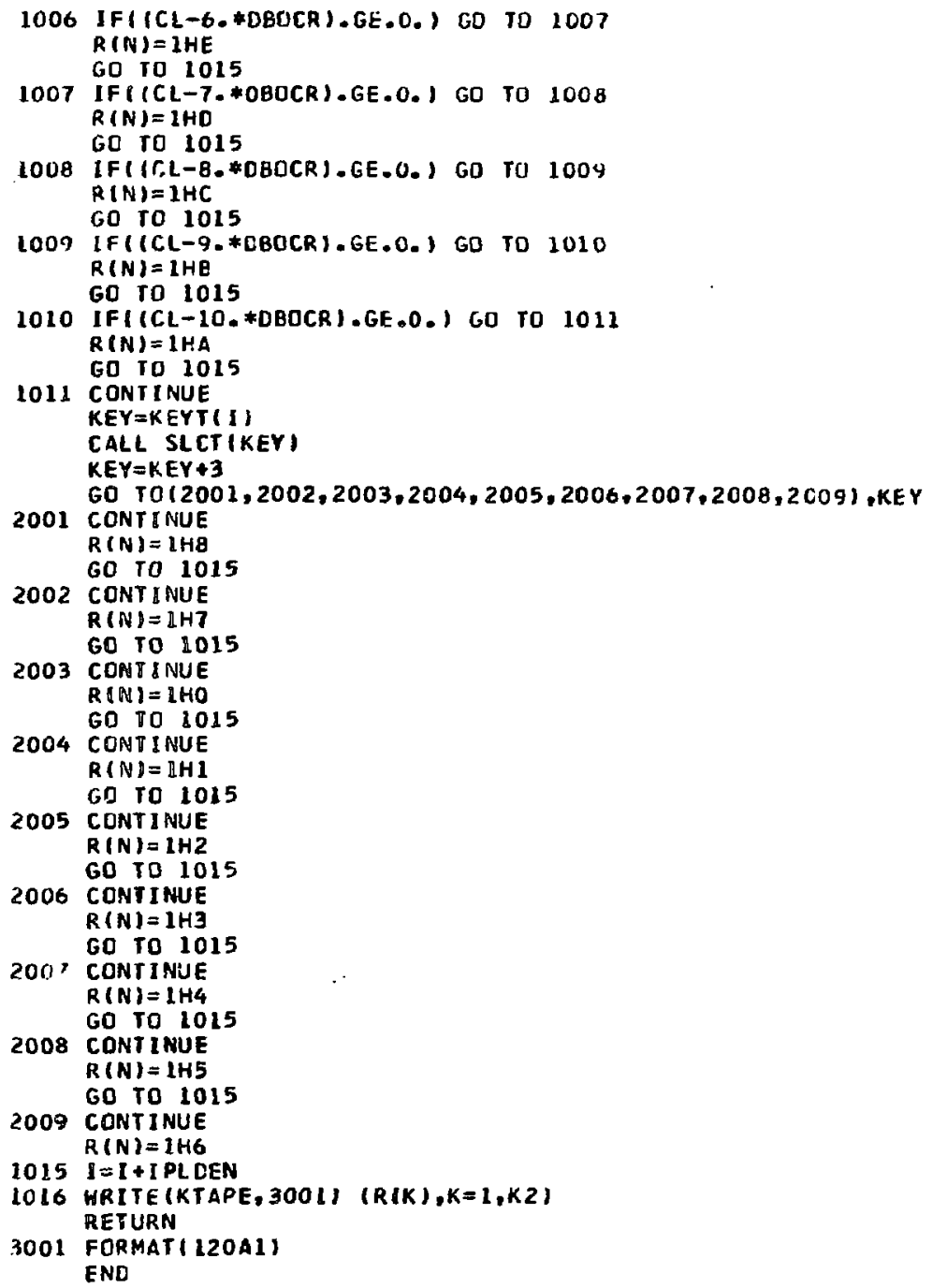




\section{AREA}

This subroutine calculates the area of radar blackout on the target surface.

SUEROUT INE AREAIS IGNAL, SSIGNAL, BOCR I T, UNI TH, UNI IV, NMX, NPX, NT, MAXNT 1. I AREA, AREA I, AREAC)

DIMENSICN SIGNAL ( 2$)$, SS I GNAL ( 2$)$

$N T P 1=N T+1$

MXNTP $1=$ MAXNT +1

DQ $100 \quad I=1$, NT

IF (SIGNAL (I)-BOCRIT) 101,102,102

IOI SIGNAL (I) $=1$.

GO TO 100

$102 S[G N A L \mid 1)=0$.

100 CONIINUE

LO $103 I=N T P 1, M \times N I P I$

103 SIGNAL $(I)=0$.

IF(IAREA) I,2。]

2 NMXPREV $=$ NMX NPXPREV $=N P X$

1 I GAP $=N M X-N M X P R E V$

IFIIGAP I10,13,12

12 DO $201=1$,NTPREV

20 SS I GNAL (NTPREV+ IGAP+1-I) I=SS I GNAL (NTPREV+1-1)

D0 $30 I=1$, I GAP

30 SSIGNAL $(I)=0$.

13 ISHIF T $=0$

GO TO 11

10 I GAP $=-1$ IGAP

DO $40 \quad 1=1$, NT

40 SI GNAL (NT + IGAP+I-I) =SIGNAL (NT+I-I)

DQ $50 \quad 1=1$, IGAP

50 SIGNAL (I) $=0$.

I SHIF T = I GAP

$11 N M=M A X O F$ (NHX,NMXPREV ? +MAXOF (NPX,NPXPREV)

$N M F 2=N M+2$

DO $\quad 120 \quad I=1, N M$

NO $=$ SI GNAL $(I)+5$ I GNAL $(I+1)+5 S I G N A L I I)+S S I G N A L(1)+1$.

GO TO(120,121,121,121,122), NO

121 I FL $A G=1$

GO 10123

122 IFLACi=2

123 CONTINUE

$A=$ UNI TV *UNITH

GJ TO $\{127,128\}$, IFLAG

128 AREAI = AREAI+A

127 AREAD $=A R E A D+A$

120 CONTINUE

NTPREV $=N T$

NMXPREV $=$ NMX

NPXPREV $=N P X$

I AREA $=1$

DO $60 \quad 1=1$, NT

60 SSIGNAL (I) =SIGNAL (ISHIFT+I)

DO $70 I=N T P 1, N H P 2$

70 SSIGNAL $(I)=0$.

RETURN

END 


\section{RNCOT}

This subroutine is documented in the RANC IVA manual. (See Ref. $f$, of this document )

SUBROUT INE RNCGT

END 


\section{Appendix 2}

\section{Description of PALLOT Input Cards 5, 7, 8, and 9}

This description will deal first with the impant point location parameters (fields 6 and 7 of card 5), and then the four modified cards $(7,8,9,9 a)$ of the input package will be described. (Refor to Fig. 4.) 


\section{CARD 5}

FORMAT (I1, E9.0, 610.0)

NCARD = card identifier (equals five).

$X(S)=$ ground range from origin to impact point.

$X(7)=$ azimuth from origin to impact point. 


\section{CARD 7}

FORMAT \{I1, E9.0, 6E10.0, 1YI1 $\}$

NCARD = card identifier (equals seven).

TSEC (NT) = RANC calculation time relative to burst (NT).

MAXNMX = number of columns to the left of the center column on the printout (horizontal translation of the picture).

NCRCT = print grid correction (zero disables) for blackout projections on the PONDER picture; i.e., used to try and encompasa complete blackout area by extending the print area at the appropriats portions of the PONDE:R picture.

SIGMA = radar cross-section (meters square).

MROTE = term for the earth's rotation:

$0=$ yes, use model with rotating earth.

$1=$ no, don't use model with rotating earth.

QREF = trajectory reference:

1 = use descent portion of trajectory.

$0=$ use assent portion of trajectory.

DLTH = correction factor needed to place print grid (vertical) increment exactly on the burst trajectory intersection point.

IFL $\quad=$ mode switch:

\begin{tabular}{clc}
\hline IF L value & PALLOT mode & Increment \\
\hline 0 & azimuth & ATIF \\
1 & inline like & TREF \\
2 & elevation & VF PE \\
3 & complete time & UNITV0 \\
& histciy & \\
\hline
\end{tabular}




\section{CARD 8}

FORMAT ([1, E9.0, 6E10.0, 1XI1)

NCARD = card identifier (equals eight).

ATIFO = the reference azimuth of the RV's trajectory to be calculated (deg).

GRANGE = the ground range between the RV's launch point and its impact point (km).

VFPEO = The RV's launch angle (deg).

VVEL = the RV's launch velocity $(\mathrm{km} / \mathrm{sec})$.

HREF = the reference altitude at which the burst and the reference trajectory intersect $(\mathrm{km})$.

TREFO = the reference time that the $R V$ intersects the burst's center; relative to burst time (sec).

BETA = ballistic drag coefficient for $\mathrm{RV}\left(\mathrm{lb} / \mathrm{ft}^{2}\right)$.

TMADV = time advance switch:

$0=$ time constant over PALLOT surface.

1 = time change over PALLOT surface. 
CARD 9

FORMAT (I1, E9.0, 6E:0.0)

NCARD = card identifier (equals seven).

PLDEN = ratio print grid increments/area grid increments.

RDEN = ratio search grid increments/area grid increments.

UNITHO = print grid increment (depends on mode of operation) in horizontal direction.

UNITVO = print grid increment in vertical direction (sec).

NMX0 = the number of columns to the left of the center column, where the center column of the plane is located by the normal line at the point of tangency.

NPX0 = the number of columns to the right of the center column.

NFIR = this variable is lacated in columns 66 through 70. NHR is the total number of seconds from the earth's surface to the topmost point to be observed on the RV's trajectory. NHR is specified in units of UNITVO (i.e., INHR $=20$ weans that 2 is topmost point $=20$ times UNTVO sec to the topmost point of the PALIOT picture).

NHR1 = this variable is located in cclumns 61-65. NHR1 is the total number of seconds to be covered in the PALLOT picture, from the topmost point (NHR) to the bottom of the picture. NHRI is also given in terms of UNITV0 and, therefore, designates the number of print rows that will compose the PALLOT picture. If NHR 1 is set to zero or blank, the program automatically sets NHR1 equal to NHR, causing NHR number of print rows. 
CARD 9A

FORMAT (I1, E9.0, 6E10.0, 1 XI1, 2XA6)

NCARD = card identifier (equals eight).

SBOCRIT = maximum threshold of signal/noise and of signal/(noise plus clutter) (in $\mathrm{dB}$ ) for radar recognition.

DSNBOCR = the separation (in $\partial B$ ) between the final PONDER picture signal/noise and signal /(noise + clutter) contours.

THCLTR = minimum threshold for clutter (in $d B$ ) for radar recognition.

DTHCLTR = the separation between the final PONDER picture clutter contours.

THDLFF = the maximum threshold for signal/notse minus clutter/noise for radar recognition.

CONA $=s$ witch that signifies that card is to be read as card nine (CONA $=0$ ) or as carci nine A (CONA = 1). 


\section{Appendix 3 \\ Examples of $\mathrm{OZ}$ and TY Overlays}

EXAM PLE OF OZ OVERLAY OPERATING ON A PALLOT AZIMUTH MODE RUN

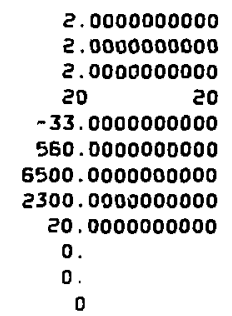

2.0000000000

. 0000000000

2. 0000000000

$-33.0000000000$

560.0000000000

6500.0000000000

300.0000000000

0 .

0

\author{
3.0000000000 \\ 3.0000000000 \\ 3. 0000000000 \\ $70 \quad 70$ \\ 1. 0000000000 \\ 600.0000000000 \\ 0 . \\ 1. 5194364475 \\ 5.0000000000 \\ 0 . \\ o.
}

20

1.0000000000

0 . 


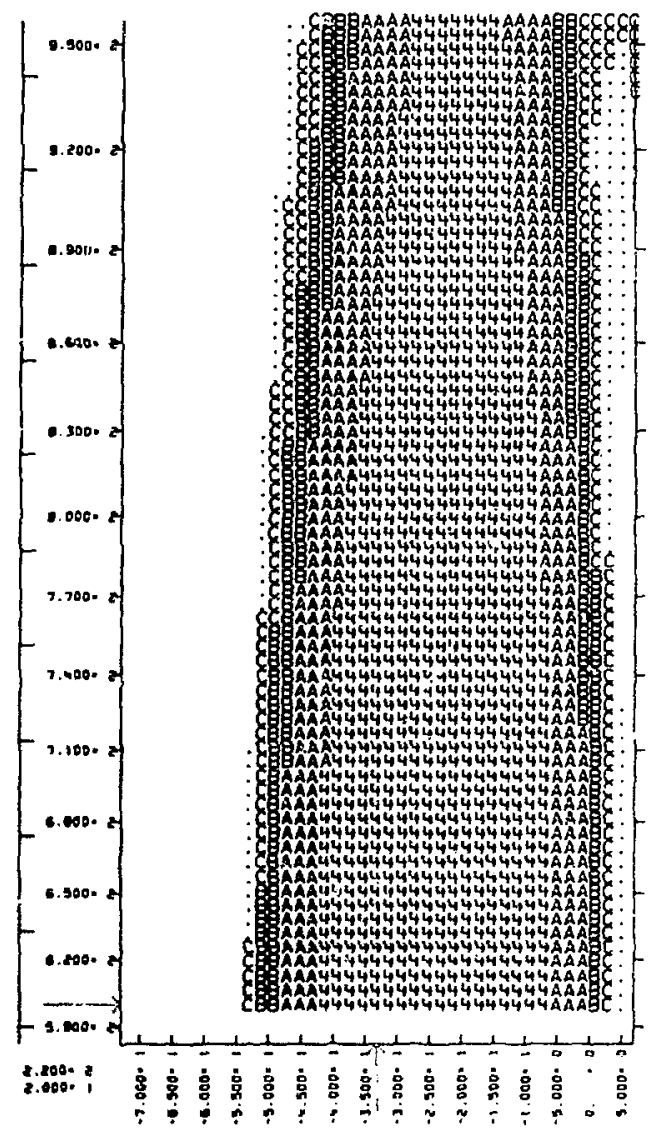




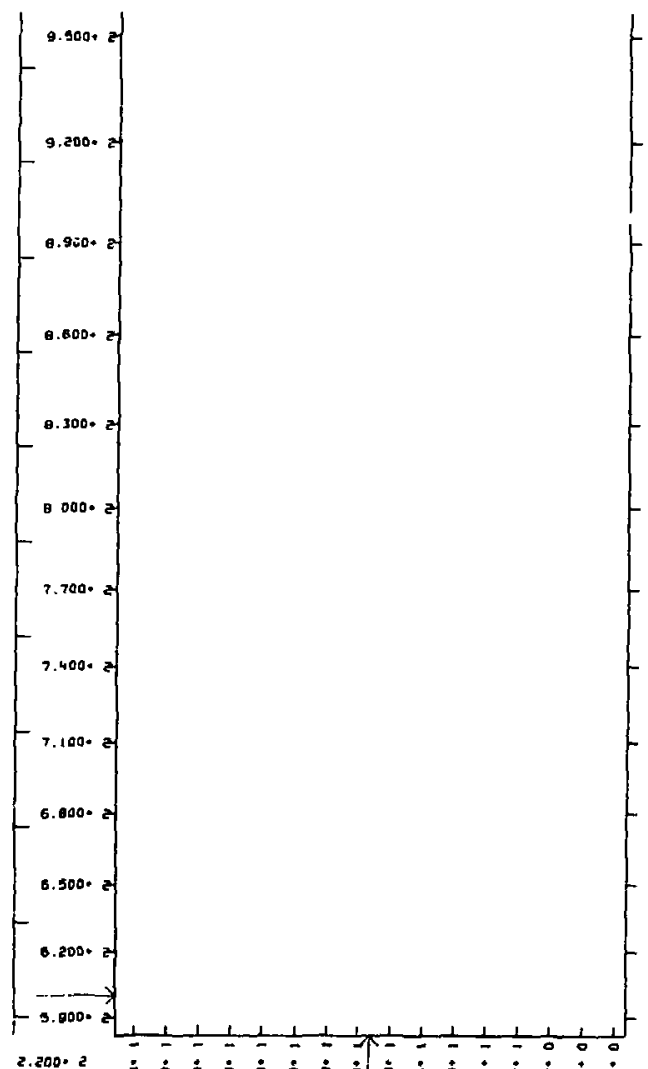

$2.200 \cdot 2$
$2.000 \cdot 1$

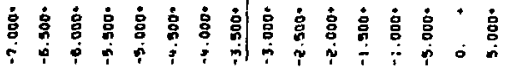

$-57-$ 


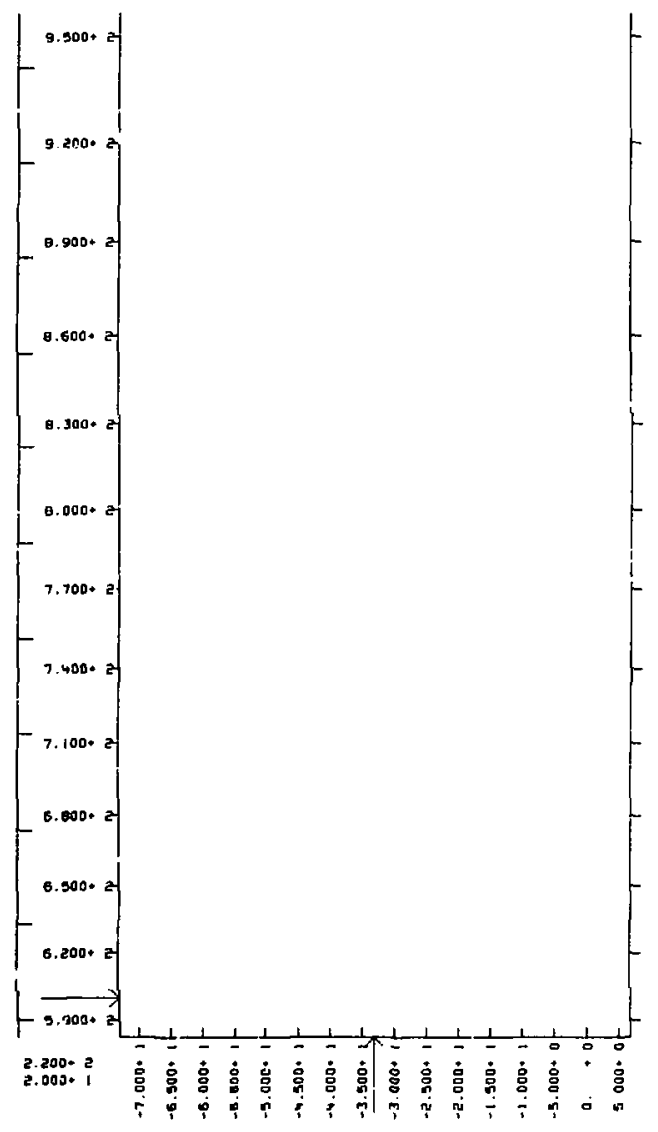




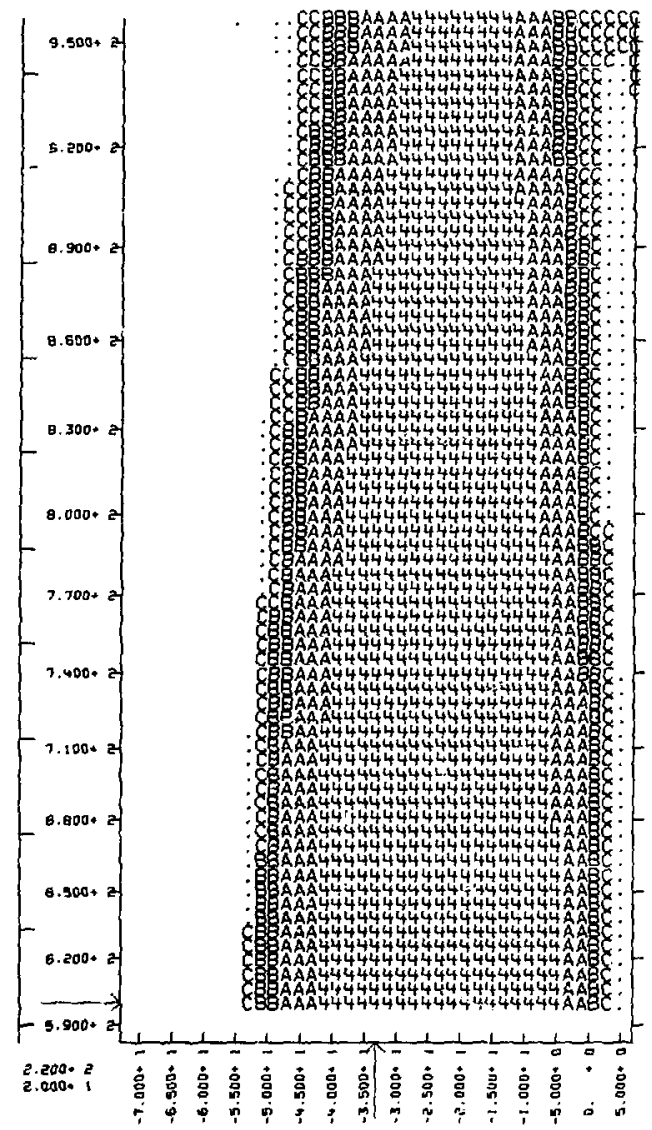




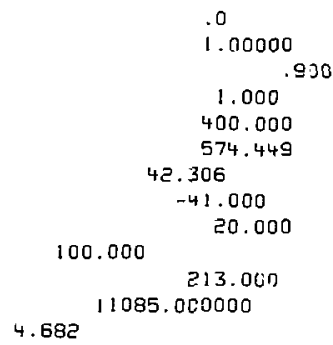

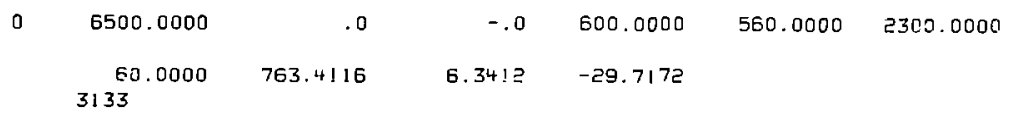

$-60-$ 


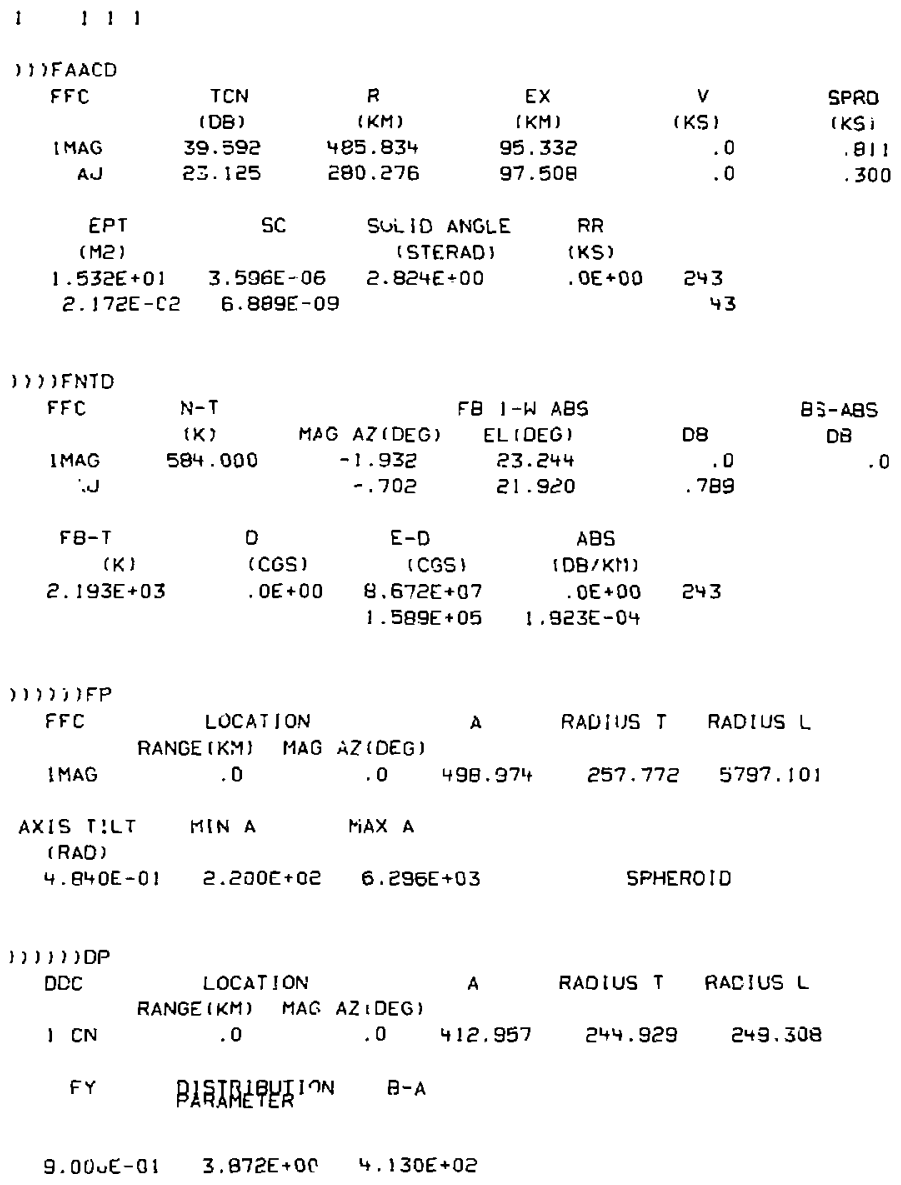




\begin{tabular}{|c|c|c|c|c|c|c|c|}
\hline 1 & 213 & 15 & 836 & 1.E5 & 21.9 & 5.3 & 19 \\
\hline 2 & -10 & 410 & 410 & 175 & 33 & 4.1 & 19 \\
\hline 3 & 1 & 60 & 22.5 & 295 & 325 & & \\
\hline 4 & 3 & 34 & 1 & 0 & 1 & 0 & 0 \\
\hline & 0000 & 00000000 & D & 60 & 8 & & \\
\hline 6 & 400 & -33 & 400 & D & 1 & .9 & ! \\
\hline 7 & 0 & 20 & 0 & 100 & 0 & 0001 & 0 \\
\hline 8 & -33 & 6500 & 0 & & 600 & 560 & 2300 \\
\hline $\begin{array}{l}9 \\
9\end{array}$ & $\begin{array}{r}1 \\
20\end{array}$ & $\begin{array}{l}1 \\
5\end{array}$ & 2 & 3 & 20 & 20 & 70 \\
\hline 0 & & & & & & & \\
\hline
\end{tabular}




$\begin{array}{rl}10.0000000000 & 7.0000000000 \\ 10.0000000000 & 7.0000000000 \\ 10.0000000000 & 7.0000000000 \\ 0 & 80 \\ -33.0000000000 & 1.0000000000 \\ 570.0000000000 & 0 . \\ 5500.0000000000 & 0 . \\ 2300.0000000000 & 1.5184364475 \\ 20.0000000000 & 5.0000000000 \\ 0 . & 0 . \\ 0 . & 0 .\end{array}$

20

0 .

1. 0000000000

0 .

D 


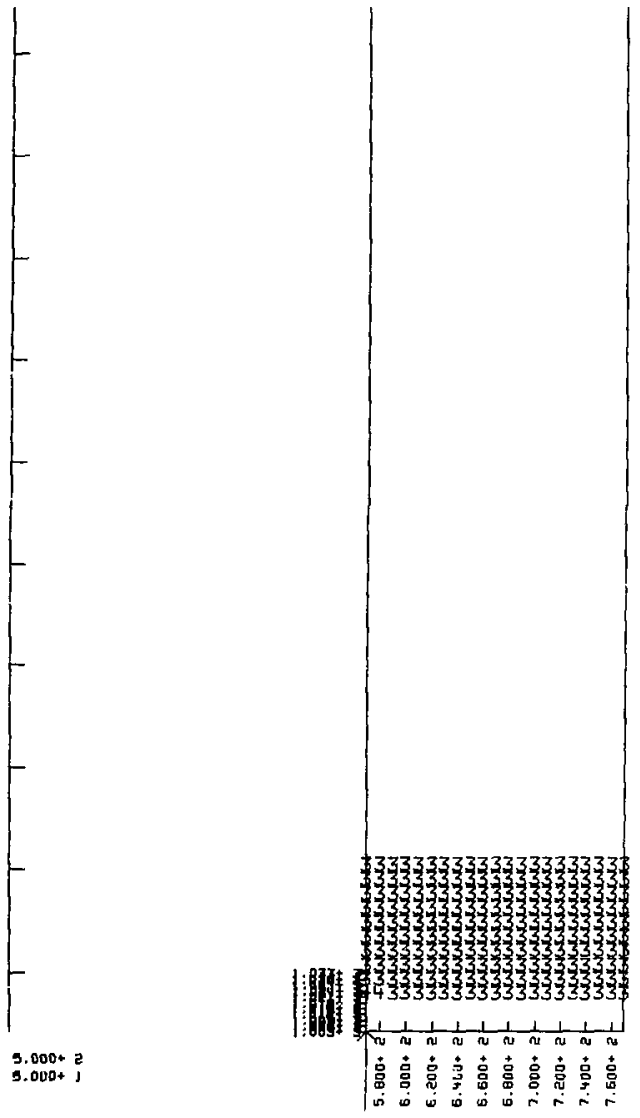




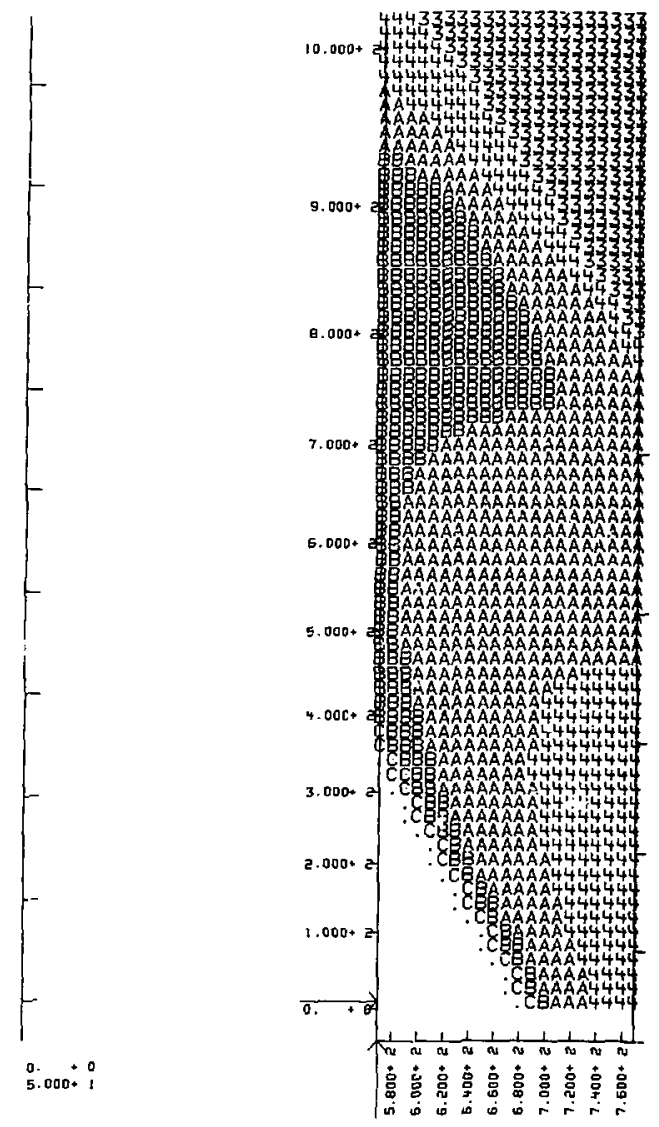




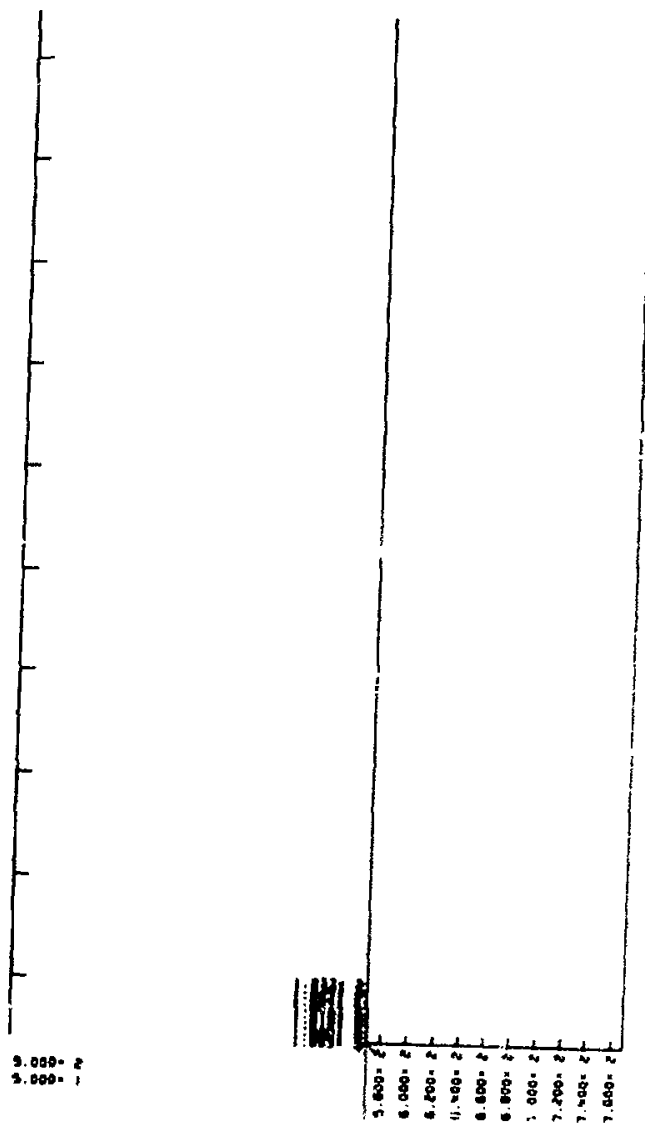

$-60-$ 


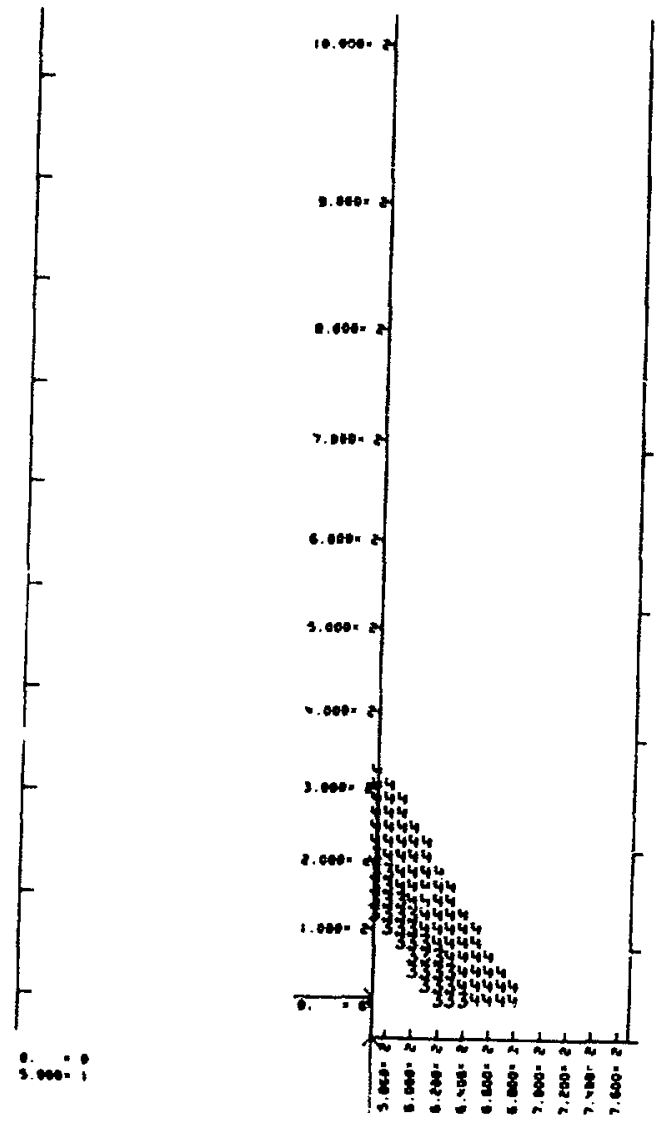




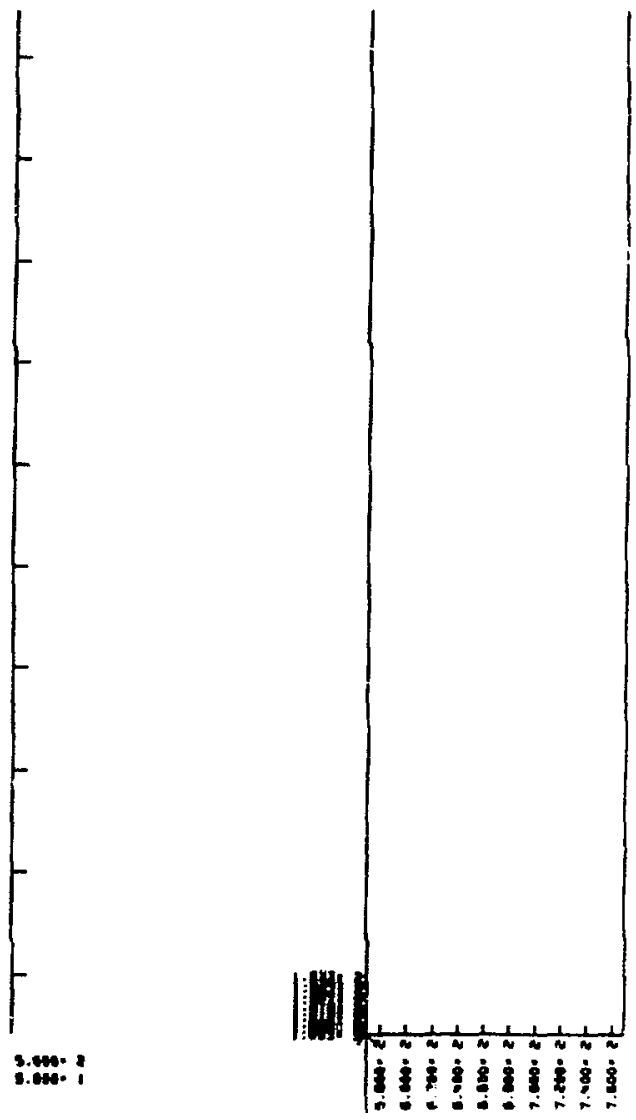




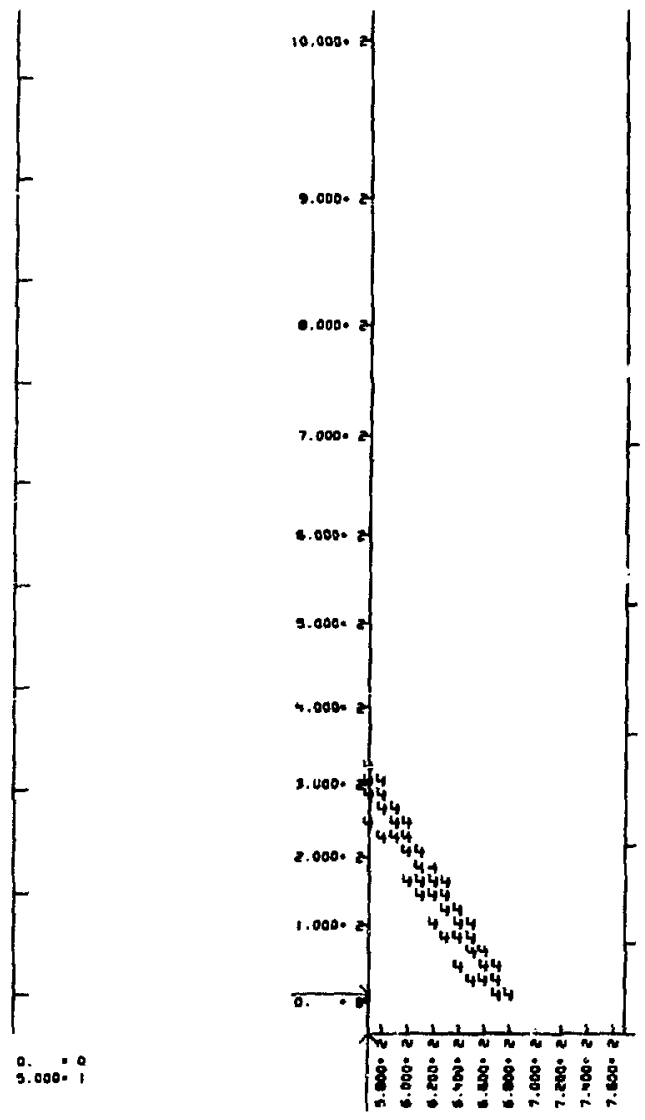





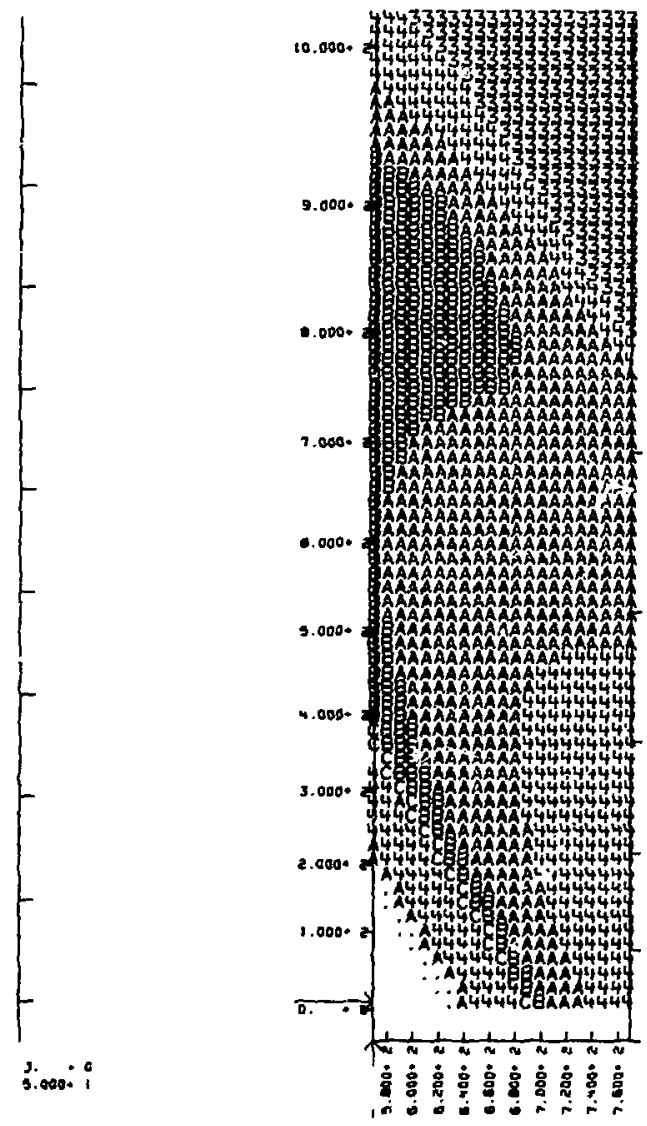




\section{$\ln \mid 1,1, \ldots$}

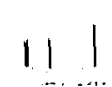

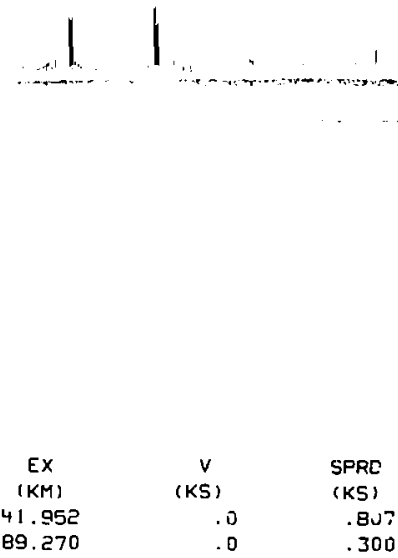

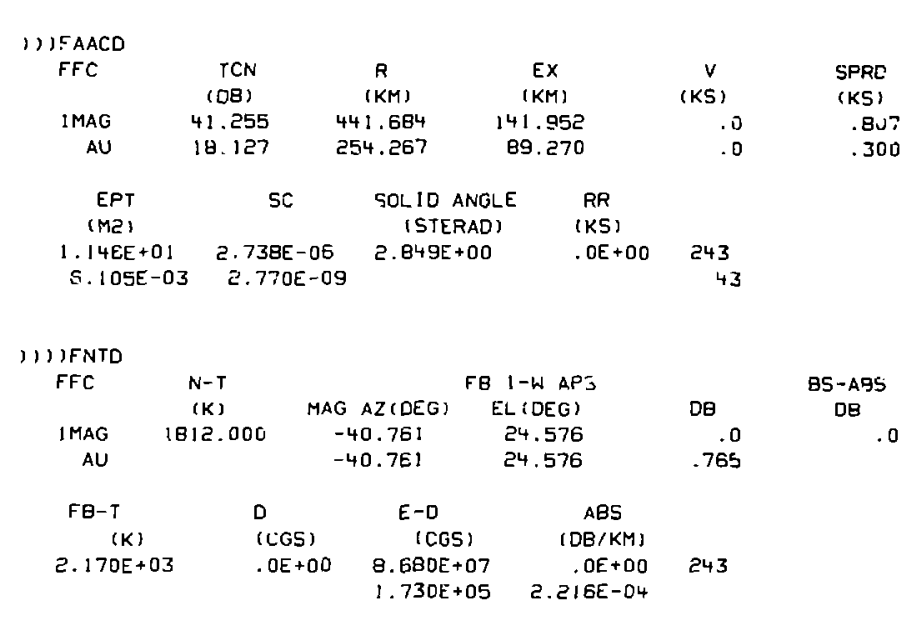

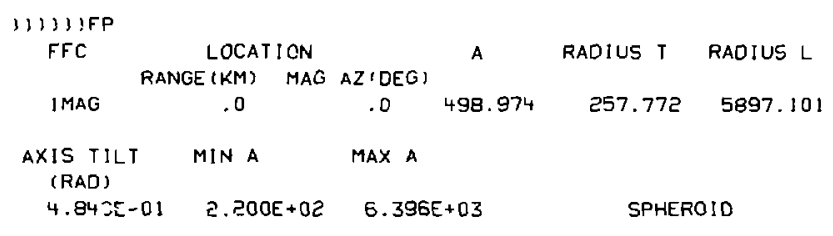

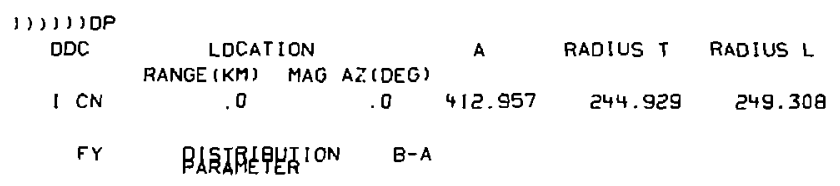

$9.000 E-01 \quad 3.855 E+00 \quad 4.130 E+02$ 


\begin{tabular}{|c|c|c|c|c|c|c|c|}
\hline 1 & 213 & 15 & 836 & 1.E5 & 21.9 & 5.3 & 19 \\
\hline e & -10 & 410 & 410 & 175 & 33 & 4.1 & 19 \\
\hline 3 & 1 & 60 & 22.5 & 295 & 325 & & \\
\hline 4 & 3 & 34 & 1 & 0 & 1 & 0 & 0 \\
\hline 5 & 0 & 0 & 0 & 60 & $B$ & & \\
\hline 6 & 400 & -33 & 400 & 0 & 1 & .9 & 1 \\
\hline 7 & 0 & 20 & 0 & 100 & 0 & 0001 & 0 \\
\hline 8 & -33 & 6500 & 0 & & 0000000 & 570 & 2300 \\
\hline 9 & 1 & 1 & 10 & 7 & 0 & 20 & 80 \\
\hline 9 & 20 & 5 & & & & & \\
\hline
\end{tabular}


EXAM PLE OF TY OVERLAY OPERATING ON A PALLOT INLINE MODE RUN

10.0000000000

10.0000000000 10.0000000000

0

$-33.0000000000$

570.0000000000

6500.0000000000

2300.0000000003

20.0000000000

0 .

0 .

$\begin{array}{ll}7.0000000000 & \\ 7.0000000000 & \\ 7.0000000000 & \\ 90 \quad 00 & 20 \\ 1.0000000000 & 0 . \\ 0 . & 1.0000000000 \\ 0 . & 0 . \\ 1.5194364475 & 0\end{array}$

5.0000000000

0 .
0 . 

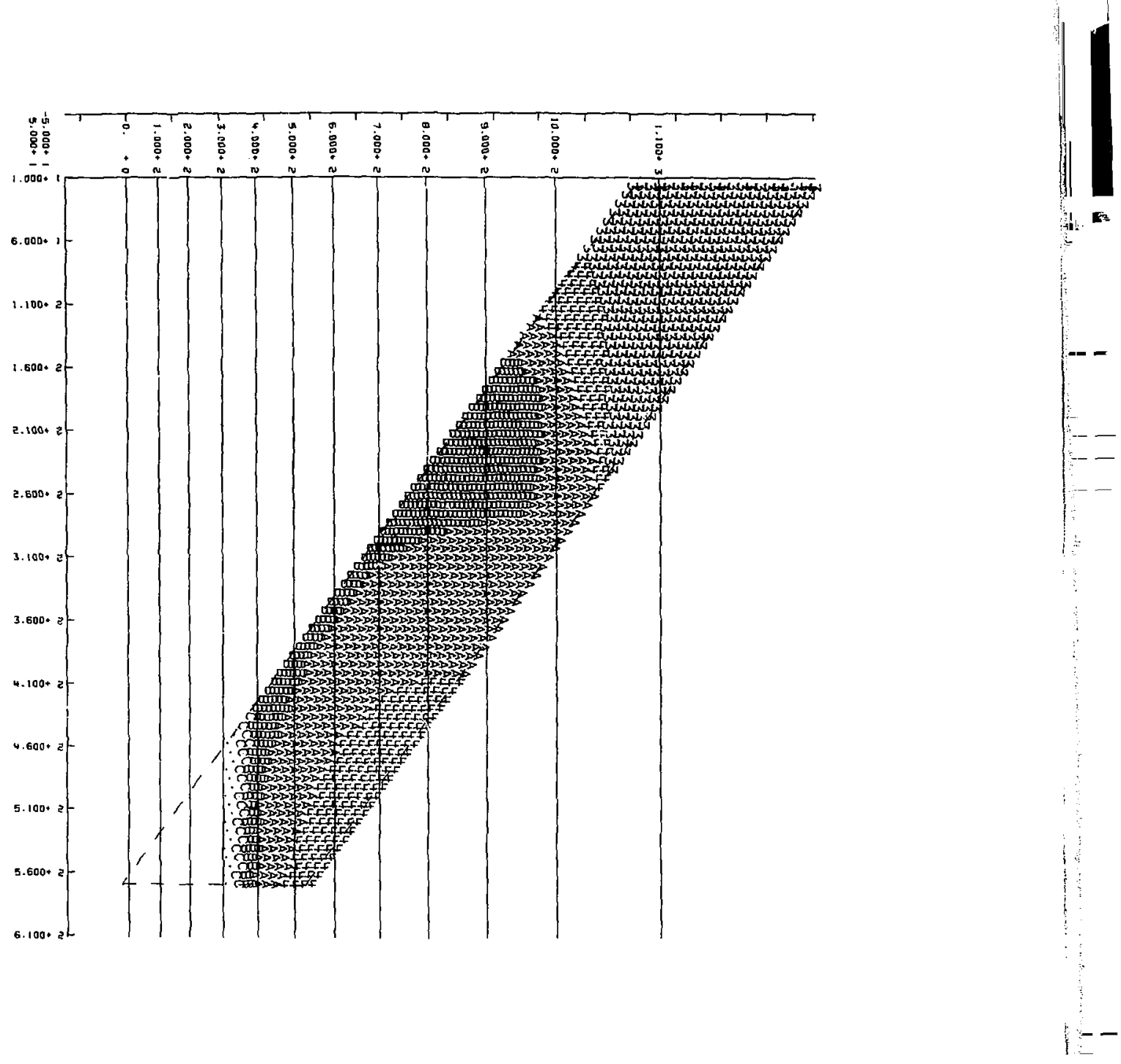


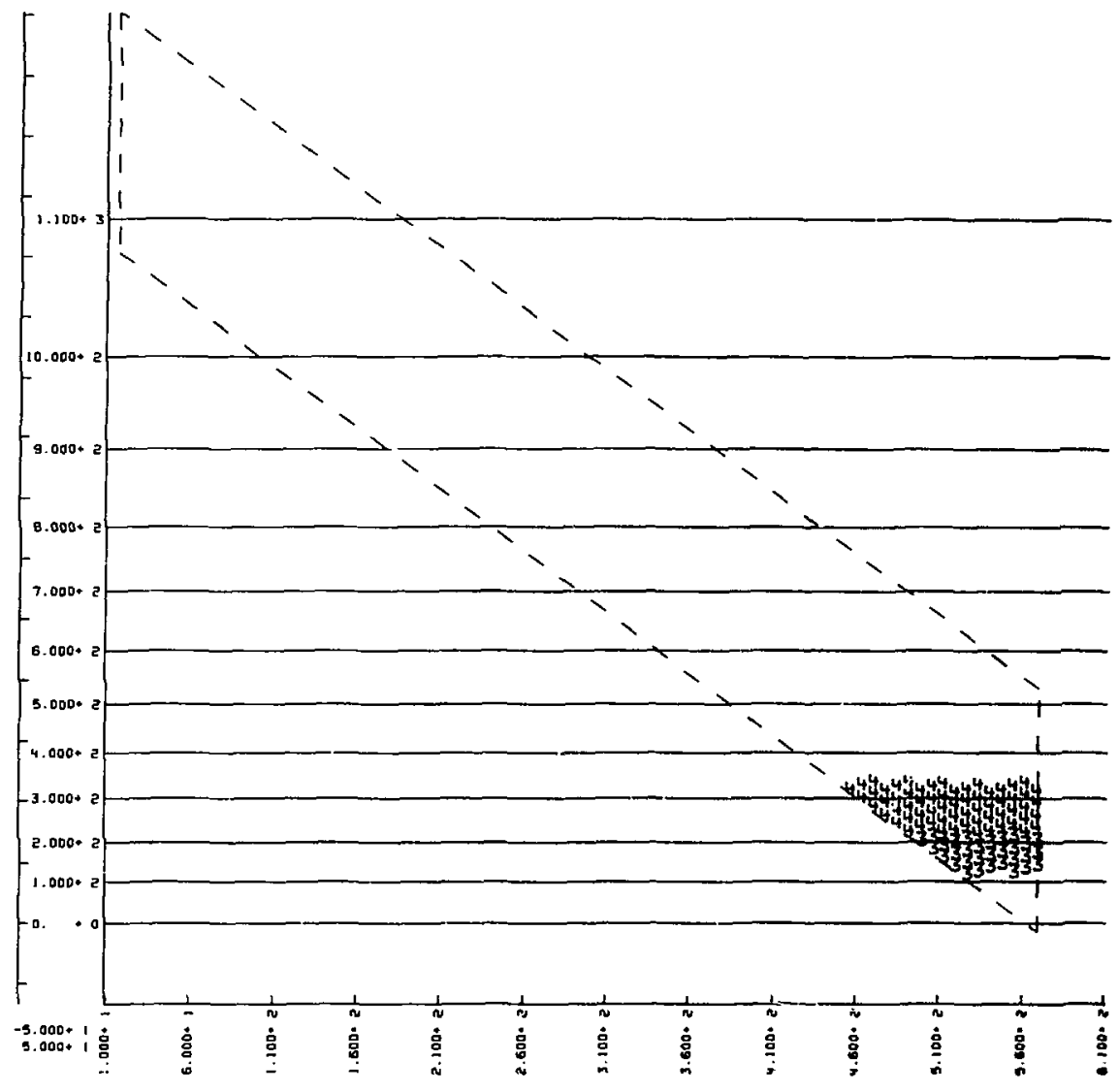




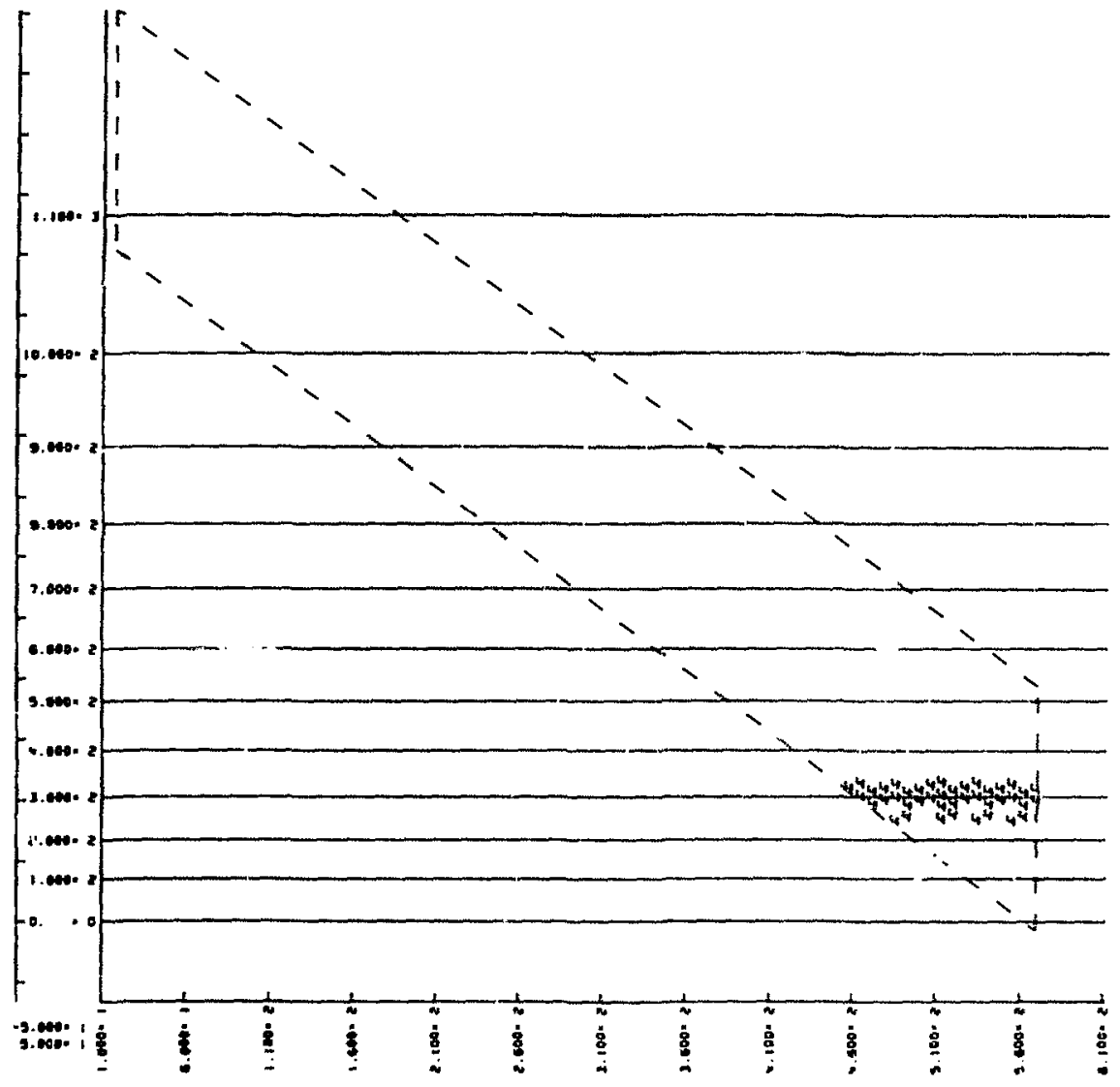




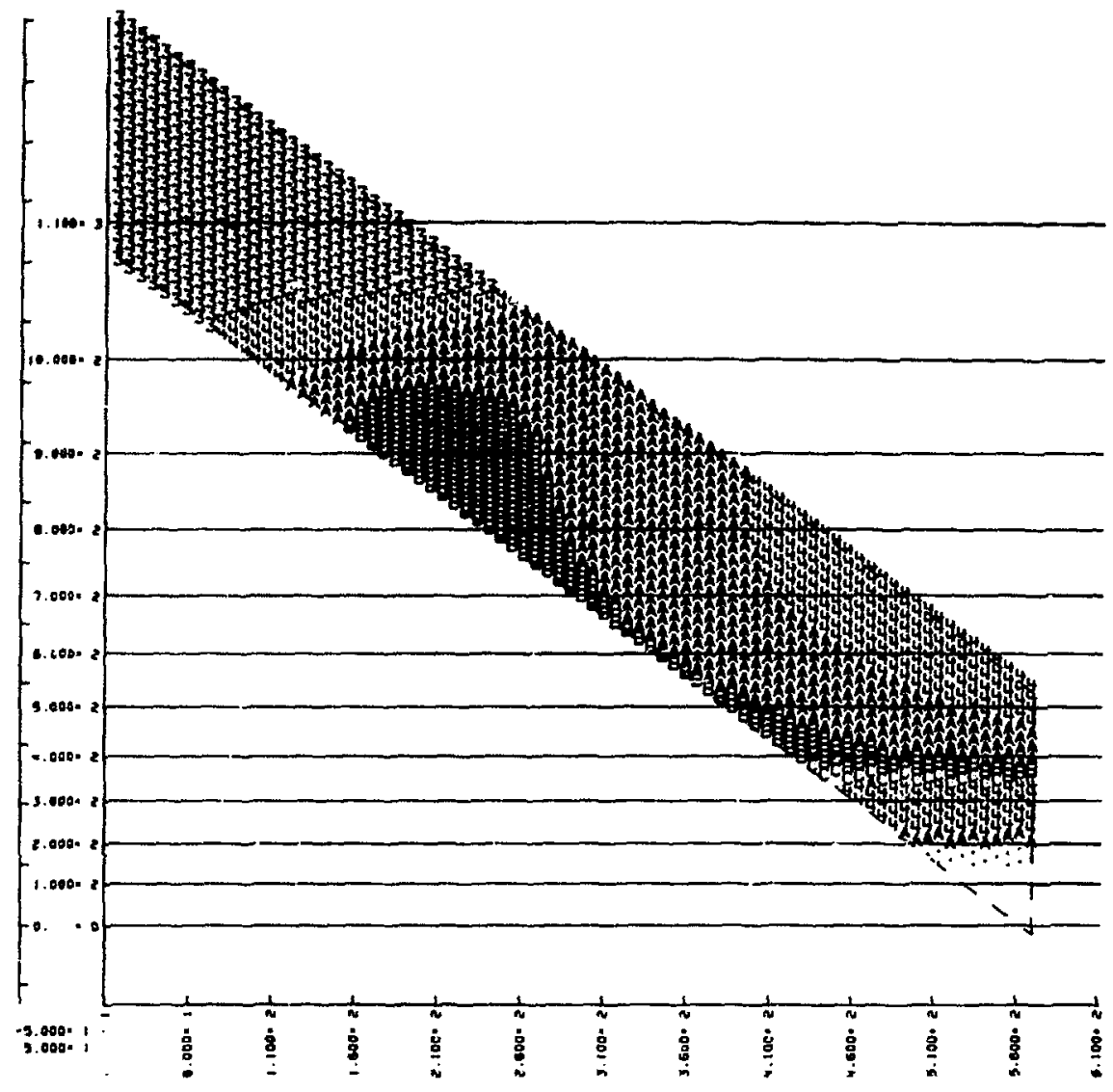




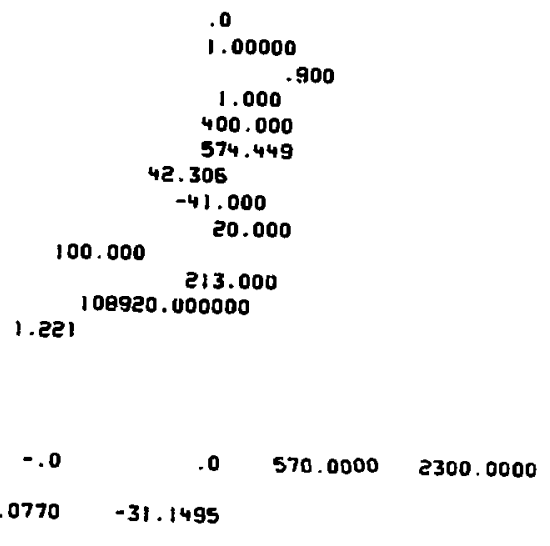




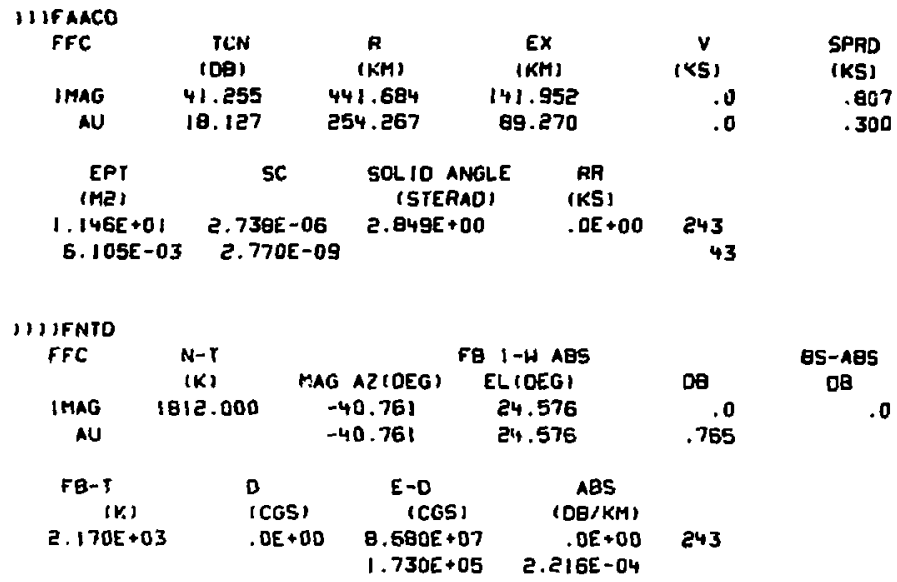

\begin{tabular}{|c|c|c|c|c|c|c|}
\hline \multirow[t]{2}{*}{ FFC } & \multicolumn{2}{|c|}{ LOCATION } & & $A$ & RADIUS $T$ & RADIUS L \\
\hline & IGE (KM) & MAG & $A 2\{O E G\}$ & & & \\
\hline IMAO & .0 & & .0 & 498.974 & 257.772 & 5897.10 \\
\hline $\begin{array}{l}\text { AXIS TILT } \\
\text { IAADI }\end{array}$ & MIN A & & $\operatorname{MAX} A$ & ] & OAL & \\
\hline
\end{tabular}

\begin{tabular}{|c|c|c|c|c|c|c|c|}
\hline \multirow{2}{*}{\multicolumn{2}{|c|}{ DOC }} & \multicolumn{2}{|c|}{ LOCATION } & & \multirow{2}{*}{$A$} & \multirow{2}{*}{ RAOIUS $T$} & \multirow{2}{*}{ RADIUS L } \\
\hline & & RANGE (KM) & MAG & AZ(OEG) & & & \\
\hline $\mathbf{I}$ & CN & .0 & & .0 & 412.957 & 244.929 & 249.308 \\
\hline & FY & \multicolumn{2}{|c|}{ 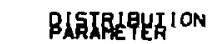 } & \multicolumn{2}{|l|}{ B-A } & & \\
\hline & 000 & \multicolumn{2}{|c|}{$3.855 E+00$} & \multicolumn{2}{|c|}{$4.130 E+02$} & & \\
\hline
\end{tabular}




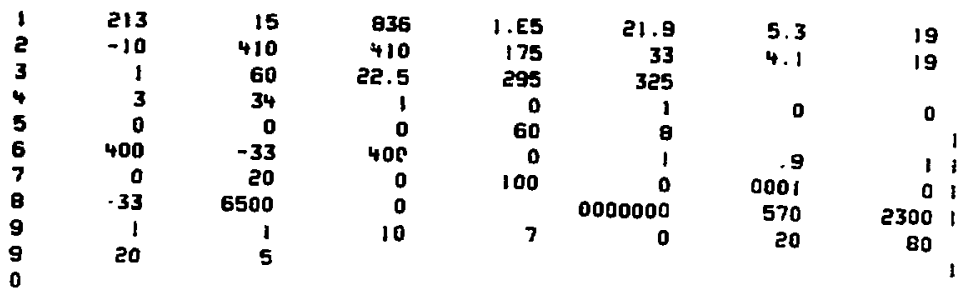


EXAM PLE OF THE OZ OVERLAY OPERATING ON THE COMPLETE TIME HISTORY MODE

5. 0000000000

5.0000000000

5.0000000000

$0 \quad 50$

$-33.0000000000$

0 .

6500.0000000000

2300.0000000000

20.0000000000

0.

o.

1
5. 0000000000

5. 0000000000

5,0000000000

$50 \quad 30$

1. 0000000000

0 .

0 .

1.5184364475

5.0000000000

0 .

o.
20

0 .

1. .0000000000

0 .

0 


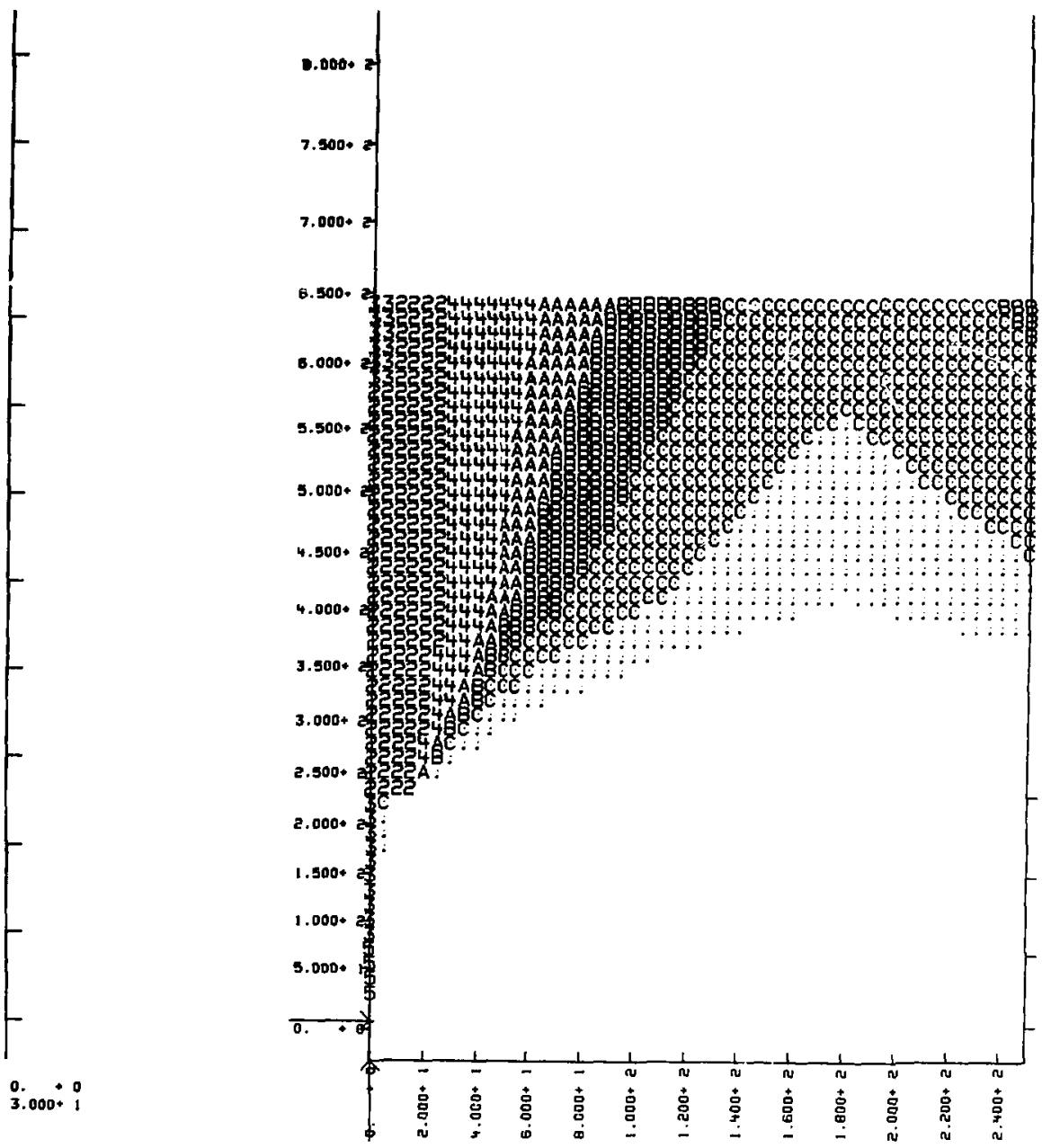




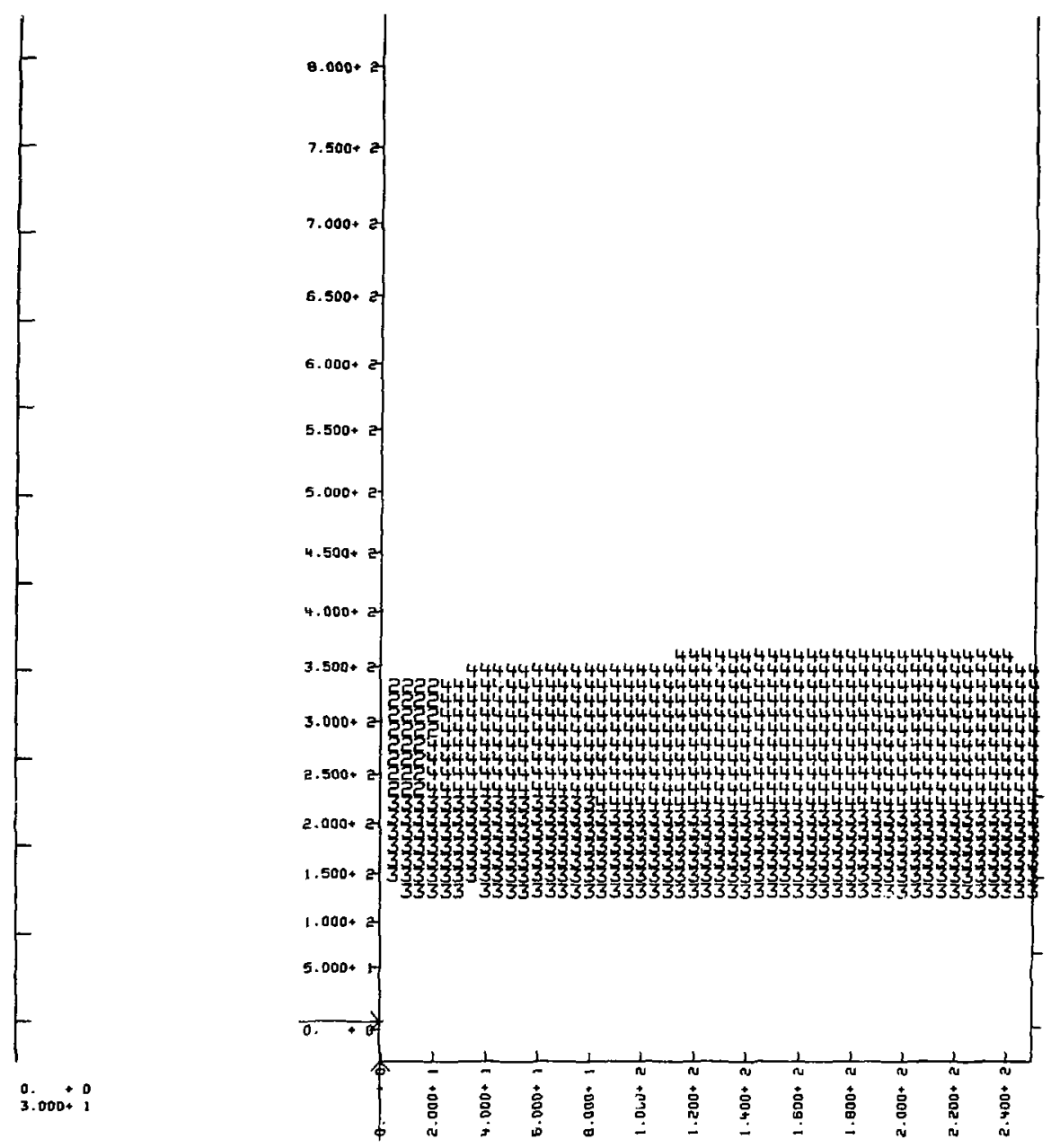




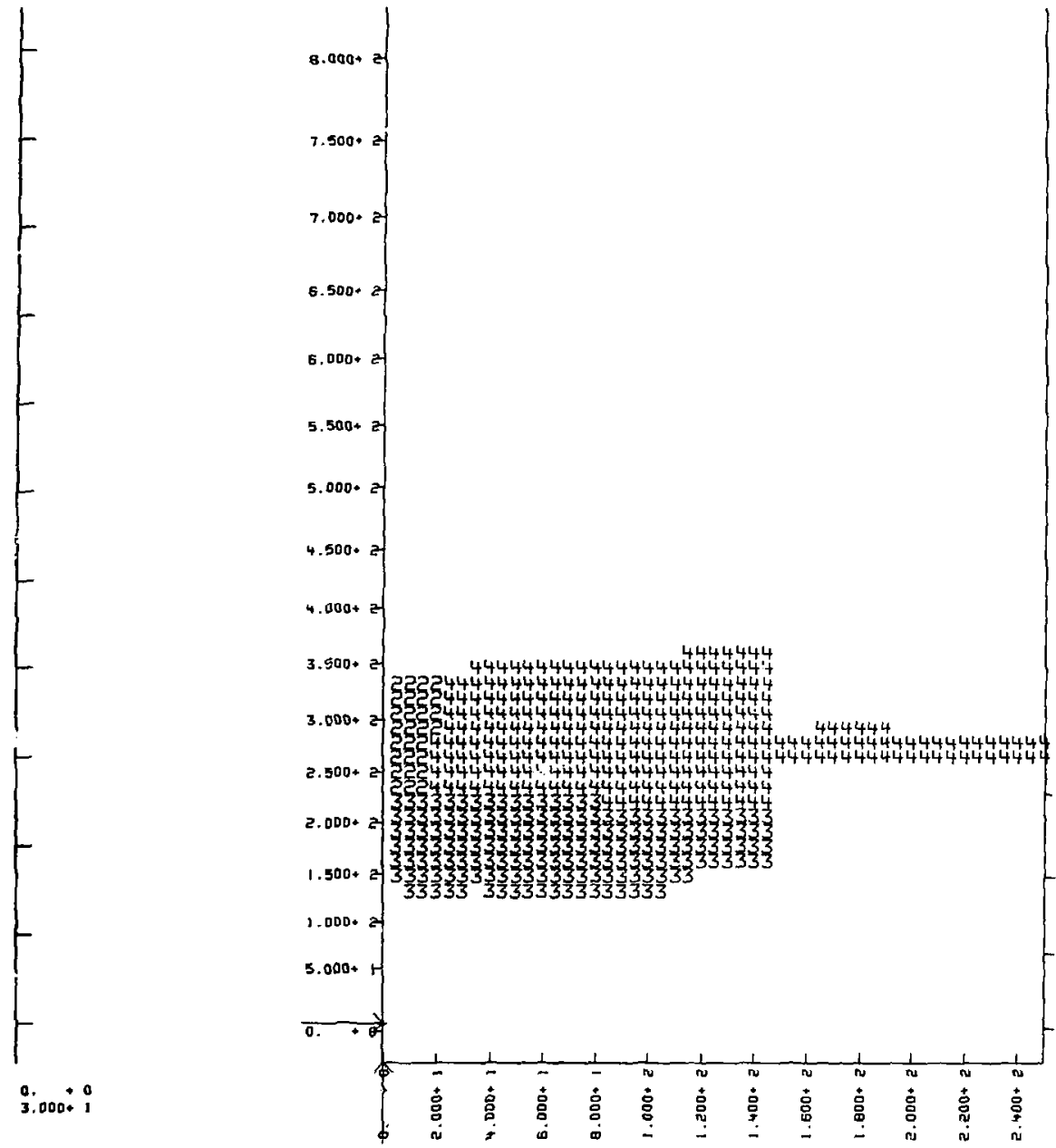




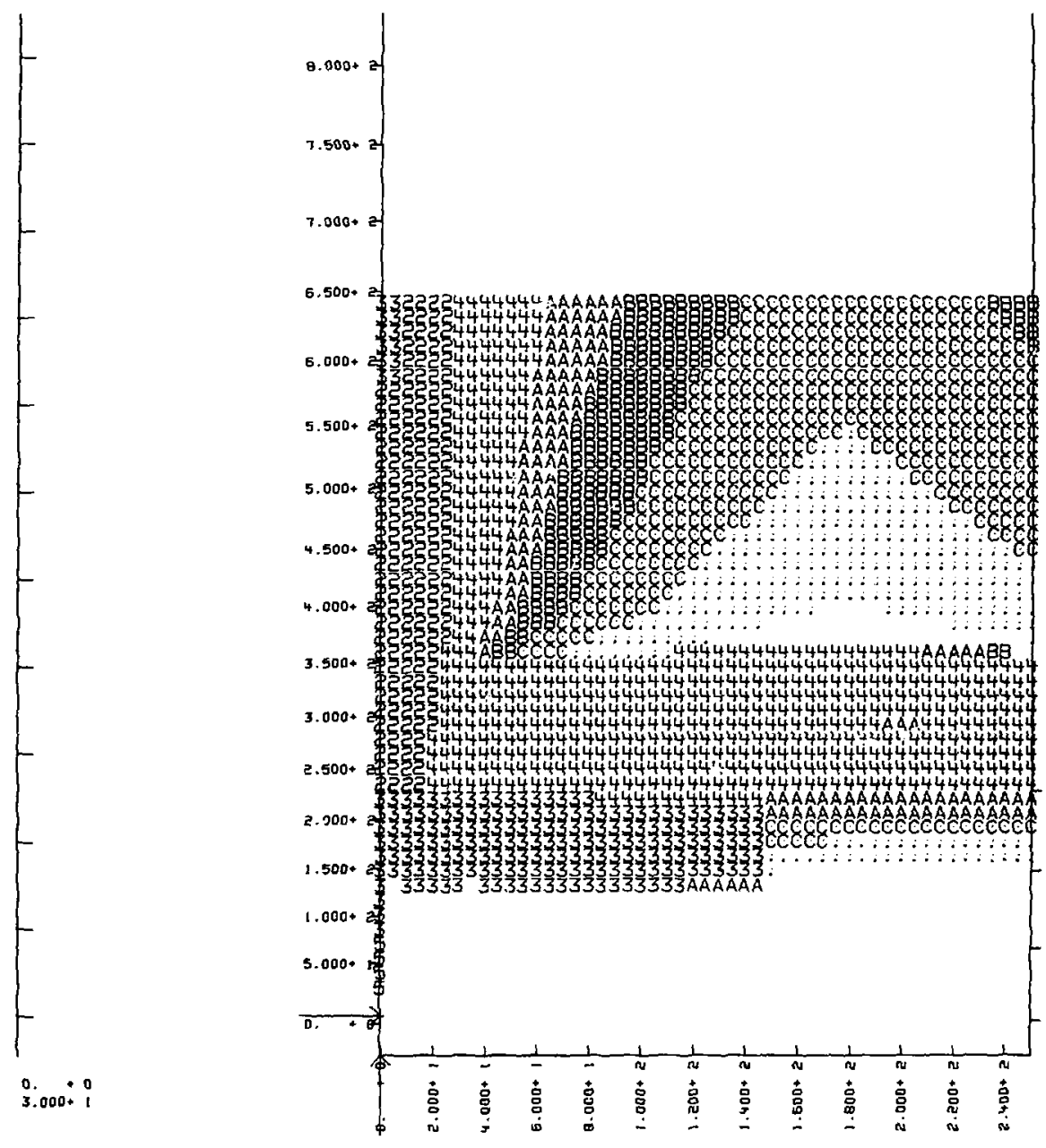




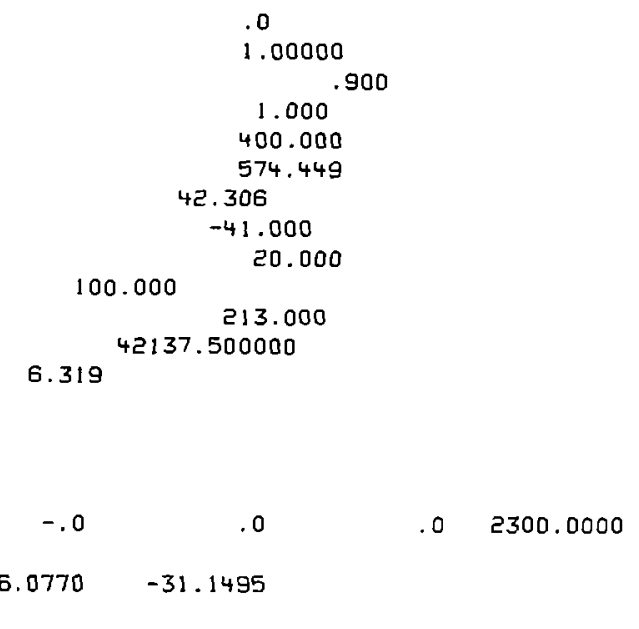


J J)FAACD

FFC

$$
\text { TCN }
$$

(DE)

$R$

EX

$\checkmark$

SPRO

I T I

73.342

405.134

(KM)

(KS)

(KS)

AU

203.953

51.913
72.707

.0

1. 865

\section{EPT}

(ME)

5C

SOL ID ANGLE (STERAD)

9. $667 E+06$

9. $912 E-01$

4. $493 E-07$

2. $548 E-13$

4. 727E-01

RR

(KS)

$. O E+00$

253

0

J) JRTO

FFC N-T

(K)

$$
\text { MAG AZ (DEG) }
$$

FB I-W ABS

EL (DEG)

31.846

$-41.000$

31.846

$D B$

I I
AU

$-41.000$

17.000

BS-ABS

DO

F日- T

(K)

1. $160 E+04$

$D$
(CGS)
$.0 E+00$

E-D

ABS

(CGS) (DB/KM)

3. $609 E+09$

1. $290 E+06$

2. $056 E+06 \quad$ 2. $550 E-03$

253

1) ) IFP

FFC

$$
\begin{aligned}
& \text { LOCATION } \\
& \text { RANGE (KM) MAG }
\end{aligned}
$$

$1 \mathrm{TI}$

.0

MAG

MIN A

$.0 E+00$

$.0 E+00$

SPHEROID

נगננगנग

DCC

LOCATION
RANGE (KM) IAG

FY BLRTREBUTION B-A

FY BLELRIBUIIION B-A

RADIUS T RADIUS L

A

400.000

242.995

242.995

$9.000 E-01 \quad 4.557 E+00 \quad 4.000 E+02$ 


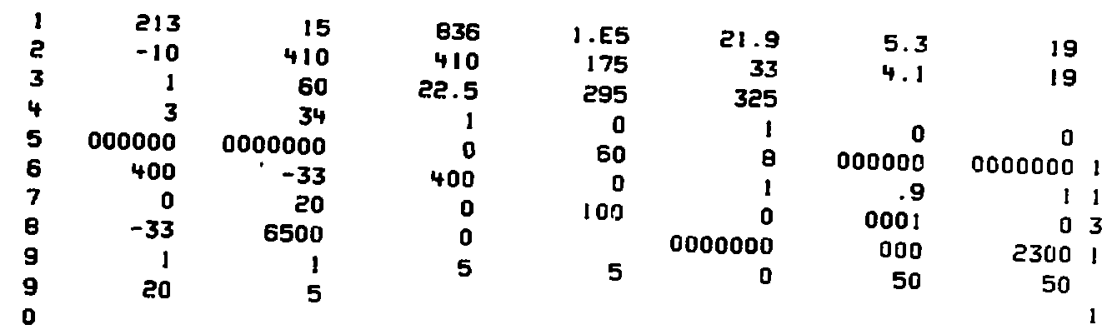




\title{
Appendix 4 \\ Lis tings of the OZ and TY Overlays
}

\author{
THE OZ OVERLAY LISTING
}

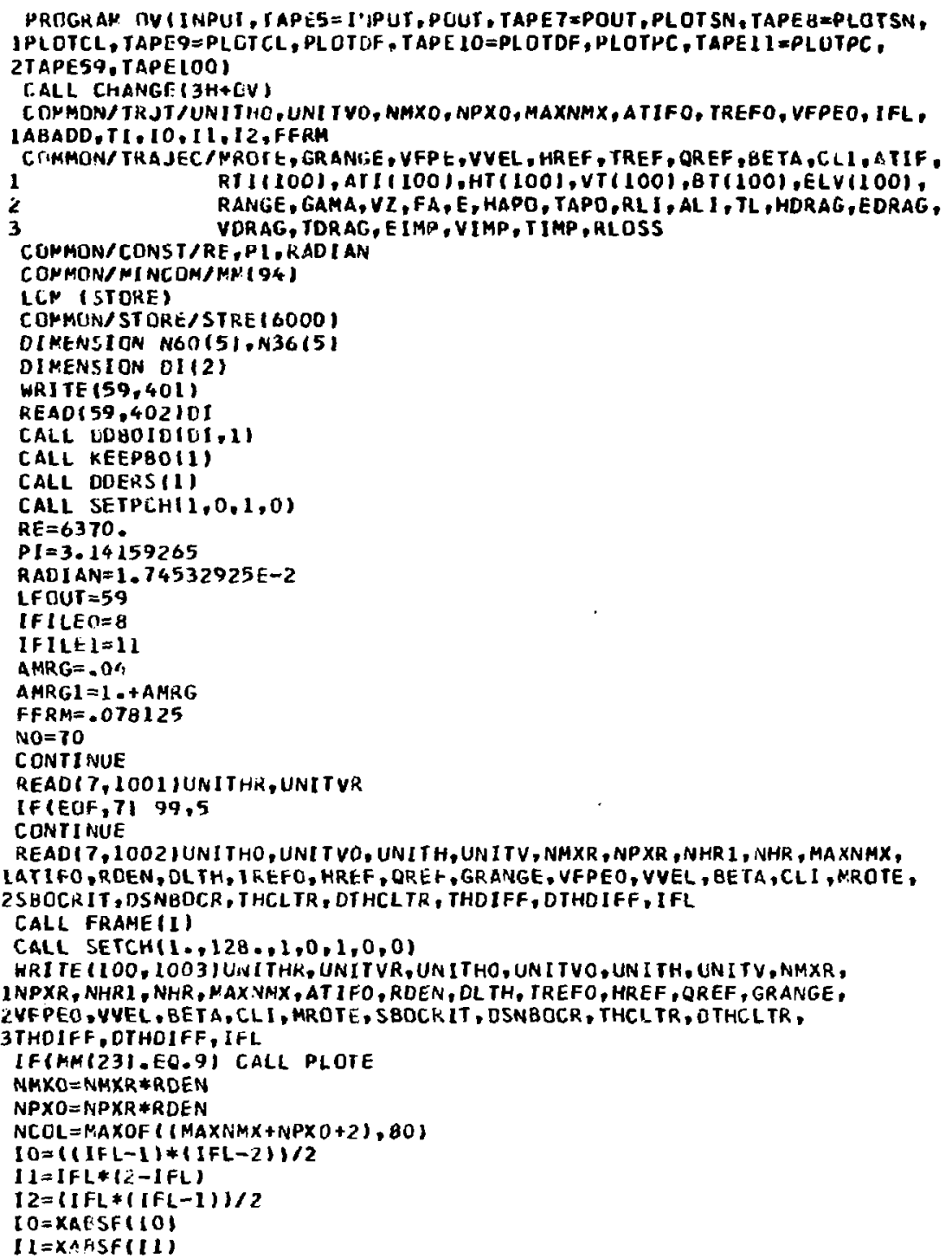


$12=x A B S F(12)$

$\triangle B A U D=A T I F O * I 0+T R E F 0 * 11+V F P E O * 12$

$N T=1$

ATIF = AT IFO-IO*NHXO*UNTTHO

TREF $=$ TREFO $-I 1$ HMXO $*$ UNI THO

VFPE $=$ VFPEO- I2*NHXO\#UNI THC

CALL TRAP INT, TREFO, LFOUT I

$I I=T I N P$

$A O=A B A D D-($ MAXNMX + I) + UN I THO

$A 2=A O+N C O L+U N I T H O$

$A 2 M=A$ ? + UNI THO

$A O M=A O$

TP=-INHRL *RDEN-1) *UNIT VO+TREFO+DL TH

$T P=T I-T P$

NS $=$ NHR $*$ ROEN

$N L=N S / N O$

$N R=N S-N 1 * N O$

$N I=M J N O F(N L \cdot 5)$

I GOTO=0

IFINR.GT.OI GO TO 21

IF (N). GT.O) IGOTO=2

Go TO 12

11 CONTINUE

I GOTO= ?

12 CONTINUE

IF(IGOTO.EO.O) GO TO I

DO 30 IF ILE=IFILED, IFILEI

GO TO $(10,20), 1$ GOTO

10 CONTINUE

$T 1=T P$

$T O=T I+($ NO $-N R I * U N I I V O$

$T 2=T 0-(N O-1)+$ UN I TVO

T2M=T2-AMRG* (TO-TZ)

CALL FRAME III

CALL MAPIAOM, A2H, T2H,TO,FFRM, L .FFRH, L I

IFIIFILE.GT.IFILEO) GO TO 31

CALL PLOTEA

CALL AXISIAOM,AZM, T2M,TO)

CALL SAYEQ(NGO(1), STRE (1),N36(1)

31 CONTI NUE

CALL PLOTEA

CALL DPLOTIN6OI),STKE (1),N36(1))

NRO $=$ NR

IF INI - EQ.OI NRD $=N R+1$

CALL UDPICT(IFILE, NRD, TI, AO)

IFINI.GT.OI GO TO 20

GD TO 30

20 CONTINUE

TO = TP $-N R * U N I T V O+N O * U N I T V O$

OD $2 L I=1, N I$

I PI $=1+1$

NSTRE $=I * 1200+1$

$T O=$ TO - NO $\#$ UNITVO

$T 2=T 0-(N O-1) * U N I T V O$

I $2 M=T 2-A H R G *(T 0-T 2)$

CALL FRAME I 1$)$

CALL MAPIAOH,A2M,T2M,TO,FFRM, I, FFRM, 1, )

IFIIFILE.GT.IFILEO\} GO TO 22

CALL PLOTEA

CALL AXIS(AOM, A2M,T2M,TO)

CALL SAYEDINGOIIPI), STRE(NSTRE),N36IIPI)

22 CONTINUE

CALL PLDTEA 
CALL (PL:JINGO(1H1), STRE(NSTRE),N361 IPI)

"IRU $=\mathrm{y}$

If II. W. VI) NRD=NL+1

:ALL I IPICTIIFIL, NKL, TO,AD)

2 I COATthat

SO COAJII,UE

I F I Lt $=7$

CALL IF LUE II)

CALL (DPICTUIIFIL:)

GO In

99 C.UNTINUE

CALl FRA:'E (1)

I ILE $=5$

CALL CIPICTUIIFIL:)

CALL PLCTE

CALL EXIT

401 FÜRMAT (2HIV)

40.2 FORMAI $(2.110)$

1001 FORMAI (/PF2O.1O)

1002 FURHAT $(2 F 20.10, / 2 F 20.10,75110.31 / 3 F 20.10), / 2 F 20.10 .110,31 / 2 F 20.10)$

$1 . /(10)$

1003 FURNII3(/2F20.10),/3I 10,3(/3F 20.10),/2F20.10,1 10,31/2F20.10),/110)

ENE

SUBRGUT INE TICK $(X 1, \times 2)$

CONMCIN/TCK/L(10O) , NTCK

SIGNS $=x 1 * \times 2$

$A=\times 2-\times 1$

$\Lambda L=A L C . G \perp O(A)$

$N=A L$

IF (AL -LT. O.) N=N-I

$l=A /(10 . * *(N)$

$\mathrm{NC}=\mathrm{C}$

IF (NC.G.T.5) NC=3

IF (NC.LT.I) NC=I

$T S=N C *(20) \neq(N-1)$

IFISIGNS.LI.O.I GU IU $T$

fi $\times 1=r$.

IF $|X|$.NE. $0.1 N X I=$ ALUULIO\{ABSF(Xl)

$E=X 1 /(10, * N \times 1)$

$N E=E$

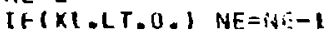

$\Sigma=N E$

$F=F *(10 . * N \times 1)-I S$

$I=0$

5 C.ONT I NUE

$E=\bar{T}+\mathrm{TS}$

IF(t.LT.XI) GO Tu 5

IF(E.GT.XZ) GO TIS 6

$I=\mathfrak{I}+\mathbf{L}$

$Z(I)=t$

(B) T(1) 5

6 C.UNTI NUE

NTCK $=1$

iEETUKN

7 CONIINUE

NTS $1=-x \perp /$ IS

กी $15 \mathrm{~J}=\mathrm{l}, \mathrm{NTS} 1$

$z(j)=-(\operatorname{sis} 1+1-J) * 12$

12 CONTI INUT

I $=$ NTS I

$E=-T S$ 
8 CONTINUE

$E=E+T S$

IF(E.GT.X2) GO TO 6

$I=I+1$

$2(1)=E$

GO TO 8

END

SUBRGUT INE DUPICT (NT APE,NO,TO, AO)

COMHON/TRJT/UNITHO, UNITVO, NMXO, NPXO, MAXNMX, ATIFO, TREFO, VFPEO, IFL, 1ABADO,TI, $10,11,12$, FFRM

I NTEGER AA

DIMENSION AAI851

$T=T O+U N I T V O$

DO $20 \mathrm{~J}=1$, NO

READ(NTAPE, 101 ) AA

101 FORMAT (85A1)

IF (AA (2).NE - IHN) GO TO 5

$J=N O$

GD TD 20

5 CONTINUE

$A=A O-U N I T H O$

$T=T$-UNI TVO

I TP=MINOF ( $($ MAXNMX +NPXO+2), 85)

DO $6 I=1 . I T P$

$A \approx A+U N \perp$ THO

CALL TRACECIAAYII,A,TI

6 CONTINUE

20 CONTINUE

RETURN

END

SUBROUTINE EFORM $(2 P, \angle N, N)$

INTEGER 2N

$N=0$

IF (ZP.NE,O.) N=ALOGIO(ABSF (ZP) )

$2 \mathrm{~N}=1 \mathrm{H}+$

IF (N.LT.OO) ZN=IH-

$Z P=2 P /(10 * * * N)$

$N=X A B S F(N)$

RETURN

END

SUBRDUT INE DDPICTOINTAPE)

INTEGER AA

DJMENSION AA(85)

$B=43$.

2 CONTINUE

READ (NTAPE, 1011 AA

IF (EOF, NTAPE) 99,1

1 CONTINUE

IF(AAI2I.EH.IHN) GO TO 99

IF(AA) (1).EQ.1HI) B=43.

$B=6-1$.

IF (B.GF.0.) GO TO 3

CALL FRAME (1)

$B=42$.

3 CONTINUE

CALL. SETCHI1, B, 1,0,1,0,0)

WR I TE (100, 101) AA

GO TO 2 
99 LIVT I NIE

QE TUR:

101 HORMAT(B5AL)

ENL

SUEKGUT INE HELP (SUBNAM, NST ATE, KOPT )

DIMENSION HOLRTH( 2$)$

DATA HCLRTH/3H , 3HNON/

WRI TE (59, 10$)$ HOLRI H(KEPT), SUBNAM,NSTATE

IFIKUPT.EO.1) CALL EXIT RETURN

10 FORMATIIHOA3,3THFATAL ERRDR DISCOVERED IN SUBROUTINE A6, 2OH AT STA I TEMENT NUMHER [6]

END

SUHROUIIVE TRAPIIVT, TSEC.LFOUTI

CDNMON/TRAJEC/MROTE, GRANGE, VFPE, VVEL, HREF, TREF, QREF, BETA, CLI, ATIF.

1

$\mathbf{2}$

3

COMMON/CUNST/RE, PI, RADIAN

RTI (100), ATI I100),HT(100), VT1100),BT(100),ELVI100),

RAVGE, GAMA, VZ, A, E, HAPO, TAPO, RL I , AL I, TL, HDRAG , EDRAG,

DIMENSION TSECIIOO)

RANGE $=$ GRANGE

$V Z=V V E L$

GAMA $=$ VFPE

$G=0.0098$

SPIN=7. 292E-5

IF (MROTE.NE.OI SPIN=0.

GAMMA $=$ GAMA*RADI AN

$\checkmark 2 M A X=2, * G * R E$

GRO2 $=G * R \div * R E$

I F I-R ANGE I $2,7,7$

$2 T H Q=R A N G C / R E * 0.5$

C THO $=$ CUS ( THO )

STHO= SIN(THO)

IF (GAFMA) $3,3,31$

3 IF $(V Z) 4,4,21$

4 IF (CTHO) $, 5,6$

5 WRITE(LFOUT.1005)

NHELP $=4$

60109

$6 \quad A=(1 .+5 T H O) * R E * 0.5$

$E=(1 .-S T H O) / C T H O$

CGAMM A $=$ SORT (A/RE)

$V Z=S C R T(V 2 M A X * S T H O /(1 .+S T H O))$

GO TO 29

7 IFIGANMA.GT.0.0.AND.VZ.GT.0.0) GO TO 11 NHELP=T

9 WRITE ILFOUT, 1001$)$

CALL HELP(GHTRAP ,NHELP, 1$)$

$11 \vee 2=V Z * 2$

TEST $=V 2 N A X-V Z$

NHELP $=11$

IFITEST $12,12,13$

12 WRI TE (LFOUI, 1002 )

GO TO 9

$13 A=$ GRO2/TEST

CGAMMA $=$ COS $(G A M M A)$

$E=S O R T(1--V 2 * C G A M M A * 2 /(A * G))$

$I H U=A \operatorname{COS}(1)+A *(E * E-1, \mid / R E) / E)$

KANGE $=2$ - $* R E * T H D$

GU TO 39 


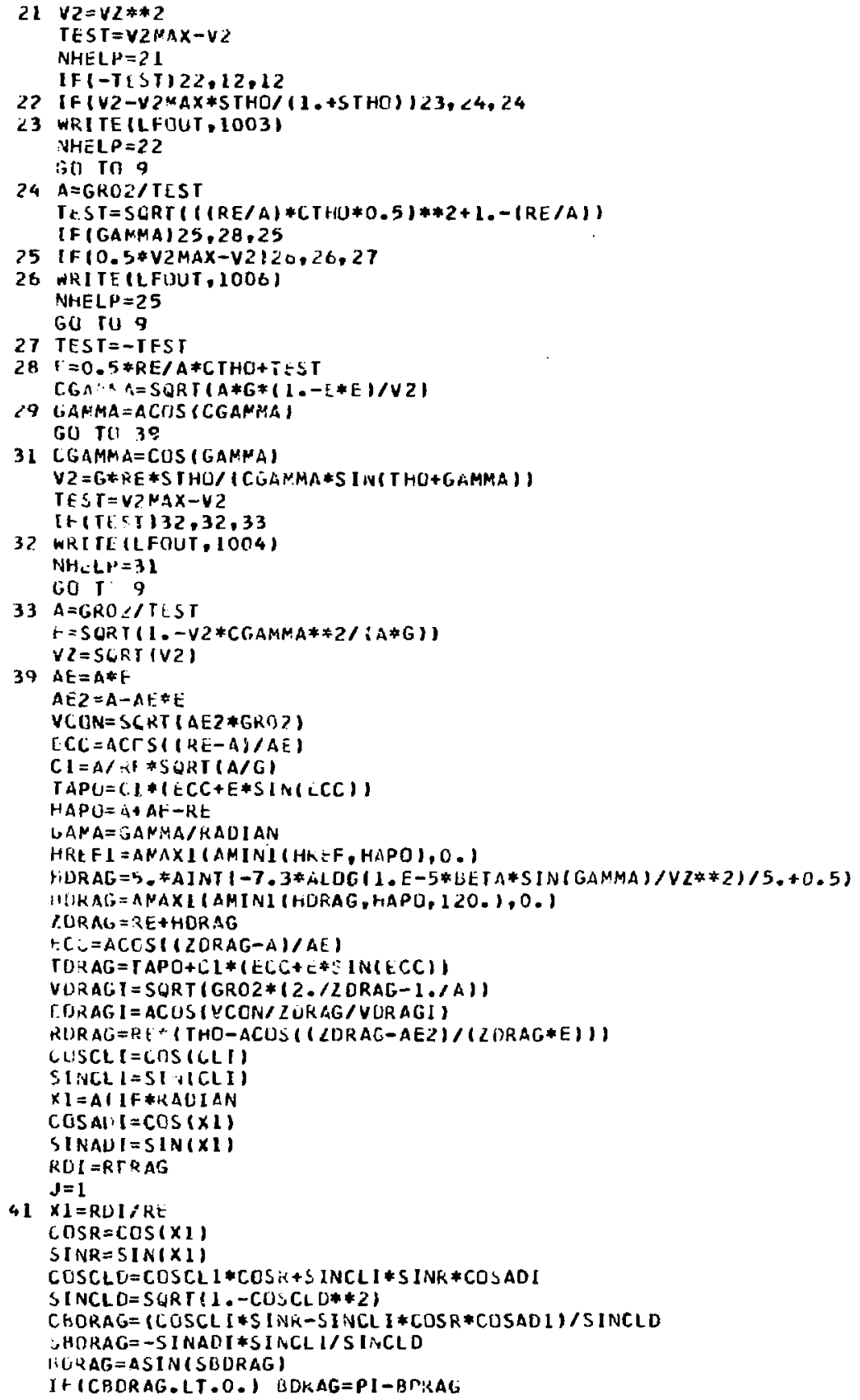


VATMIS $=S P I N \$ 2 D P A G \times S I N C L C$

$X I=Y L \times A G I * C U S(F C K A G I)$

HDRAG I = BIIRAG+ASIIU (VATMCS $C B D K A G / X I)$

VDRAG = SGRT I VDRAGI * $2+V A T M D S * 2-2$, \#VATMOS $\$$ XI*SINIBDRAGI)

FURAG=ASIN(VDRAGI*S INIEVRAGI)/VDRAG)

IF I HR EF 1-HORAG 151,52,52

51 IF IOREF $155,52,55$

$52 F C C=A C, S($ IRL+HREF $1-A) / A E)$

$T D E L=C l * 1 E C C+E * S[: V(E C C))$

IFIUREF.EO.0.) TUCL =-TDEL

TDEL $=T A P C T+T D E L$

$T I=T$ L KAG

$H I=H O R A G$

$V I=V[R A G$

$E I=E D R$. AG

$R I=R U I$

SO TO 56

55 TDE L $=1 . E 10$

CALL RVDRAGIG, BETA, TDRAG, HDRAG, VDRAG, EDRAG,RDI, TDEL,HREFI, VI, EI, IRI)

TI $=$ TUEL

$H I=H R E F I$

$56 \quad T L=T R E F-T D E L$

$T I=T E+T L$

$\operatorname{rIMP}=1 \cdot E 10$

$H F=0.0$

CALL RVDRAGIG, BETA, TI, HI, VI, EI, RI, TIMP, HF, VIMP, EIMP, RLOSSI

IF(O.0O1-ABS(RLOSS)) $57,58,58$

57 RDI $=R D I-R L G S S$

$\mathrm{J}=\mathrm{J}+1$

IF(J.GT.10) CALL HELP(6HTRAP, 57,1$)$

GO IO 41

58 TAPQ $=T A P O+T L$

$T$ TORAG $=\mathrm{T} D R A C+\mathrm{TL}$

RLOSS =RDRAG-RUI

CALL COORDX $10,1,1$, RDI, AT IF, HDRAG, X1, X2, X3, XDI, YDI, $201, \times 4, \times 51$

CALL ASPECTICOSCLI,SINCLI, 0, XOI, YOI, ZDI, $1,0,0 \ldots, \ldots \times I D, Y I D, Z I D I$

AID = ( BDRAGI - PI I/RADI AN

$T P=T L$

$I=0$

$601=1+1$

IF (NT) 60,95,60

$T I=T S E C(I)$

$61 E M=(I I-T A P Q) / C 1$

IF (TI-TL)62,62,63

$62 Z I=R E$

$\mathrm{HI}=0.0$

$V I=V Z$

$F I=G A N F A$

$R 1=R A N G E$

GO TU 68

63 IF(TI-TDRAG) $64,64,80$

$64 \mathrm{ECC}=\mathrm{EN}$.

$65 \mathrm{ECCl}=\mathrm{ECC}$

$F M I=E C C l+E * S I N(E C C I)$

$F C C=E C C 1+(E M-E M 1) /(1++E * \operatorname{COS}(E C C) 1)$

IF ( $\triangle B S$ ( ECC-ECCI)-1 E $E-6 * A A S$ ( ECC $) 166,66,65$

tot $2 I=A *(1+E * C O S(E C C))$

$H I=Z I-R E$

$V I=S G R T(G R 02 *(2 . / 2 I-1 . / A))$

$E I=A C C S(V C O N / Z 1 / V I)$

$R I=R E *(T H O-S I G N(A C O S((Z I-A E 2) /(Z[* E)), E M))$

$6 \&$ FTU $=R I-R Q R A G$ 
CALL COORDX(HDRAG, $1, K T C, A T D, H I, X 1, \times 2, \times 3, \times T D, Y T D, 2 T D, X 4, \times 5)$

CALL ASPECTICOSCLD, INCLD, HDRAG, XIC,YID, LID, I,XTD,YTO, ZTU,XTI, YTI, LIII

DLONG = SPIN*(TDRAG-AMAXI $I I, T L)$

$n L=A S I N(S I N C L I * S I N(0.5 * L L O N G))$

AI I =-ASIN(COS $\left.(0.5 * D L C N G) / C O S\left(x_{1}\right)\right) / R A D I A N$

IF (COSCLI.LT.0.) AII $=I 80 .-A I I$

$R I I=2 * R E * X I$

CALL COCRDX $(0, \ldots, R, R I I, A I I, 0, \ldots 1, \times 2, \times 3, X I I, Y I I, Z I I, \times 4, \times 5)$

CALL ASPECTICOSCLI, SINCLI, 0, XII, YII, ZII,I,XTI,YTI,ZTI,XTI, YTI,

12 TII

CALL COGRDX $(0,2, \times T I, Y T I, 2 T 1, R 1, A I, \times 1, \times 2, \times 3, \times 4, \times 5, \times 6)$

I F I IL-TII 71,71,87

$71 \operatorname{COSA}=-\operatorname{CUS}$ (BDRAGII

$X I=K r I / R E$

$\operatorname{COSR}=\operatorname{Cos}(\times 1)$

$S I N R=S I N(X I)$

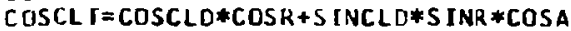

SI NLL. T = SURT ( $1,-$ COSCLT $* * 2)$

CUSY $=1$ COSCLD*SINR-S I NCL D*COSR*COSA I/SINCLT

$S$ I NB $=5$ I NCLD*SIN I BERAGI I/S INCLT

VATMOS $=S P I N * Z I \neq S I N C L T$

$X I=V(* \operatorname{COS}(E[)$

VII =SURIIVI**2+VATMOS**2-2**VATMOS*XI*SINB)

ETI=ASINIVI*SIN(EI)/VTI I

$\times 2=V T I * \operatorname{COS}(E T I)$

SINBT I $=(x) *$ S I NB-VATHOS $) / \times 2$

$\operatorname{COS} B T I=X_{1} * \operatorname{CoS} B / x_{2}$

Go To 9 I

BO IFITI-TIMP I8I,8L,B6

BL $H F=0,0$

I F I TP-I ORAG I $82,82,83$

B2 TP=TDRAG

HI =HORAG

$V T L=V D R A G$

ETI $=E T R$ AG

$R I=R D I$

83 CALL RVDRAGIG,BETA,TP,HI,VTI,ETI,RI,TI,HF, VTI, ETI,RI)

$\mathrm{HI}=\mathrm{HF}$

$T P=T I$

$A I=A T I F$

$X I=R I / R E$

$\operatorname{COSR}=\operatorname{COS}(\times 1)$

$S I N R=S I N(X 1)$

COSCL T $=$ COSCLI $*$ COS $H+S I N C L I * S I N R * \operatorname{COSAOI}$

SINCLT $=$ SORT I $1--C O S C L T * 2$ )

CDSBT I = (COSCLI*SINR-SINCLI $*$ COSR*COSADI $) / S I N C L T$

SI NBT I $=-5$ I NADI $\#$ S INCL I/ S INCLT

G0 TO 91

$86 R I=0.0$

$A I=0.0$

$H[=0.0$

Q 7 VT $I=0.0$

SI MBT $I=0.0$

COSBT I $=1.0$

ETI $=0.0$

91 IF $(1-N T) 92,92,96$

92 RII III =RI

AT $(1)=A I$

$H T(I)=H I$

$\checkmark T(I)=V T I$

HT (I)=ASINISINBTI)/RADIA,N

I F (COSBTI -LT . O.) HT (I) $=\$ 80 .-B T(I)$ 


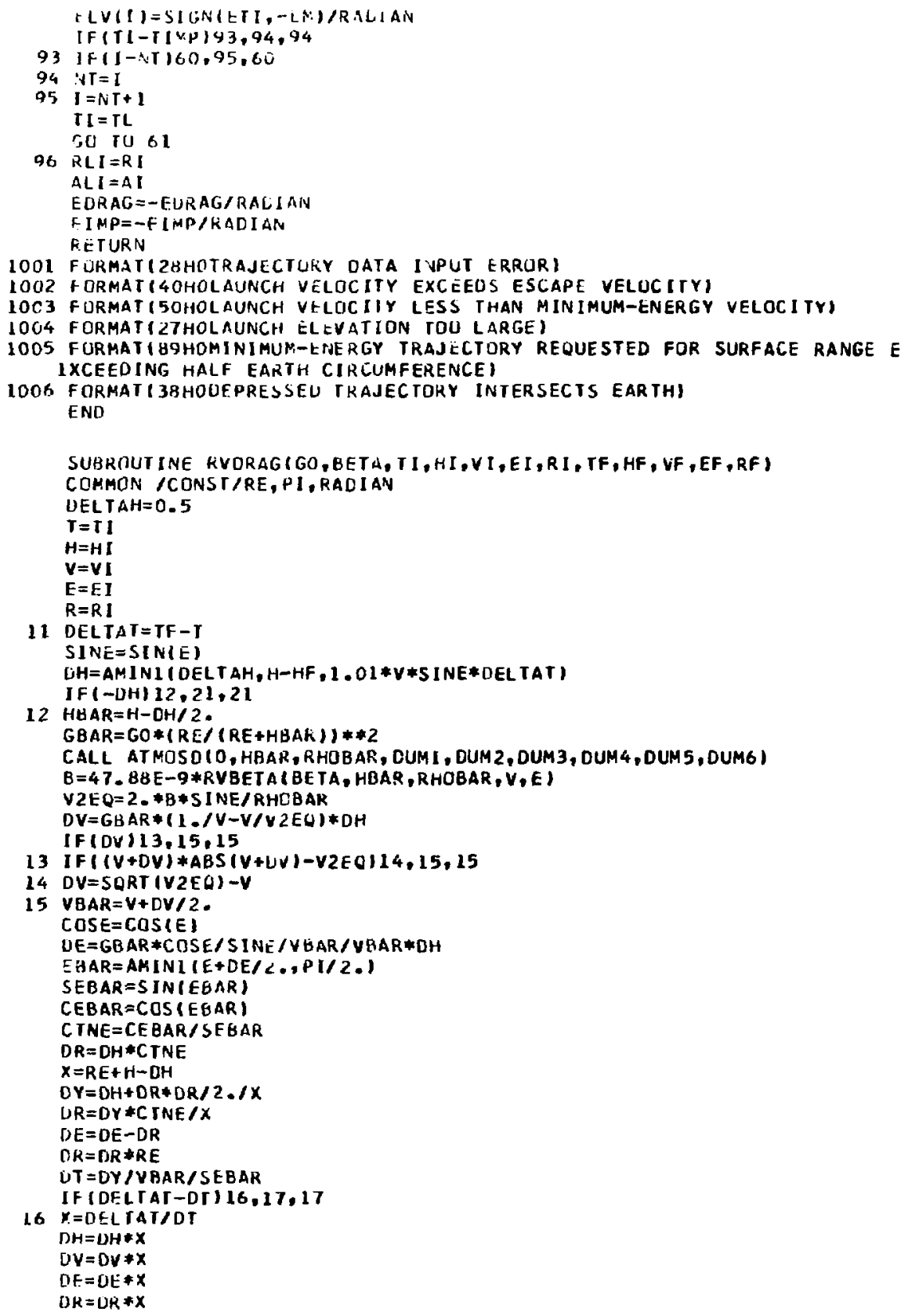


OT $=$ DELTAT

$17 \mathrm{I}=\mathrm{T}+\mathrm{O} \mathrm{T}$

$H=H-D H$

$V=V+D V$

$E=A M I N I(E+O E, P I / 2,1$

$R=R-U R$

$21 \mathrm{TF}=\mathrm{T}$

GR TO 11

$M F=H$

$V F=V$

$E F=E$

$R F=R$

RETURN

FND

SUBRDUTINE COORDX IHO,MOOE, RXSE, AYAA,HZEH, R, A, H, X, Y, Z, S,E) COMMON /CONST/RE,PI, RADIAN

REH=RE+HO

GO $\mathrm{TO}(11,21,31,41), \mathrm{MDOE}$

$11 \mathrm{R}=\mathrm{R} \times \mathrm{SE}$

$A=A$ Y AA

$H=H Z E H$

$U O=R / R E$

$U Z=A * R A D T A N$

$\mathrm{U} 3=\mathrm{RE}+\mathrm{H}$

$U l=U 3 * S I N(U 0)$

$X=U I * S I N(U 2)$

$Y=U 1 * \cos (U 2)$

$Z=U 3 * C U S(U O)-R E H$

IF (UO.EQ.0.) $Z=\mathrm{H}-\mathrm{HO}$

IFIUI $123,25,23$

2 I $X=R X S E$

$Y=A Y A A$

$Z=H Z E H$

$U 1=X * * 2+Y * * 2$

IF IUI) $22,24,22$

22. $U 2=R E H+2$

$U 3=50 R T(U 1+U 2 * * 2)$

$U I=S Q R T(U I)$

$R=A S I N(U 2 / U 3) * R E$

IF $(U 2 . L T=0.0) R=P$ I *RE-R

$A=A S I N(X / U I) / R A O I A N$

IFIY. 1T .0.0) $A=180 .-A$.

$\mathrm{H}=U 3$ 3-RE

$23 S=S U R T(U L * * 2+Z * * 2)$

$E=A S I N(Z / S) / R A C I A N$

GO TO 99

$24 R=0$.

$A=0$.

$\mathrm{H}=\mathrm{HO}+2$

$25 S=A B S(2)$

$\mathrm{t}=90$.

IF $(Z . L I .0 .0) \quad E=-90$.

GO 1099

$31 S=R X S E$

$A=A Y A A$

$E=H L E H$

$U 2=A * R A D I A N$

U3:E* I ADIAN

$U I=S * \cos (U 3)$

$x=U(* 5 I N(U 2)$

$Y=U 1 * \cos \left(U_{2}\right)$ 


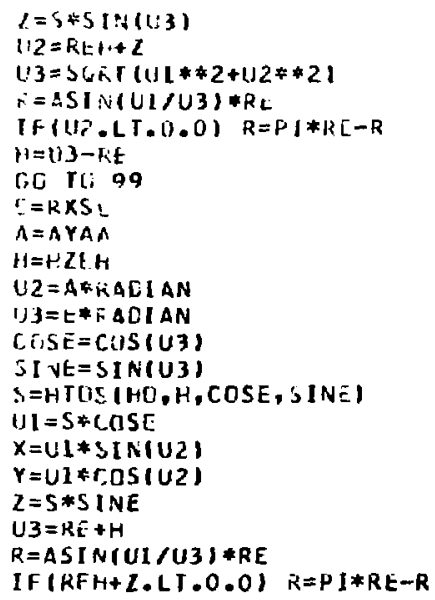


This subroutine is listed in Ref. 6.

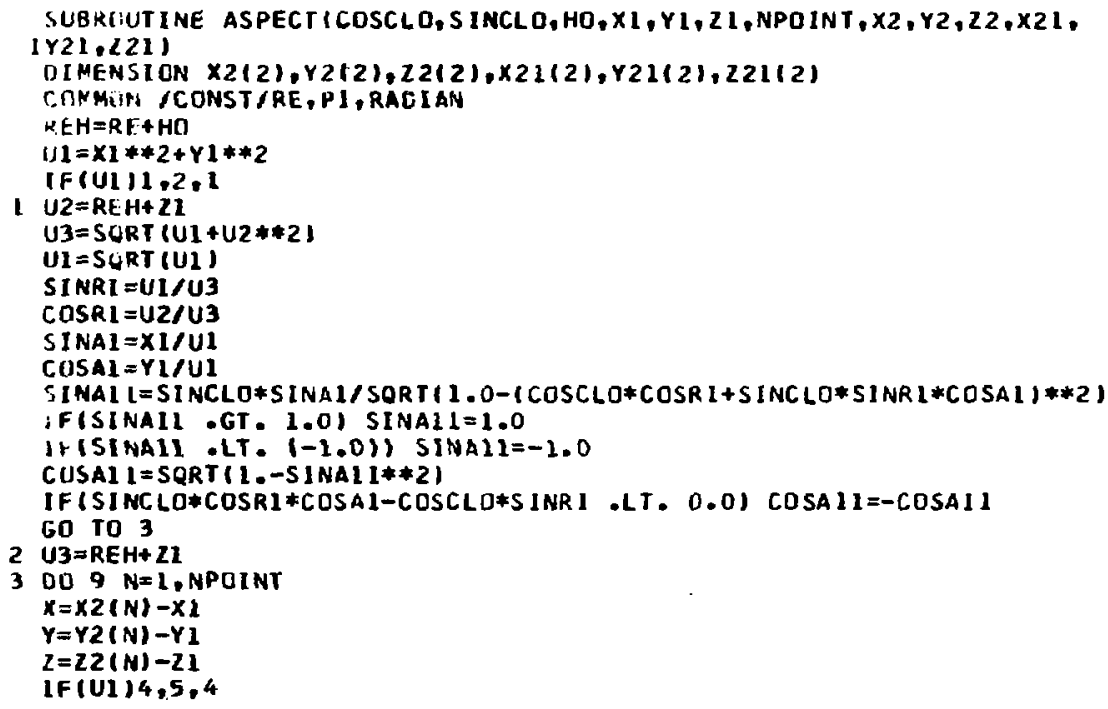




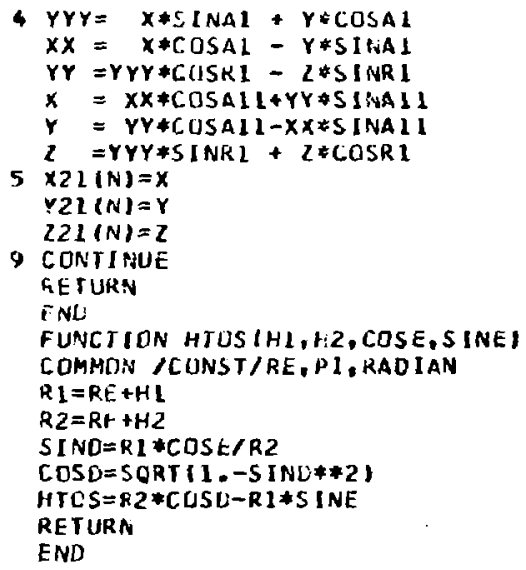

CALL LINEIXI,YI,XZ,YZ, 1,0) 
CALL IICKIYL,YZ)

$\times 2=\times 1+.015 *(B-A)$

C.U $20 \quad I=1$, NTCK

$Y 1=L(I)$

$Y 2=Z(I)$

20

CALL LINE $(X 1, Y 2, X 2, Y 2,1,0)$

$X=A-11.1 * F F R M *(B-A)) /(1,-F F R M)$

$Y=T 2-.025 *\left(T O-T_{2}\right)$

$Z P=2 \mid 1)$

CALL EFORM (2P, ZN,N)

CALL SETLCH $(X, Y, L, 0,0,0,0)$

WR I TE $1100,701 / 2 \mathrm{P}, Z N, N$

$Z P=2(2)-2(1)$

CALL EFORM $(Z P, Z N, N)$

WRI TE I 100,701 IZP, ZN,N

$D=(T O-T 2) / N T M I$

AT I F $=$ AT IFO - IO $\#$ NXO*UNI THO

TREF $=$ TREFO - I I*NAXO*UNI THO

VFPE $=$ VFP $E 0-I 2 * N M X O * U N I T H O$

OO $30 \quad I=1$, NT

TSEC $(1)=T I-T O+(1-1) * 0$

CALL TRAP (NTT, TSFCI [ I, LFUUT)

HTT (I ) = HT ( 1$)$

30 CONTINUE

DO $311=1, N T$

HT (1)=HTT (1)

31 CCNTINUE

$J O=1$

CALL TICK(HT(NT),HTII)

$X 1=A T I F *[0+T R E F * I 1+V F P E * 12$

$X=X 1-(.9 * F F R H *(\theta-A) \mid /(1 .-F F R M)$

$X 2=\times 1-.01 *(B-A)$

CALL LINE $(X I, T O, X 1, T 2,1,0)$

YKFFALT $=$ TI-TREF

IF ( Y YRFALT.GE.TZ) .AND, (YRFALT.LE.TO) ) CALL PLOTV(X,YRFALT,XI.

(YRFAL T)

LU $40 \quad \mathrm{I}=1$. NTCK

i) $50 \quad J=J 0, N T M I$

IF(ILII).GT.HT(J)).OR.IZ(I)-LT.HT (J+1) GD TO 50

$F=(H T(J)-Z(b)) /(H T(J)-H T(J+1))$

$Y=\operatorname{TI}-\operatorname{TSEC}(J)-F *(\operatorname{TSEC}(J+1)-\operatorname{TSEC}(J))$

$Y \mathfrak{l}=\mathbf{Y}$

$Y 2=Y 1$

CALL LINE $(X 1, Y 1, X 2, Y 2,1,0)$

$Z P=Z$ (I)

CALL EFORMIZP,ZN,N)

CALL SETLCH $(X, Y, 1,0,0,0,0)$

HRI TE (100,7OI IZP, ZN,N

$J=N T M L$

50 C.ONTI INUE

40 CONTI NUE

AT IF $=$ AT IFO+ IO*NPXO*UNI THO

TREF $=$ TREFO + I I *NPXO *UNI THO

VFPE $=V F P E O+12$ *NPXO *UNI THO

DO $61 \quad I=1, N T$

CALL. TRAP (MTT, TSEC (1), LFUUT)

HTTII) $=H T(L)$

61 CONTINUE

Lil $62 \quad I=1$, NT

HT (I) =HTT (I)

62 CONTINUE

$\mathrm{J} 0=1$ 
$X I=A T I F * I 0+T R E F * I I+V+P E * I 2$

$\times 2=\times 2+. C 1 *|8-4|$

CALL LINE $(X 1, T 0, \times 1, T 2,1,0)$

DC $60 \mathrm{I}=1, \mathrm{NTCK}$

i) $70 \mathrm{~J}=\mathrm{J0}, \mathrm{NTM}$

If(IL(I).GT.HT(J)). (UR. IZII).LT.HT(J+1)) GO 7070

$F=(H T(J)-Z(I) /(H T(J)-H T(J+1))$

$Y=T I-\operatorname{TSEC}(J)-F *(T \operatorname{SEC}(J+1)-T \operatorname{SEC}(J))$

$Y \mathfrak{I}=Y$

$Y Z=Y I$

CALL LINF $(X 1, Y 1, \times 2, Y 2,1,0)$

$J=N T M 1$

70 CONTINUE

GO CONTI NUE

RE TUR:

TU1 FURMAT (F6.3, A1, I2)

END 
PRDGRAM OVIINPUT, TAPES = INPUT, POUT, TAPE $7=P O U T, P L O T S N, T A P E B=P L O T S N$, 2PLOTCL, TAPEQ=PLOTCL, PLOTDF, TAPE 10=PLOTDF , PLOTPC , TAPE I $1=P L O T P C$, 2TAPES9, TAPEJ 001

CALL. CHANGEI3H+EV;

COMMUN/TRJT/UNITHO, UNITVO, NMXO, NPXO, MAXNMX, ATIFO, TREFO, VFPEO, IT I PPO , FFRM

COMMON/TRAJEC/MRUTE, GRANGE, VFPE, VVEL, HREF, TREF, OREF, BETA, CLI, ATIF, 1

2

3 RT I (100), AT L ( 100), HT $(100), V T(100), 8 T(100), E L V(100)$, RANGE, GAMA, VZ, A, E, HAPO, TAPD, RL I, ALI , TL , HDRAG , EDRAG ,

COMMON/CONST/RE,PI,RADIAN VDRAG, TDRAG, EIMP, VIMP, TIMP, RLOSS

COMMON/ MI NCOM/MM( 94$)$

LCM (STORE)

CDMMOM/STORE/STRE (6000)

DLMENSION N6O(5),N36(5)

DIMENSION TBII(100)

DIMENSION DI(2)

WRI TE $(59,401)$

READ (59,402)DI

CALL OOBOID(DI, I)

CALL KEEPBO (1)

CALL DDERS (1)

CALL SETPCH $(1,0,1,0)$

LFOUT $=59$

IFILEO $=8$

IF I LE I $=11$

$R E=6370$.

$P I=3.14159265$

RAOI AN $=1.74532925 \mathrm{E}-2$

AMRG $=.04$

AMRG1 $=1++$ AMRG

FFRM $=.078125$

USRST $=1024 . *(1 .-F+R M)$

$N O=85$

1 CONTINUE

READ ( 7, 1001 IUNITHR, UNITVR

IF (EUF, 7$) \quad 99,5$

5

CONTI NUE

READ ( T, 1002 IUNI THO, UNI TVO, UNI TH, UN I TV, NMXR, NPXR ,NHR I, NHR, MAXNMX, 1 ATI FO, RDEN, DLTH, TREFO, HREF, OREF, GRANGE, VFPEO, VVEL, BETA, CLI, MROTE, 2SBOCR IT, DSNBDCR, THCLTR, OTHCLTR, THO IFF, DTHO IFF, I FL

CALL FRAME (I)

CALL SETCHI $1,128, \ldots 1,0,1,0,0)$

WR I TE $(100,1003)$ UN I THR, UNITVR, UN ITHO, UNI TVO, UNITH, UNI IV, NMXR, INPXR, NHRL, NHR, MAXNMX, A T IFO, RDEN, DL TH, TREFO, HREF, QREF, GRANGE, 2VFPEO, VVEL, BETA, CLI, MROTE, SBUCRIT, DSNBOCR, THCL TR, DTHCLTR, 3THDIFF, UTHDIFF, IFL

IF(MM(23).EQ.9) CALL PLOTE NMXO $=$ NMXR *ROEN

$N P X O=N P X R * R D E N$

$T P=-($ NHR I *RDEN-1) *UNITVO+TREFO + DL TH

NS $=$ NHR $*$ RDEN

$N 1=N S / N O$

$N R=N S-N L * N O$

IF ( (NR+NI) -EQ.O) GO TO 1

$S L T=(N M X O+N P X O) \neq U N I T H O$

$H C=U S R S T /(1 N O+1) * U N I T V O)$

TANG = (USR ST*UNITVO* $($ NO+1) $/ 1$ AMRGL*IUSRST*SLT+(NO**2-1)*HC*

I (UNITYO)* $* 2)$ )

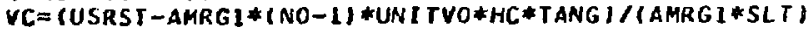


$N T=1$

AT IF $=$ AT IF O

TREF $=$ TREFO

YFPE $=$ YFPEO

CALL TRAP (NT,TREFO,LFOUT)

TI $M P O=T$ I $M P$

$N I P I=N I+I$

$0071 I=1$, NIPI

THI I (I) $=T$ I MPO- $(T P+N M X O$ *UNI THO+(I-1)*(NO+1)*UNITVO)

71 Continue

DD 72 IFI LE=I F ILEO, IFILEI

$0073 I=1$, W $1 P 1$

NSTRE $=(1-1) * 1200+1$

$T O=T P+(I-1) * N O * U N I T V O$

$T Z=T O+(N O-1)$ \#UNITVO

TOM $=$ T $0-U N I$ TVO

$T 2 M=T 2+U N I$ TVD

$T B I 0=T B I I(I)+S L T$

TQI $]=1 B(0-A M R G 1 *(S L T+((N O-1) * U N I T V O * H C * T A N G) / V C)$

CALL FRAMEII)

CALL MAPITOM,T2M,TBI 3,TEIO,FFRM, I, FFHR, I.)

IF (IFILE.GT. IFILEOI GO TO 74

CALL PLOTEA

CALL AXISITOM,T2M,TBI3,THIO)

CALL SAVEDINGOII), STKE(NSTREI, N36II)

74 CONTI NIUE

CALL PLOTEA

CALL DPLUT INGOIII, STRE (NSTREI, N36III)

$N R D=N O$

IF(I.EO.NIPI) NRD=NO+I

CALL DDPICTIIFILE, NKD, TO, TBIIII)

73 CONTINUE

72 CONTINUE

I FILE=7

CALL FHAME (1)

CALL DOPICTOIIFILE]

GU TU 1

99 CONTINUE

CALL FRAME (1)

IF I LE $=5$

CALL DDPICTOIIFILE)

CALL PLOTE

CALL EXIT

401 FORMAT(2HID)

402 FORMAT(2A10)

1001 FORMAT $(12 F 20.10)$

LOO2 FURMAT(2F20.10./2F20.10,15110,3(/3F20.10),/2F20.10,110,3(/2F20.10) $1,(110)$

1003 FDRMATI3(/2F20.10),/5I L $0.3(/ 3 F 20.10), / 2 F 20.10,110,3(/ 2 F 20.101, / 110)$ END

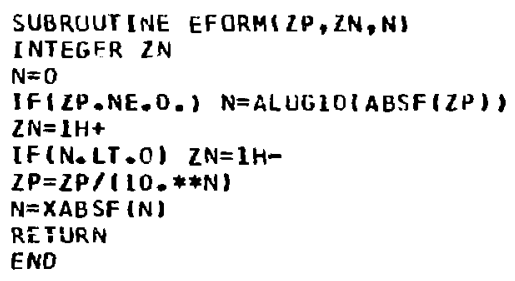




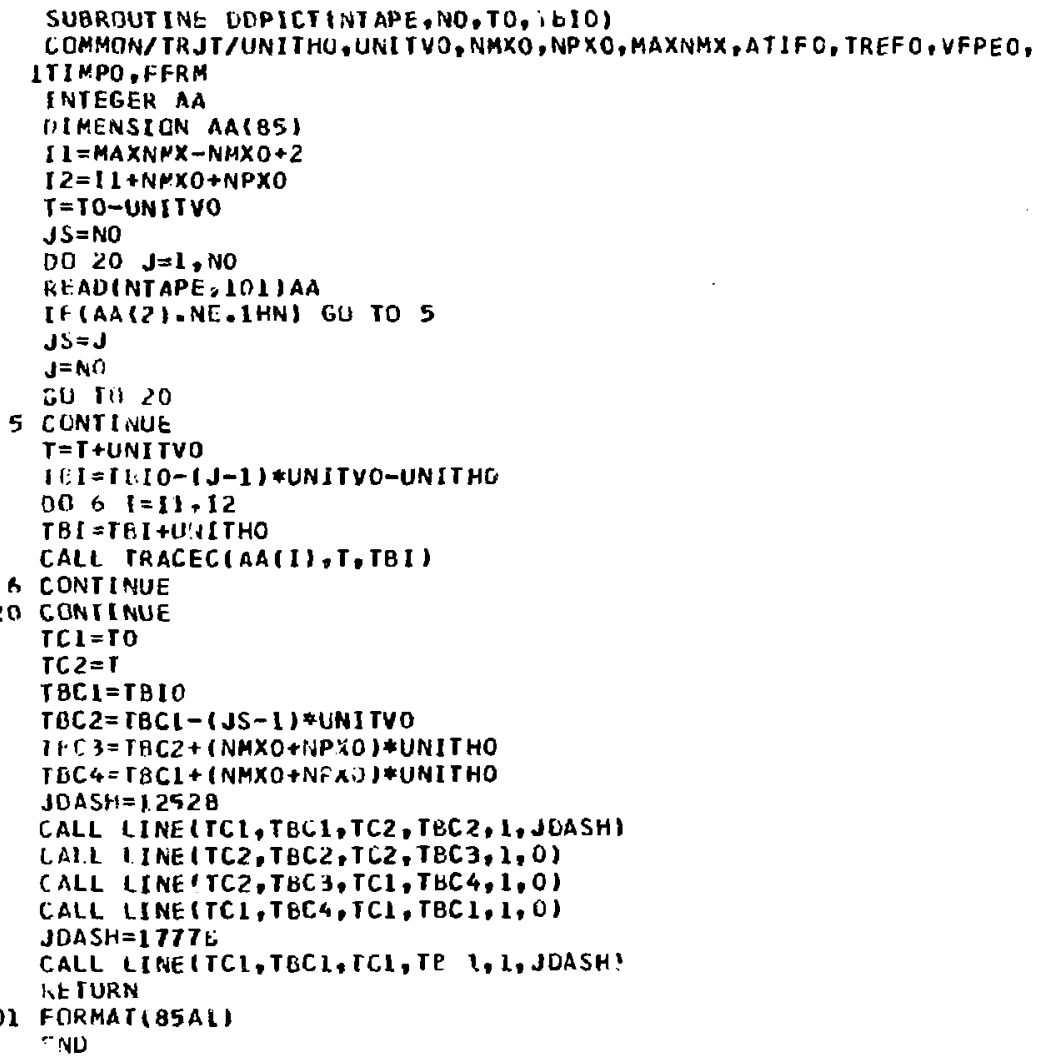




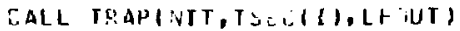

HII I I ) = r I ( 1$)$

16. COINT INIIE

$[: 13$ 3I $I=\{, 9 T$

HT ( I ) =hTT I I )

31 CLINITI HUE

C.ALL TICK(HTINT), I II)

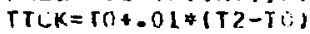

i) $20 \quad I=1$, NTCK

(i) $30 \quad J=1, N T M 1$

IFIILII).GT.HT(J)).(Ik. ILII).LT.HT(J+1)I) G0 ro 30

$t=(H T(J)-Z(I)) /(H T(J)-H T(J+1))$

$T E=T I M O-T S E C(J)-F *([S E C(J+1)-I S E C(J))$

CALL LINFITO,TB,TZ,T(1,1,0)

$L P=L(1)$

CALL EFORM(LP,ZN, S)

TPRNi $=$ TO- (.9*FFRM+(T2-TO))/(1-FFRM)

CALL SETLCHITPRNT, TL, 1,0,0,0,0)

WKI TE (100,TOL)LP, ZN,N

$J=N T M I$

30 CONTI INUE

20 CDNTINUE

TLFTM=TO- (FFRM(12-IO) $1 / 11-F F R M)$

TTCK $=$ TLFTM+.0L* T T2-TOI

CALL LINE(TLFTN,TBI3,TLFTM,TBIO,1,0)

CALL TICK (TBI3,TBIO)

L. $40 \quad I=1$, NTCK

CALL LINE(TLFTN,Z(I),TICK,Z(I), I, O)

40 CUNTI UUE

$Z P=2 \mid 1)$

CALL EFCRM ( $\angle F, Z N, N)$

THPRNT $=$ TB I 3-.025*(TEIO-TEI 3)

$T P R N T=T O-(1.1 * F F R N *(T 2-T O) / / 1-F F K M)$

CALL SETLCHITPRNT, TBPRNT, $1,0,0,0,1)$

WRI TE (1OO, POI) $\mathrm{CP}, 2 \mathrm{~N}, \mathrm{~N}$

$Z P=L(2)-2(1)$

CALL EF $C X M(L P, Z N, N)$

WRITE IIOC, 70IILP, LN, N

CALL LINE ITO,TBI3,T2,TB I3, 1, O)

CALL IICK $(T O, T 2)$

THTCK $=$ I $[13+.01 *(1$ E $10-T B I 3)$

J) $50 \quad I=1$, NTC.K

CALL LINE $(Z(I), T$ BS $\{3, Z(1), T B T C K, 1,0)$

$Z P=Z(【)$

CALL $\operatorname{RERM}(Z P, Z N, N)$

TBPRNT $=$ TUI3-1. ก\#FFRNF(TBI0-TBI3))/(1-FFRMI

CALL SFITLHIZ(I), TBPINT, $1,0,0,1,0)$

WRITE IIOO, 7OLIZP, ZN,N

50 COIUTI NUE

SETUR'N

70I FORMAT (F6, 3,AL, IL)

END!

SUPROUTINE EDPICTI.INTAPEI

INTEG:R AA

[)IMENSIUN AALES

$i=43$.

2 CONTINUE

RFAU (NTAFE, IOI) AA

IF(EUF, NTAPE) 99,1

1 CONTI INUE

If |AA(2).tG.IHA) FU TU 99 
IF(AAII).EL.1HI) Ex43.

$B=B-L$.

IF IB. GE.0.1 GO TO 3

CALL FRAME (1)

$B=42$.

3 CONTINUE

(ALL SETCH $(1, \ldots, 1,0,1,0,0)$

WRIIT (100, 10L)AA

;O TO ?

99 CONTI INUE

RETIJRN

IOL IUKMAT ( B5AL)

END

SUBROUT I INE TICK $(\times 1, \times 2)$

COMMON/TCK/ $2(100)$, NTCK

5 IGNS $=\times 1 * \times 2$

$A=\times 2-\times 1$

$A L=A L Q G L O(A)$

$N=A L$

IFIAL,LT.0.) N=N-1

$C=A /(10 . * * N)$

$\mathrm{NC}=\mathrm{C}$

IF (NC.GT.5) NC=5

IF (NC.LT.L) NC=1

$T S=N C *(10 . * *(N-1))$

IFISIGNS.LT.O.' GO TU 7

$N \times 1=0$

IF(XI.NE.0.) NXI=ALOGIO(ABSF(X) )

$E=X 1 /(10 . * * N \times 1)$

$\mathrm{NE}=\mathrm{E}$

$I F(X)$. LT. O. .) NE=NE-1

$E=N E$

$E=E *(10 . * * N \times 1)-T S$

$I=0$

5 CONTINUE

$E=E+T S$

IFIE.LT.XI) GO TO 5

IF(E.GT $\times 2)$ GO TOS 6

$I=I+1$

$Z I I I=E$

$60 \mathrm{TO} 5$

6 CONTINUE

NTCK $=1$

RETURN

7 CONTINUE

NTSL $=-X 1 / T S$

DO $15 \mathrm{~J}=1$, NTS!

$Z(J)=-(\operatorname{NTS} l+l-J) * T S$

15 CONTI NUE

I $\approx$ NTS I

$E=-T S$

8 CONTINUE

$E=E+T S$

IF(E.GT.X2) GO TO 6

$I=I+1$

I (I) $=\mathrm{E}$

GO TO $B$

END 


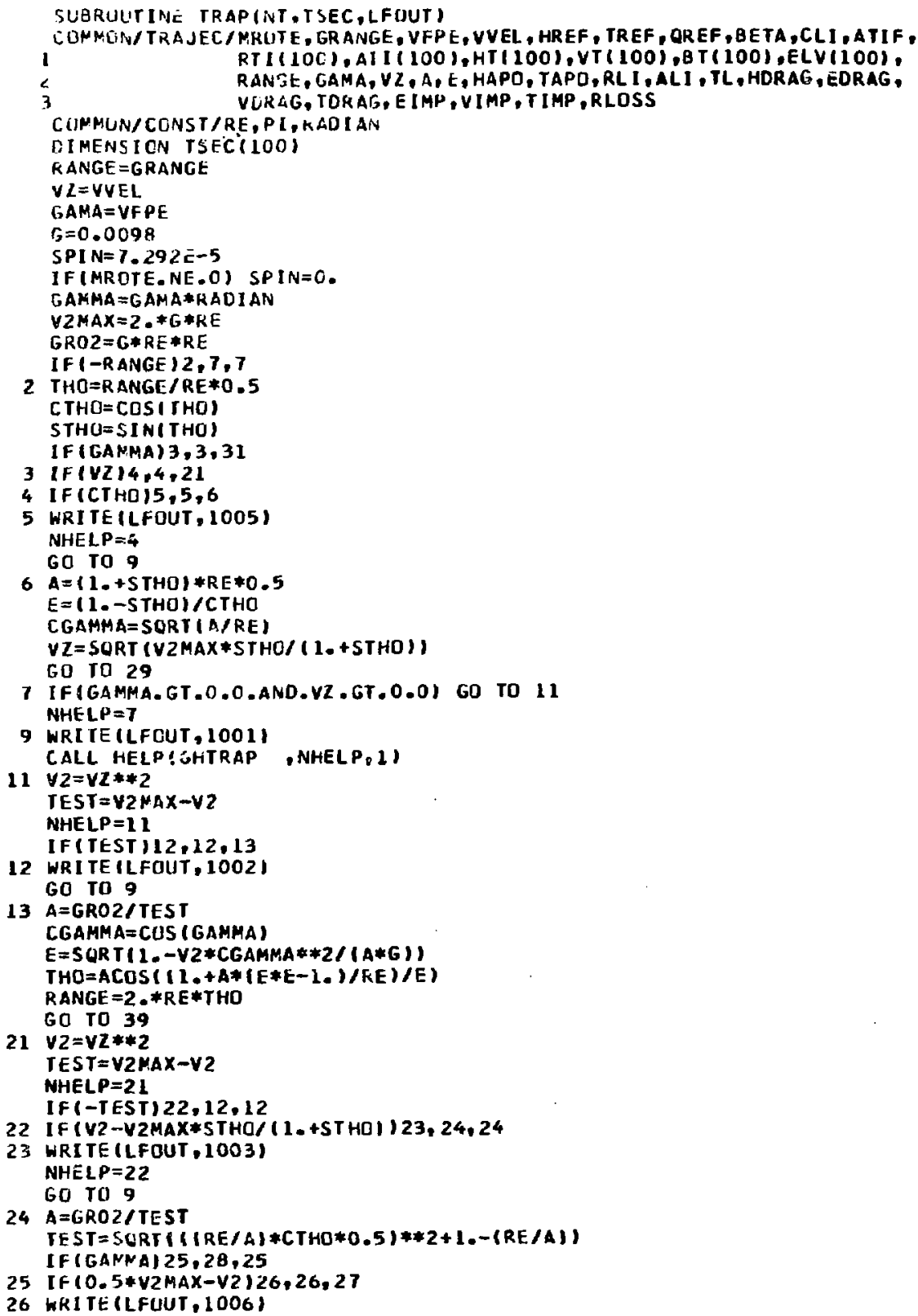

3 IF $(\cup Z) 4,4,21$

4 IF (CTHD) $5,5,6$

5 WRITE (LFOUT, 1005 ) NHELP $=4$ GO TO 9

$6 A=\{1 .+S T H O) * R E * 0.5$

$E=(1 .-S T H O) / C T H O$

CGAMHA $=$ SQR $(A / R E)$

$\checkmark Z=$ SORT (VZMAX *STHO/ $(1,+S T H O))$

GO TO 29

7 IFIGAMMA.GT.O.O.AND.VZ.GT.0.0) GO TO 11 NHELP $=7$

9 WRITE ILFOUT, 1001 I

CALL HELP!OHTRAP, NHELPDII

$11 \vee Z=V Z * 2$

TEST $=Y 2 H A X-V 2$

NHELP $=11$

IF (TEST ) 12,12,13

12 WRI TE ILFOUT, 1002 I

GO TO 9

$13 A=G R O 2 / T E S T$

CGAMMA $=$ CUS (GAMMA)

$E=S Q R T(1-V 2 * C G A M M A * * 2(\mid A * G))$

$T H O=A C O S(1,+A *(E * E-1,) / K E) / E)$

RANGE $=2$ ॰ $* R E * T H O$

GQ TO 39

$21 \vee Z=V Z * * 2$

TEST $=V 2 M A X-V 2$

NHELP=21

IF I-TEST I 22, 12,12

22 If I V2-V2MAX *STHO/ (1,+STHDI) 23, 24, 24

23 WRITE (LFOUT, 1003)

NHELP $=22$

60 TO 9

$24 A=G R O 2 / T E S T$

IEST $=$ SQRI $(\{\mid R E / A\} * C T H O * 0.5) * 2+1 .-(R E / A)\}$

IF ( GANNA) $25,28,25$

25 If $10.5 * V 2 M A X-V 2126,26,27$

26 WKITE (LFUUT, 1006$)$ 


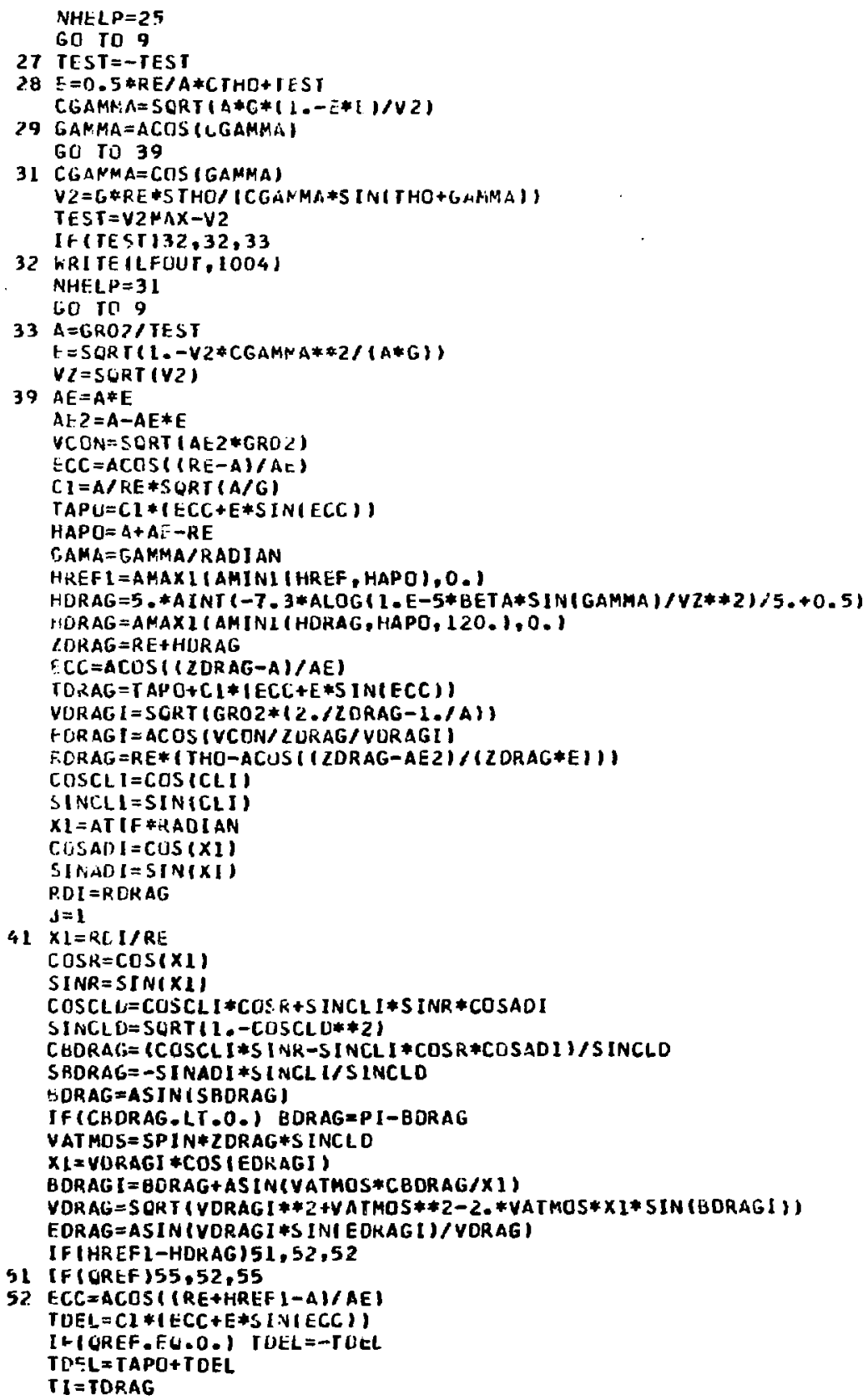


I! I = HORAG

$V I=V I: F \cdot A G$

$F I=$ EUKAG

$R I=R \Gamma I$

Cit. TOT 56

55 TDEL $=1 . E 10$

CALL KVOZAG $(G$, EETA, TLRAG,HDRAG, VDKAG, EDRAG,RDI, TDEL, HREF I, VI, EI, I ( I )

$T I=T i 3 t L$

$H I=H F E F I$

$56 T L=T R E F-T U E L$

$T I=I I+T L$

TIMP=1 E E1O

$H F=0.0$

CALL FVDRAG (G, BEIA, II,HI,VI,EI, RI, TIMP, HF, VIMP, EINP,RLOSS)

IF (0.0D1-ABS (RLLSS) $57,58,58$

57 ROI $=\$ 1 I I-R L C S S$

$\mathfrak{J}=\mathbf{J}+1$

IF(J.TT.1O) CALL FELP(GHTRAP ,57,1)

60 TO 41

58 TAPO=TAPO+TL

TORAG $=$ TORAG+TL

RLOSS = RCRAG-RDI

CALL C.UORDX $(0,1, K D I$, ATIF, HDKAG,XI,X2,X3,XDI, YDI, ZDI,X4,X5)

LALL ASPECTICOSCLI,SINCLI, $0, \ldots X U, Y C I, 20 I, 1,0,0,0,, X I D, Y I D, 2 I D I$

A TD $=(E$ U URAGI I I ) /KAUI AN

$T P=T L$

$I=0$

IF (N) $60,95,60$

6) $I=I+I$

I I = TS[C I I )

$t \perp E M=(T I-T A P[]) / C 1$

IF \{ T I -TL I $62,62,63$

6) $\quad Z I=R i$

$H I=0.6$

$\mathbf{V} !=\mathrm{V} Z$

$E I=G A A_{N A N}$

RI=RA :JGE

60 TO 68

63 IF(TI-TUAAG)64,64,80

$64 \mathrm{ECC}=\mathrm{tM}$

$65 \quad$ CCCI $=E C C$

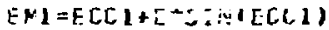

1. $C C=E C, C 1+(E M-E M) /(1++E * C O S(E C(1))$

IF (AHS (ECC-ECCI)-1.E-6*ABS (ECC) $160,66,65$

Gt. $7.1=A \div(1 .+E \neq C O S(E C C)$,

$\| I=Z I-R E$

$V I=56 R T(C R \cap 2 *(2 . / 7 I-I . / A))$

$E I=A C, L S(V C O N / Z I / V I)$

$R I=R t *(T H A-S I G N(A C O S(\{Z I-A E 2) /(2 I * E)), E M))$

68 F $T O=R I-H C K A G$

[. ALL COOFUXIHURAU, 1, RTO,ATD,HI,X1,X2,X3,XTD,YTD, 2 TO, X4,X5)

CALL ASPECTICDSCLL, S INCLO, HURAG,XIO,YIO,ZID, I,XTD, YTO, ZTO,XII, YTI,

LCTI

CLLNG $=S+[N *(T D R A G-A N A X I(T I, T L))$

$Y 1=A S I N(S I N C L I * S I N(0.5 * 0 L O N G)$

$A I I=-A S I N(C L S)(0.5 * D L U N G) / C O S(X)) / R A D I A N$

IF (CLSCLI.LI.0.) AIII=180.-AII

$R 11=2$ * RF $\# 1$

CALL CCCRIXXI0, I, KII,AII, 0, XI,X2,X3,XII,YII,ZII,X4,X5I

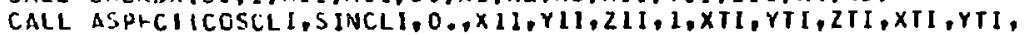
12 I I )

CALL LRC:UXX(0, 2, XTI,YTI,ZTI,RI, AI, XI, X2, X3, X4, X5, X6) 
I FITL-TII71,71.67

$71 \operatorname{COSA}=-\operatorname{CSS}(\mathrm{BDRAG} I)$

$Y_{1}=R T D / R E$

$\operatorname{COSR}=\operatorname{COS}(\times 1)$

$S I N R=S I N(X I)$

COSCLT $=C O S C L D * C D S K+S 1 N C L D * S I N R * \operatorname{COSA}$

SI NCLT $=$ SORT $(1,-\operatorname{COSCL} T * 2)$

COSG $=(C O S C L O * S I I N R-S$ INCLD*COSR *CUSA)/SINCLT

$S I N B=5 I N C L D \# S I N$ IBCRAGI I/SINCLT

VATMOS=SPIN*ZI*SINCLT

$X 1=V I * C C S\{E I\}$

$V T I=S G R T(Y I * 2+V A T M O S * * 2-2$ * $V A T M O S * X I * S I N B)$

ETI =ASIN(YI*SIN(EI)/VTI)

X2 =VTI*COS (ETI)

SINBTI $=(X 1 * 5$ INB-VATMOS $) / \times 2$

Cos $\theta$ T $1=X 1 * \cos B / \times 2$

GO TO 91

60 IF (TI T TMP) 81,81,86

$81 \mathrm{HF}=0.0$

IF (TP-TDRAG) 82,82,B3

$82 T P=$ TORAG

$H I=H U R A G$

$\checkmark T I=V U R A G$

$E T I=E D R A G$

$R I=R O I$

83 CALL RYGRAG IG, BETA,TP,HI, YTI, ETI,RI, TI,HF, YTI,ETI,RII

$H I=H F$

$T P=T$ I

$A !=A T$ If

$X I=R I / R E$

$\operatorname{COS} R=\operatorname{Cos}(x))$

$S I N R=S I N(X 1)$

COSCLT $=$ COSCLI $* C O S R+S I N C L I * S I N R * C O S A D I$

SINCLT $=$ SORT $(1,-\operatorname{COSCLT} * 2)$

CCSBTI $=$ CCOSCLI $* S$ LNR-SINCL I $*$ COSR*COSAOII/SINCLT

S I NBT I =-S I NADI *S I NCL I/S INCL T

GD TO 9

$6 \quad R I \approx 0.0$

$A I=0,0$

$H I=0.0$

$67 \vee T I=0.0$

SINBT $I=0.0$

COSBTI $I=1.0$

E $I I=U .0$

91 I F I I -NT $192,92,96$

$92 \mathrm{KTI}(1)=\mathrm{AI}$

$A T I I I)=A I$

HT (I) $=H I$

$V T\{\}=V T I$

I TI I ) =ASI NISINBTI /RAD I AN

IF (C.USATL.LT .0.) \&T III = 180.-BT (I)

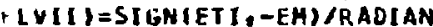

I F I T I - I I MP $\cdots 3,94,94$

9.3IFII-NTI60 $\approx 5,60$

$94 d T=1$

$95 \mathrm{I}=\mathrm{NI}+\mathrm{l}$

$I I=T C$

tod $\mathrm{ru} 6 \mathrm{t}$

96. $L I=E I$

$A\lfloor I=A I$

F DRAG $=-E D R A G / R A O I A N$

I $M P=-E$ I MP / RADIAN

RETURN 
LOOI FCRMAT (28HOTRAJECTORY DATA INPUT IRROR)

2002 FORMAT (40HOLAUNCH VELOCITY EXCLEDS ESCAPE VELOCITY)

1003 FURMAT (5OHOLAUNCH VELOCITY LESS THAN MINIMUM-ENERGY VELOCITY)

1004 FURMAT (27HOLAUNCM ELEVATION TOO LARGE)

1005 FDRMATIB9HOMINIMUR-LNERGY TRAJECTURY REQUESTED FOR SURFACE RANGE E IXCEEUING HALF EARIH CIRCUMFEREIHCE I

1006 FORMAT (3QHODEPRESSED TRAJECTORY INTERSECTS EARTH) END

SUBRUUT INE COORDX (HO, MODE, RXSE, AYAA,HZEH, R,A,H,X,Y,Z,S,E)

CDMMON /CONST/RE, PI, RADIAN

$R E H=R E+H O$

GO TO $(11,21,31,41), M O D E$

$11 \mathrm{R}=\mathrm{R} \times S \mathrm{SE}$

$A=A$ YAA

$H=H Z E H$

UO=R/RE

$J 2=A * R A D I A N$

$U 3=R E+H$

$U I=U 3 * S I N(U 0)$

$X=U L * S I N(U 2)$

$Y=U 1 * \cos \left(U_{2}\right)$

$Z=U 3 * \cos (U 0)-R E H$

IFIUO.E. .0.$) \quad Z=H-H O$

I F IUL $123,25,23$

$21 X=R X S E$

$Y=A Y A A$

$Z=H Z E H$

$U I=X * 2+\gamma * \pm 2$

I $F$ (U1) 22, 24,22

$22 U 2=R E H+Z$

$U 3=S G R T(U 1+U Z * 2)$

$U 1=S G A T$ (UI)

$R=A S I N(U 1 / U 3) * R E$

IF (U2. LT - 0.0) $R=P$ I*RE-R

$A=A S I N(X / U L) / R A D I A N$

IF (Y.LT.0.0) $A=180 .-A$

$H=U 3-R E$

$23 S=S O R T(U 1 * * 2+Z * * 2)$

$E=A S I N(Z / S) / R A D I A N$

GO TO 99

$24 \mathrm{R}=0$.

$A=0$.

$7+2$

$25 S=, J S(Z)$

$F=90$.

IFIZ.LT.O.OUI E $=-90$.

GO TO 99

$31 S=R \times S E$

$A=A Y A A$

$F=H Z E H$

U2 $=A * R A B I A N$

$U 3=E *$ RADI AN

$U l=S * \operatorname{CUS}\left(U_{3}\right)$

$X=U(* S I N(U 2)$

$Y=U 1 * \operatorname{COS}\left(\mathrm{L}^{2}\right)$

$Z=S * \operatorname{Sin}(U 3)$

$U 2=R E H+Z$

$U 3=\operatorname{SQRT}(U 1 * * 2+U 2 * * 2)$

$R=A S I N(U I / U 3) * R E$

IF (U2 - LT_. 0.0) R=PI*RE-R

$\mathrm{H}=\mathrm{U} \simeq-\mathrm{RE}$ 
$60 \quad 1099$

$41 \mathrm{E}=\mathrm{R} \times \mathrm{SE}$

$A=A Y A A$

$H=H 2 T^{-} H$

$U E=A * K$ ADI AN

$U 3=E * A, A C I A W$

$\operatorname{CnSE}=$ CI'S(U3)

$S$ INE $=S I N(U 3)$

$S=H T O S(H Q, H, C O S E, S I N E)$

II $=S * \operatorname{COSE}$

$X=U 1 \neq S I N(U 2)$

$Y=U 1 * \cos (U 2)$

$\angle \approx S * S$ INE

U3 $=$ RE $+H$

$R=\triangle S I N(U 1 / U 3) * R E$

If (REHI+Z.LT.0.0) $R=P I * R E-R$

99 RETURH

C. NO

SUBRUUTINE HELP (SUBNAM, NST ATE, KOPT)

DIKEVSION HOLRTH( 2 )

UASA HOLRTH/3H THNON/

WGI TE (59, LOIHOLRTHIKOPT), SUBNAM, NSTATE

IFIKOPT.EQ.II CALL EXIT

RETURN

I0 FORMATIIHOA3,37HFATAL ERHOR DISCOVEIED IN SUHRDUTIIE AG,2OH AT STA ITENEA: NUMBERI6)

END

SURROUT INE RVDRAG (GO,BETA, TI,HI,VI,EI,RI, TF,HF, VF,EF,RF)

COMMON /CONST/RE,PI, 'RAOLAN

UELTAH $=0.5$

$T=\mathbf{T} \mathbf{I}$

$H=H I$

$V=\mathbf{V I}$

$E=E I$

$Q=R$ I

11 DELTAT $=$ TF-T

SINE $=$ : INIE I

DH

I F $(-D H) 12,21,21$

12 HBAR= $1-D H / 2$.

$G B A K=60 *(R E /(R E+H B A R)) * * 2$

CALL ATMISDI0, HBAR, RHDEAR, DUM1, DUM2, DUM3, DUM4, DUM5, DUMEI

$B=47$. $R B 1-9$ *RVBETA (UETA, HBAR, RHUBAR, V,E)

$\checkmark 2 L Q=2 . * B * 5$ INE/RHCBAR

$D V=G P^{\prime} A R *(I * I V-V / V, E L I) * D H$

If IUVI $13,15,15$

13 I $F((V+D V) * A B S(V+U V)-V 2 E G) 14,15,15$

14 DV $=$ SQRT (VLEG) -V

$15 V B A R=V+D V / 2$.

COSE $=\operatorname{COS}(E)$

IJE $=$ GU AR *COSE/S INE/VBAR/VBAR *OH

I $A A R=A F I N I(E+D E / 2, \ldots P I / 2$.)

SE $U A R=S$ (N (EBAR)

CEAAR =CLS (EBAR)

L. TNE = CE $H A R / S E B A R$

$I+H=O H * C T I N E$

$X=R E+H-D H$

$D Y=O H I+D R * D R / 2$. $/ X$

$U R=D Y * C T N E / X$ 


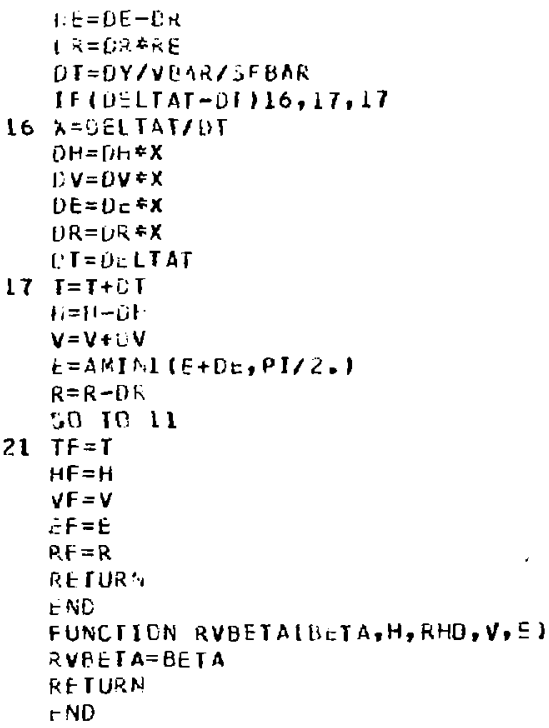

SUHKLUT IPE ASPECT ICUSCLO,SINCLU,HO,X1,Y1,Z1, NPOINT,XZ,Y2,Z2,XEI, (Y2L,Z21)

DI MENSICN $\times 2(2), Y Z(2), Z 2(2), \times 21(2), Y 21(2), 221(2)$

CONMON/CONST/RE, PI, RAOIAN

$\mathrm{REH}=\mathrm{RE}+\mathrm{HU}$

UI $=X I \neq \neq 2+Y I \neq \div 2$

[F(UI) $L, 2,1$

$1 \quad U 2=\{E H+Z I$

U13 $=50 R T(U 1+U 2 * \div 2)$

$U L=S C K T(U 1)$

SINZI $=U 1 / 43$

CUSA1 $=U 2 / U 3$

$S I N A I=\times 1 / U 1$

COSAI $=$ Y $1 / U]$.

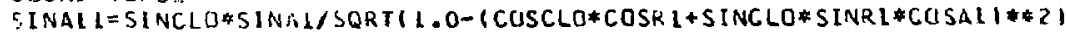
IFISINAII.GT. I.O) SINAII=1.0

IFISINAII, LT. $(-1,0)\}$ SINAI $1=-1.0$

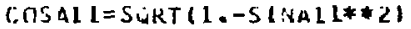

IF ISINCLI]*COSRI*CUSA1-COSCLO*SINRI.LT. 0.0$) \operatorname{COSA} 11=-\operatorname{COSAII}$

¿U TU 3

$2 \quad 33=R E H+L 1$

3 DU $9 \mathrm{~N}=1$, NPUINT

$X=X 2(N)-X 1$

$Y=Y Z(N)-Y 1$

$l=Z 2(N)-\angle I$

IFIU1) $4,5,4$

$4 Y Y Y=X *$ SINAI +Y*COSAI

$X X=X * C$ OSAI - Y*SIINA 1

$Y Y=Y Y Y * C U S R I-L * S I N R I$

$X=X X * C D S A 11+Y Y * S I N A 1 I$

$Y=Y Y * C O S A I 1-X X \pitchfork S I N A I I$

$Z=Y Y Y * S 1 N R 1+Z$ CUSR 1

$5 \times 21(N)=x$ 
$Y 21(N)=Y$

Z? $|\backslash N|=Z$

9 CLNTISUE

RETURN

FNO

FUNCTION HT IS (HI, HZ, COSE, S INE)

COMMON /CONST/RE,PI, KAOIAN

$R I=R E+H I$

$R 2=R i+H 2$

$S I N U=R I * C U S E / R 2$

CUSO = SCRT $(1 .-S I N C * * 2)$

HTOS $=R 2 * C O S D-R 1 * S$ INE

RETURN

FNU 


\section{SUBROUTINE ATMOSD}

This subroutine is listed in Ref. 6. 